\title{
Total synthesis of borrelidin
}

Tohru Nagamitsu, ${ }^{1}$ Daisuke Takano, ${ }^{1}$ Kaori Marumoto, ${ }^{1}$ Takeo Fukuda, ${ }^{1}$ Kentaro Furuya, ${ }^{1}$ Kazuhiko Otoguro, ${ }^{2}$ Kazuyoshi Takeda,${ }^{3}$ Isao Kuwajima, ${ }^{4}$ Yoshihiro Harigaya, ${ }^{1}$ and Satoshi_mura*2,4

${ }^{1}$ School of Pharmacy, Kitasato University, 5-9-1 Shirokane, Minato-ku, Tokyo 1088641, Japan

${ }^{2}$ The Kitasato Institute, 5-9-1 Shirokane, Minato-ku, Tokyo 108-8642, Japan

${ }^{3}$ Department of Medical Chemistry, Yokohama College of Pharmacy, 601 Matanocyo, Hodogayaku, Yokohama-shi, Kanagawa 245-0066, Japan

${ }^{4}$ Kitasato Institute for Life Sciences and Graduate School of Infection Control Sciences,

Kitasato University, 5-9-1 Shirokane, Minato-ku, Tokyo 108-8641, Japan

Table of contents

1. General Information

2. Experimental Procedures and Characterization Data

Compound 2'

Compound $\mathbf{3}$

Compound 4

Compound 6a

S4

Compound $\mathbf{6 b}$

Compound 7

Compound 8

S6

Compound 11

S7

Compound 12

S8

Compound 13

S8

Compound 14

S9

Compound 14a

S10

Compound 14b

S11

Compound 15

S11

Compound 16

S12

Compound 19

S14

Compound 21

S15

Compound 25

S16

Compound 26

S16

Compound 27

Compound 28

S18

Compound 29a

S29

Compound 29b

S30

Compound 30

S40

Compound 30a

Compound $\mathbf{3 1}$

Compound 31a

Compound 31b

Compound 38 (from 56, only experimental procedure) 
Compound 43

$\begin{array}{ll}\text { Compound } 44 & \mathrm{~S} 19\end{array}$

$\begin{array}{ll}\text { Compound } 45 & \mathrm{~S} 20\end{array}$

$\begin{array}{ll}\text { Compound } 46 & \text { S21 }\end{array}$

Compound $47 \quad$ S22

$\begin{array}{ll}\text { Compound } 48 & \text { S24 }\end{array}$

Compound $51 \quad$ S25

Compound $\mathbf{5 3} \quad$ S26

Compound 55

Compound $56 \quad$ S28

$\begin{array}{ll}\text { Compound } \mathbf{5 7} & \text { S39 }\end{array}$

$\begin{array}{ll}\text { Compound 58a } & \text { S37 }\end{array}$

$\begin{array}{ll}\text { Compound 58b } & \text { S38 }\end{array}$

$\begin{array}{ll}\text { Compound 59a } & \text { S30 }\end{array}$

Compound 59b $\quad$ S31

Compound 60a $\quad$ S32

Compound 60b $\quad$ S32

Compound 61a $\quad$ S33

Compound 61b $\quad$ S33

Compound 61b'

Compound 62a $\quad$ S34

Compound 62b $\quad$ S36

3. NMR spectra S'1 - S'91 
General Information. Commercial reagents were used without further purification unless otherwise indicated. Organic solvents were distilled and dried over molecular sieves $3 \AA$ or $4 \AA$. Reactions were performed in flame-dried glassware under positive Ar pressure with stirring by a magnetic stirbar unless otherwise indicated. Cold baths were generated as follows: $0{ }^{\circ} \mathrm{C}$, wet ice/water; $-78{ }^{\circ} \mathrm{C}$, dry ice/acetone. Flash chromatography was performed on silica gel $60 \mathrm{~N}$ (sherical, neutral, particle size 40-50 $\mu \mathrm{m})$. TLC was performed on $0.25 \mathrm{~mm}$ E. Merck silica gel $60 \mathrm{~F} 254$ plates and visualized by UV $(254 \mathrm{~nm})$ and cerium ammonium molybdenate.

(2S,4R)-5-Hydroxy-2,4-dimethylpentyl acetate (6)

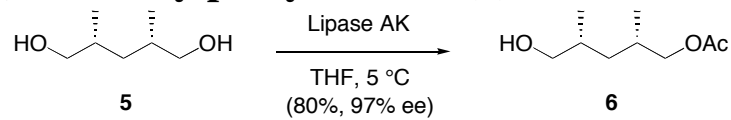

This compound was prepared according to Mori's procedure. (Fujita, K.; Mori, K. Eur. J. Org. Chem. 2001, 66, 493-502.)

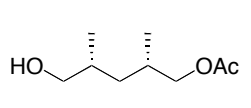

6

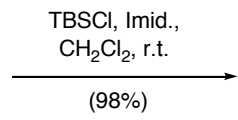

TBSO

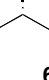

$6 a$

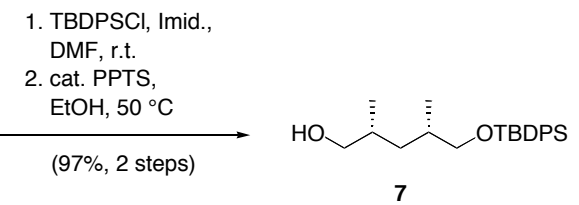

(2S,4R)-5-(tert-butyldimethylsilyloxy)-2,4-dimethylpentyl acetate (6a)

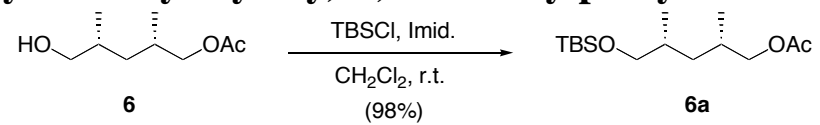

To a solution of $6(1.01 \mathrm{~g}, 5.83 \mathrm{mmol})$ in $\mathrm{CH}_{2} \mathrm{Cl}_{2}(20 \mathrm{ml})$ were added imidazole $(790$ $\mathrm{mg}, 11.6 \mathrm{mmol})$ and $\mathrm{TBSCl}(1.30 \mathrm{~g}, 8.74 \mathrm{mmol})$ at room temperature. The resulting solution was stirred for $30 \mathrm{~min}$ and diluted with water. The aqueous phase was extracted with $\mathrm{CH}_{2} \mathrm{Cl}_{2}$. The combined organic extracts were dried over anhydrous $\mathrm{Na}_{2} \mathrm{SO}_{4}$ and concentrated in vacuo. Flash chromatography (30:1 hexanes/EtOAc) afforded 6a (1.64 $\mathrm{g}, 98 \%)$ as a colorless oil. $[\alpha]_{\mathrm{D}}^{22}+5.6^{\circ}\left(c \mathrm{0.24}, \mathrm{CHCl}_{3}\right)$; IR $(\mathrm{KBr}) 2956,2931,2892$, 2888, 1743, 1251, $1238 \mathrm{~cm}^{-1}$; ${ }^{1} \mathrm{HNMR}\left(270 \mathrm{MHz} \mathrm{CDCl}_{3}\right) \delta 0.01(\mathrm{~s}, 6 \mathrm{H}), 0.87(\mathrm{~s}, 9 \mathrm{H})$, $0.88(\mathrm{~d}, 3 \mathrm{H}, J=6.6 \mathrm{~Hz}), 0.90(\mathrm{~m}, 1 \mathrm{H}), 0.92(\mathrm{~d}, 3 \mathrm{H}, J=6.6 \mathrm{~Hz}), 1.44(\mathrm{~m}, 1 \mathrm{H}), 1.67(\mathrm{~m}$, $1 \mathrm{H}), 1.88(\mathrm{~m}, 1 \mathrm{H}), 2.02(\mathrm{~s}, 3 \mathrm{H}), 3.34(\mathrm{dd}, 1 \mathrm{H}, J=9.6,6.3 \mathrm{~Hz}), 3.41(\mathrm{dd}, 1 \mathrm{H}, J=9.6,5.6$ $\mathrm{Hz}), 3.80(\mathrm{dd}, 1 \mathrm{H}, J=10.9,6.9 \mathrm{~Hz}), 3.94(\mathrm{dd}, 1 \mathrm{H}, J=10.9,5.3 \mathrm{~Hz}) ;{ }^{13} \mathrm{C}-\mathrm{NMR}(67.5$ $\left.\mathrm{MHz}, \mathrm{CDCl}_{3}\right) \delta-5.4,17.4,17.8,18.2,20.9,25.9,30.0,33.0,37.4,68.0,69.3,171.2$; HRMS (FAB, $m$-NBA) $[\mathrm{M}+\mathrm{H}]^{+}$calcd for $\mathrm{C}_{15} \mathrm{H}_{33} \mathrm{O}_{3} \mathrm{Si} 289.2199$, found 289.2193.

(2S,4R)-5-(tert-Butyldimethylsilyloxy)-2,4-dimethylpentan-1-ol (6b) 


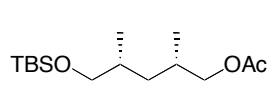

$6 a$

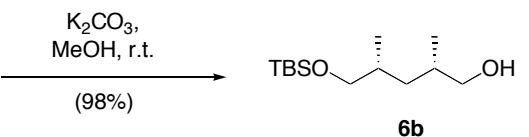

$6 b$

To a stirred solution of $\mathbf{6 a}(1.64 \mathrm{~g}, 5.72 \mathrm{mmol})$ in $\mathrm{MeOH}(6 \mathrm{ml})$ was added potassium carbonate $(870 \mathrm{mg}, 6.29 \mathrm{mmol})$. The reaction was stirred at room temperature for $2 \mathrm{~h}$, and then diluted with water. The aqueous phase was extracted with EtOAc. The combined organic extracts were dried over anhydrous $\mathrm{Na}_{2} \mathrm{SO}_{4}$ and concentrated in vacuo. Flash chromatography (10:1 hexanes/EtOAc) afforded $\mathbf{6 b}(1.47 \mathrm{~g}, 98 \%)$ as a colorless oil. $[\alpha]_{\mathrm{D}}^{22}+0.8^{\circ}\left(c 0.32, \mathrm{CHCl}_{3}\right)$; IR (KBr) 3411, 2956, 2929, 2858, 1471, 1255, 1093, $837 \mathrm{~cm}^{-1}$; ${ }^{1} \mathrm{H}-\mathrm{NMR}\left(270 \mathrm{MHz}, \mathrm{CDCl}_{3}\right) \delta 0.03(\mathrm{~s}, 6 \mathrm{H}), 0.88(\mathrm{~m}, 1 \mathrm{H}), 0.89$ $(\mathrm{s}, 9 \mathrm{H}), 0.90(\mathrm{~d}, 3 \mathrm{H}, J=6.6 \mathrm{~Hz}), 0.93(\mathrm{~d}, 3 \mathrm{H}, J=6.6 \mathrm{~Hz}), 1.42(\mathrm{~m}, 1 \mathrm{H}), 1.70(\mathrm{~m}, 1 \mathrm{H})$, $1.81(\mathrm{~m}, 1 \mathrm{H}), 3.32-3.52(\mathrm{~m}, 4 \mathrm{H}) ;{ }^{13} \mathrm{C}-\mathrm{NMR}\left(67.5 \mathrm{MHz}, \mathrm{CDCl}_{3}\right) \delta-5.4,17.7,17.8,18.3$, $25.9,33.2,33.3,37.3,68.1,68.3$; HRMS (FAB, $m$-NBA) $[\mathrm{M}+\mathrm{H}]^{+}$calcd for $\mathrm{C}_{13} \mathrm{H}_{31} \mathrm{O}_{2} \mathrm{Si}$ 247.2093, found 247.2079.

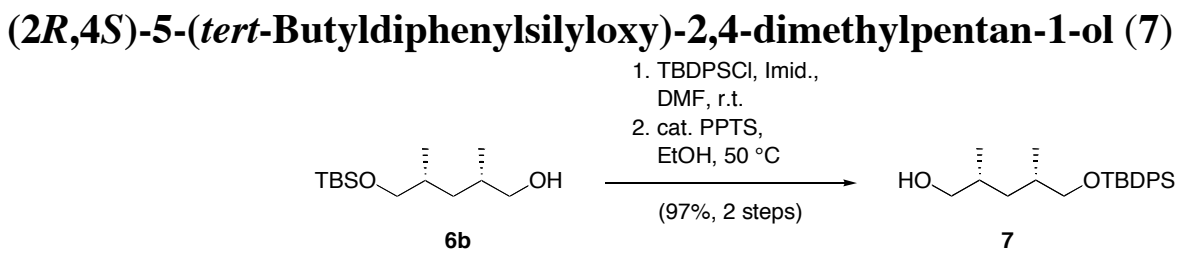

To a solution of $\mathbf{6 b}(3.10 \mathrm{~g}, 12.6 \mathrm{mmol})$ in DMF $(63 \mathrm{ml})$ were added imidazole $(1.70 \mathrm{~g}$, $25.2 \mathrm{mmol})$ and TBDPSCl $(4.90 \mathrm{ml}, 18.9 \mathrm{mmol})$ at $0{ }^{\circ} \mathrm{C}$. The reaction was allowed to warm to room temperature and stirred for $30 \mathrm{~min}$. After addition of water, the reaction mixture was extracted with EtOAc. The combined organic extracts were dried over anhydrous $\mathrm{Na}_{2} \mathrm{SO}_{4}$ and concentrated in vacuo. Flash chromatography (50:1 hexanes/EtOAc) afforded the corresponding disilyl ether (5.52 g) including unseparable impurities.

To a solution of the disilyl ether in EtOH (40 ml) was added PPTS (1.43 g, $5.70 \mathrm{mmol})$, and the mixture was allowed to warm up to $50{ }^{\circ} \mathrm{C}$. After $7 \mathrm{~h}$, water was added and the aqueous phase was extracted with EtOAc. The combined organic extracts were dried over anhydrous $\mathrm{Na}_{2} \mathrm{SO}_{4}$ and concentrated in vacuo. Flash chromatography (15:1 hexanes/EtOAc) afforded 7 (4.19 g, 97\% for 2 steps) as a colorless oil. $[\alpha]_{D}^{23}+1.8^{\circ}(c$ $\left.0.23, \mathrm{CHCl}_{3}\right)$; IR (KBr) 3455, 2931, 2856, 1112, 1091, $702 \mathrm{~cm}^{-1} ;{ }_{1} \mathrm{H}-\mathrm{NMR}(270 \mathrm{MHz}$, $\left.\mathrm{CDCl}_{3}\right) \delta 0.91(\mathrm{~d}, 3 \mathrm{H}, J=6.6 \mathrm{~Hz}), 0.95(\mathrm{~m}, 1 \mathrm{H}), 0.98(\mathrm{~d}, 3 \mathrm{H}, J=6.6 \mathrm{~Hz}), 1.08(\mathrm{~s}, 9 \mathrm{H})$, $1.49(\mathrm{~m}, 1 \mathrm{H}), 1.65(\mathrm{~m}, 1 \mathrm{H}), 1.76(\mathrm{~m}, 1 \mathrm{H}), 3.36(\mathrm{dd}, 1 \mathrm{H}, J=10.2,6.6 \mathrm{~Hz}), 3.45(\mathrm{~m}, 1 \mathrm{H})$, $3.51(\mathrm{dd}, 1 \mathrm{H}, J=9.6,5.3 \mathrm{~Hz}), 3.55(\mathrm{dd}, 1 \mathrm{H}, J=9.6,5.3 \mathrm{~Hz}), 7.35-7.72(\mathrm{~m}, 10 \mathrm{H}) ;{ }^{13} \mathrm{C}-$ $\operatorname{NMR}\left(67.5 \mathrm{MHz}, \mathrm{CDCl}_{3}\right) \delta 17.4,17.9,19.3,26.9,33.1,37.1,68.2,68.7,127.6,129.5$, 134.0, 135.6; HRMS (FAB, $m$-NBA) $[\mathrm{M}+\mathrm{H}]^{+}$calcd for $\mathrm{C}_{23} \mathrm{H}_{35} \mathrm{O}_{2} \mathrm{Si} 371.2406$, found 371.2415 .

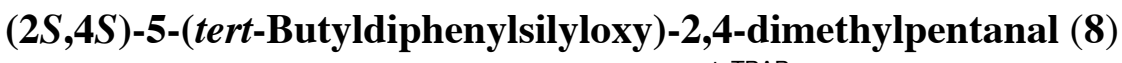

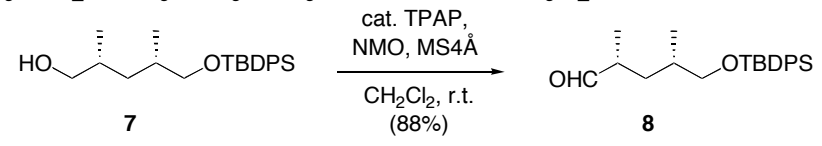

To a solution of 7 (3.13 g, $8.44 \mathrm{mmol})$ in $\mathrm{CH}_{2} \mathrm{Cl}_{2}(88 \mathrm{ml})$ were added dried MS4 $\mathrm{A}$ (17.5 g), NMO (1.09 g, $9.28 \mathrm{mmol})$ and TPAP $(147 \mathrm{mg}, 420 \mu \mathrm{mol})$ at room temperature. The 
reaction mixture was stirred for $30 \mathrm{~min}$ and filtered through a silica pad. After evaporation of the filtrate, the residue was purified by flash chromatography $(50: 1$ hexanes/EtOAc) to afford $8(2.73 \mathrm{mg}, 88 \%)$ as a colorless oil. $[\alpha]_{\mathrm{D}}^{23}-6.3^{\circ}(c 0.40$, $\mathrm{CHCl}_{3}$ ); IR (KBr) 2960, 2931, 2858, 1727, 1427, 1112, $1089 \mathrm{~cm}^{-1} ;{ }^{1} \mathrm{HNMR}(270 \mathrm{MHz}$, $\left.\mathrm{CDCl}_{3}\right) \delta 0.94(\mathrm{~d}, 3 \mathrm{H}, J=6.6 \mathrm{~Hz}), 1.04(\mathrm{~d}, 3 \mathrm{H}, J=6.6 \mathrm{~Hz}), 1.06(\mathrm{~s}, 9 \mathrm{H}), 1.14(\mathrm{~m}, 1 \mathrm{H})$, $1.75(\mathrm{~m}, 1 \mathrm{H}), 1.90(\mathrm{~m}, 1 \mathrm{H}), 2.39(\mathrm{~m}, 1 \mathrm{H}), 3.49(\mathrm{~d}, 2 \mathrm{H}, J=5.6 \mathrm{~Hz}), 7.34-7.70(\mathrm{~m}, 10 \mathrm{H})$, $9.54(\mathrm{~d}, 1 \mathrm{H}, J=2.3 \mathrm{~Hz}) ;{ }^{13} \mathrm{C}-\mathrm{NMR}\left(67.5 \mathrm{MHz}, \mathrm{CDCl}_{3}\right) \delta 14.1,17.2,19.3,26.9,33.3$, $34.5,44.1,68.4,127.6,129.6,133.7,135.6,205.2$; HRMS (FAB, $m$-NBA) $[\mathrm{M}]^{+}$calcd for $\mathrm{C}_{23} \mathrm{H}_{32} \mathrm{O}_{2} \mathrm{Si} 368.2171$, found 368.2142. 


\section{(S)-1-[(4,4-Dibromo-2-methylbut-3-enyloxy)methyl]-4-methoxybenzene (9)}

$$
\int_{9}^{\mathrm{Br}}
$$

This compound was prepared according to the known procedure. (Paquette, L. A.; Guevel, R.; Sakamoto, S.; Kim, I. H.; Crawford, J. J. Org. Chem. 2003, 68, 6096-6107.)

tert-Butyl[(2S,4R,8S)-9-(4-methoxybenzyloxy)-2,4,8-trimethylnon-6ynyloxy]diphenylsilane (11)

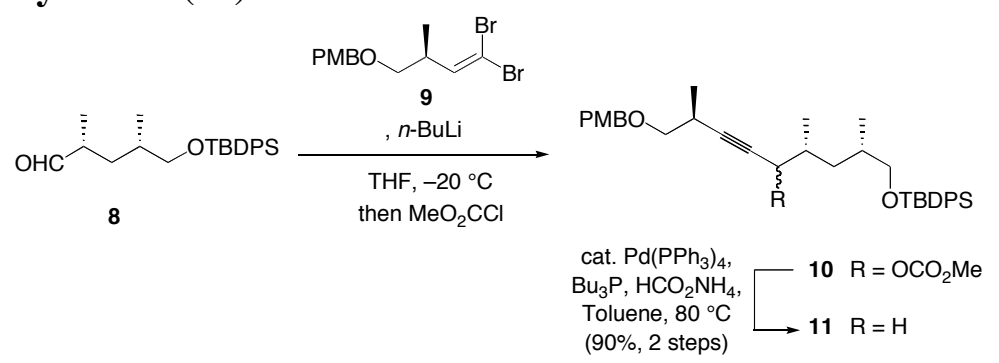

To a solution of 9 (2.66 g, $7.33 \mathrm{mmol})$ in THF $(22.4 \mathrm{ml})$ was added $n$-BuLi $(1.58 \mathrm{M}$ solution in hexane, $9.28 \mathrm{ml}, 14.7 \mathrm{mmol}$ ) at $-78{ }^{\circ} \mathrm{C}$, and the mixture was allowed to warm up to $-40^{\circ} \mathrm{C}$. The reaction was stirred for $1 \mathrm{~h}$, and then treated with a solution of $8(1.80 \mathrm{~g}, 4.90 \mathrm{mmol})$ in THF $(22.4 \mathrm{ml})$. The reaction was continued for $1 \mathrm{~h}$ at $-30{ }^{\circ} \mathrm{C}$, and methyl chloroformate $(2.88 \mathrm{ml}, 36.8 \mathrm{mmol})$ was added at $-20^{\circ} \mathrm{C}$. The reaction was quenched with saturated aqueous $\mathrm{NH}_{4} \mathrm{Cl}$ solution, and the aqueous phase was extracted with EtOAc. The combined organic extracts were dried over anhydrous $\mathrm{Na}_{2} \mathrm{SO}_{4}$ and concentrated in vacuo. Flash chromatography (30:1 hexanes/EtOAc) afforded the corresponding carbonate $\mathbf{1 0}(3.23 \mathrm{~g})$ including unseparable impurities.

To a solution of palladium acetylacetonate $(73.6 \mathrm{mg}, 245 \mu \mathrm{mol})$ and tributylphosphine (256 $\mu \mathrm{l}, 979 \mu \mathrm{mol})$ in toluene $(22.4 \mathrm{ml})$ were added a solution of the crude carbonate $\mathbf{1 0}$ in toluene $(22.4 \mathrm{ml})$ and ammonium formate $(771 \mathrm{mg}, 12.2 \mathrm{mmol})$. The resulting solution was warmed up to $70{ }^{\circ} \mathrm{C}$ and stirred for $1 \mathrm{hr}$. The reaction mixture was concentrated in vacuo and purified by column chromatography (50:1 hexanes/EtOAc) to afford $11(2.44 \mathrm{~g}, 90 \%$ for 2 steps $)$ as a colorless oil. $[\alpha]_{\mathrm{D}}^{24}-3.1^{\circ}\left(c 0.29, \mathrm{CHCl}_{3}\right)$; IR (KBr) 2910, 2898, 2856, 1513, 1459, 1112, 1106, $1091 \mathrm{~cm}^{-1}$; ${ }^{1} \mathrm{H}-\mathrm{NMR}(270 \mathrm{MHz}$, $\left.\mathrm{CDCl}_{3}\right) \delta 0.95(\mathrm{~d}, 6 \mathrm{H}, J=6.6 \mathrm{~Hz}), 0.98(\mathrm{~m}, 1 \mathrm{H}), 1.08(\mathrm{~s}, 9 \mathrm{H}), 1.17(\mathrm{~d}, 3 \mathrm{H}, J=6.9 \mathrm{~Hz})$, $1.46(\mathrm{~m}, 1 \mathrm{H}), 1.68(\mathrm{~m}, 1 \mathrm{H}), 1.75(\mathrm{~m}, 1 \mathrm{H}), 1.97(\mathrm{ddd}, 1 \mathrm{H}, J=16.2,7.3,1.7 \mathrm{~Hz}), 2.15$ (ddd, $1 \mathrm{H}, J=16.5,4.9,1.7 \mathrm{~Hz}), 2.70(\mathrm{~m}, 1 \mathrm{H}), 3.30(\mathrm{~m}, 1 \mathrm{H}), 3.48(\mathrm{~m}, 3 \mathrm{H}), 3.81(\mathrm{~s}, 3 \mathrm{H})$, $4.48(\mathrm{~s}, 2 \mathrm{H}), 6.89(\mathrm{~d}, 2 \mathrm{H}, J=8.6 \mathrm{~Hz}), 7.28(\mathrm{~d}, 2 \mathrm{H}, J=8.6 \mathrm{~Hz}), 7.37-7.71(\mathrm{~m}, 10 \mathrm{H}) ;{ }^{13} \mathrm{C}-$ $\operatorname{NMR}\left(67.5 \mathrm{MHz}, \mathrm{CDCl}_{3}\right) \delta 17.5,18.3,19.3,20.1,25.9,26.7,26.9,30.1,33.2,40.1$, 55.2, 69.0, 72.6, 74.3, 79.7, 83.0, 113.7, 127.5, 129.2, 129.5, 130.5, 134.0, 135.6, 159.1; HRMS (FAB, $m$-NBA) $[\mathrm{M}+\mathrm{H}]^{+}$calcd for $\mathrm{C}_{36} \mathrm{H}_{48} \mathrm{O}_{3} \mathrm{Si} 556.3372$, found 556.3364.

(2S,6R,8S)-9-(tert-Butyldiphenylsilyloxy)-2,6,8-trimethylnon-3-yn-1-ol (12)

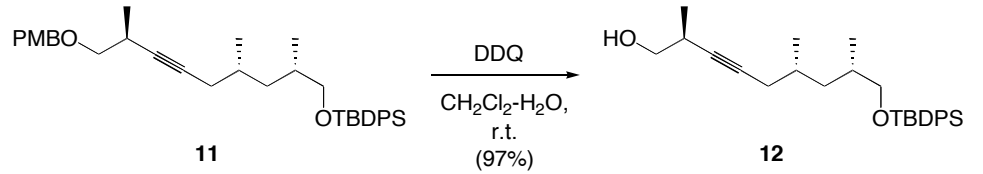

To a solution of $\mathbf{1 1}(649 \mathrm{mg}, 1.16 \mathrm{mmol})$ in $\mathrm{CH}_{2} \mathrm{Cl}_{2}(12 \mathrm{ml})$ and $\mathrm{H}_{2} \mathrm{O}(1.2 \mathrm{ml})$ was added DDQ (344 mg, $1.51 \mathrm{mmol})$ at room temperature. The reaction mixture was stirred for 30 min, and then diluted with water. The aqueous phase was extracted with EtOAc. The 
combined organic extracts were dried over anhydrous $\mathrm{Na}_{2} \mathrm{SO}_{4}$ and concentrated in vacuo. Flash chromatography (30:1 hexanes/EtOAc) afforded 12 (494 mg, 97\%) as a colorless oil. $[\alpha]_{D}^{24}-11.6^{\circ}\left(c 0.26, \mathrm{CHCl}_{3}\right)$; IR (KBr) 3644, 2964, 2960, 2931, 2325, $1112,761 \mathrm{~cm}^{-1}$; ${ }^{1} \mathrm{HNMR}\left(270 \mathrm{MHz}, \mathrm{CDCl}_{3}\right) \delta 0.96(\mathrm{~d}, 6 \mathrm{H}, J=6.6 \mathrm{~Hz}), 0.98(\mathrm{~m}, 1 \mathrm{H})$, $1.08(\mathrm{~s}, 9 \mathrm{H}), 1.14(\mathrm{~d}, 3 \mathrm{H}, J=6.9 \mathrm{~Hz}), 1.47(\mathrm{~m}, 1 \mathrm{H}), 1.70(\mathrm{~m}, 1 \mathrm{H}), 1.75(\mathrm{~m}, 1 \mathrm{H}), 1.98$ (ddd, $1 \mathrm{H}, J=16.5,6.9,1.7 \mathrm{~Hz}), 2.17(\mathrm{ddd}, 1 \mathrm{H}, J=16.1,5.0,1.7 \mathrm{~Hz}), 2.64(\mathrm{~m}, 1 \mathrm{H})$, $3.49(\mathrm{~m}, 4 \mathrm{H}), 7.37-7.71(\mathrm{~m}, 10 \mathrm{H}) ;{ }^{13} \mathrm{C}-\mathrm{NMR}\left(67.5 \mathrm{MHz}, \mathrm{CDCl}_{3}\right) \delta 17.3,17.5,19.3$, 20.2, 25.8, 26.9, 29.5, 30.0, 33.2, 40.1, 67.1, 68.9, 81.1, 82.4, 127.6, 129.5, 134.0, 135.6; HRMS (FAB, $m$-NBA) $[\mathrm{M}+\mathrm{Na}]^{+}$calcd for $\mathrm{C}_{28} \mathrm{H}_{40} \mathrm{O}_{2} \mathrm{SiNa} 459.2695$, found 459.2677 .

(2S,6R,8S,Z)-9-(tert-Butyldiphenylsilyloxy)-2,4,6,8-tetramethylnon-3-en-1-ol (13)

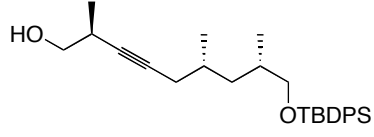

12

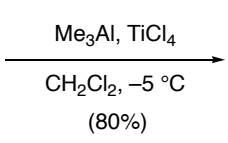

$(80 \%)$

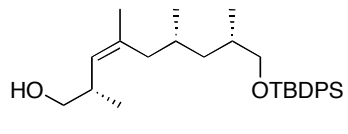

13

To a solution of trimethylaluminium (1.0 M solution in hexanes, $6.20 \mathrm{ml}, 6.20 \mathrm{mmol})$ was added a solution of $12(673 \mathrm{mg}, 1.54 \mathrm{mmol})$ in $\mathrm{CH}_{2} \mathrm{Cl}_{2}(3 \mathrm{ml})$ at $0{ }^{\circ} \mathrm{C}$. The mixture was allowed to warm to room temperature, stirred for $20 \mathrm{~min}$, and then cooled to -15 ${ }^{\circ} \mathrm{C}$. The resulting solution was treated with titanium tetrachloride $(1.0 \mathrm{M}$ solution in $\mathrm{CH}_{2} \mathrm{Cl}_{2}, 3.10 \mathrm{ml}, 3.10 \mathrm{mmol}$ ) and continued for $15 \mathrm{~min}$. The reaction was cooled to -40 ${ }^{\circ} \mathrm{C}$ and then cautiously quenched with ice-cooled $\mathrm{MeOH}(3 \mathrm{ml})$ followed by Celite (10 g) and $\mathrm{Na}_{2} \mathrm{SO}_{4} \cdot 10 \mathrm{H}_{2} \mathrm{O}(10 \mathrm{~g})$. The mixuture was allowed to warm to room temperature and stirred for $2 \mathrm{hr}$. The mixture was filtered through a pad of Celite and the filtrate was concentrated in vacuo. The residue was purified by flash chromatography (40:1 hexanes/EtOAc) to afford $13(557 \mathrm{mg}, 80 \%)$ as a colorless oil. $[\alpha]^{24}-9.1^{\circ}(c 0.17$, $\mathrm{CHCl}_{3}$ ); IR (KBr) 3444, 2960, 2931, 2896, 2869, 1473, 1459, 1450, 1427, $1105 \mathrm{~cm}^{-1}$; ${ }^{1} \mathrm{H}-\mathrm{NMR}\left(270 \mathrm{MHz}, \mathrm{CDCl}_{3}\right) \delta 0.77(\mathrm{~d}, 3 \mathrm{H}, J=6.3 \mathrm{~Hz}), 0.90(\mathrm{~d}, 3 \mathrm{H}, J=6.6 \mathrm{~Hz}), 0.95$ $(\mathrm{d}, 3 \mathrm{H}, J=6.6 \mathrm{~Hz}), 0.98(\mathrm{~m}, 1 \mathrm{H}), 1.06(\mathrm{~s}, 9 \mathrm{H}), 1.34(\mathrm{~m}, 1 \mathrm{H}), 1.66(\mathrm{~s}, 3 \mathrm{H}), 1.70(\mathrm{~m}, 1 \mathrm{H})$, $1.79(\mathrm{~m}, 1 \mathrm{H}), 1.90(\mathrm{~m}, 2 \mathrm{H}), 2.62(\mathrm{~m}, 1 \mathrm{H}), 3.28(\mathrm{dd}, 1 \mathrm{H}, J=10.2,8.2 \mathrm{~Hz}), 3.43(\mathrm{~m}, 2 \mathrm{H})$, $3.52(\mathrm{dd}, 1 \mathrm{H}, J=9.8,5.1 \mathrm{~Hz}), 4.93(\mathrm{~d}, 1 \mathrm{H}, J=9.6 \mathrm{~Hz}), 7.30-7.70(\mathrm{~m}, 10 \mathrm{H}) ;{ }^{13} \mathrm{C}-\mathrm{NMR}$ $\left(67.5 \mathrm{MHz}, \mathrm{CDCl}_{3}\right) \delta 17.1,17.7,19.3,19.7,23.7,27.0,28.3,33.2,35.2,39.5,41.5$, $67.9,68.9,127.5,128.8,129.5,134.0,135.5,137.0$; HRMS (FAB, $m-\mathrm{NBA})[\mathrm{M}+\mathrm{Na}]^{+}$ calcd for $\mathrm{C}_{29} \mathrm{H}_{44} \mathrm{O}_{2} \mathrm{SiNa} 475.3008$, found: 475.3022 .

(2S,4R,6S,8S)-9-(tert-Butyldiphenylsilyloxy)-2,4,6,8-tetramethylnonan-1-ol (14)

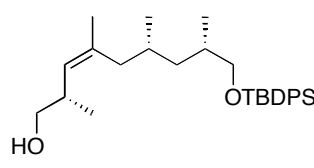

13
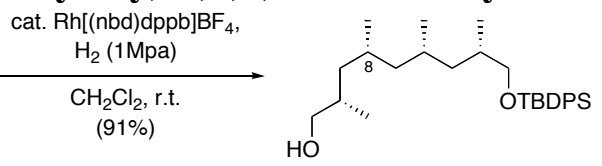

14

A solution of $13(941 \mathrm{mg}, 2.08 \mathrm{mmol})$ and $\mathrm{Rh}[(\mathrm{nbd}) \mathrm{dppb}] \mathrm{BF}_{4}(148 \mathrm{mg}, 0.208 \mathrm{mmol})$ in $\mathrm{CH}_{2} \mathrm{Cl}_{2}(4 \mathrm{ml})$ was stirred under $1 \mathrm{Mpa}$ of $\mathrm{H}_{2}$ gas for $2 \mathrm{hr}$. The resulting solution was filtered through a short plug of silica gel and concentrated in vacuo. The residue was purified by flash chromatography (40:1 hexanes/EtOAc) to afford $\mathbf{1 4}(929 \mathrm{mg}, 91 \%)$ as a colorless oil. $[\alpha]^{25}-16.6^{\circ}\left(c 0.15, \mathrm{CHCl}_{3}\right)$; IR (KBr) 3425, 2958, 2929, 2858, 1461, 1427, 1112, $1081 \mathrm{~cm}^{-1} ;{ }^{1} \mathrm{H}-\mathrm{NMR}\left(270 \mathrm{MHz}, \mathrm{CDCl}_{3}\right) \delta 0.83(\mathrm{~d}, 3 \mathrm{H}, J=6.3 \mathrm{~Hz}), 0.84$ (d, $3 \mathrm{H}, J=6.6 \mathrm{~Hz}), 0.85(\mathrm{~m}, 1 \mathrm{H}), 0.89(\mathrm{~d}, 3 \mathrm{H}, J=6.9 \mathrm{~Hz}), 0.93(\mathrm{~m}, 1 \mathrm{H}), 0.95(\mathrm{~d}, 3 \mathrm{H}, J=$ 
$6.6 \mathrm{~Hz}), 1.03(\mathrm{~m}, 2 \mathrm{H}), 1.07(\mathrm{~s}, 9 \mathrm{H}), 1.12(\mathrm{~m}, 1 \mathrm{H}), 1.33(\mathrm{~m}, 1 \mathrm{H}), 1.52(\mathrm{~m}, 1 \mathrm{H}), 1.57(\mathrm{~m}$, $1 \mathrm{H}), 1.70(\mathrm{~m} 2 \mathrm{H}), 3.36-3.59(\mathrm{~m}, 4 \mathrm{H}), 7.30-7.74(\mathrm{~m}, 10 \mathrm{H}) ;{ }^{13} \mathrm{C}-\mathrm{NMR}(67.5 \mathrm{MHz}$, $\left.\mathrm{CDCl}_{3}\right) \delta 16.0,18.0,19.2,20.2,20.5,26.8,27.0,27.4,33.1,33.2,39.9,41.5,46.1,68.8$, 69.2, 127.5, 129.4, 134.0, 135.5; HRMS (FAB, $m$-NBA) $[\mathrm{M}+\mathrm{Na}]^{+}$calcd for $\mathrm{C}_{29} \mathrm{H}_{46} \mathrm{O}_{2} \mathrm{SiNa}$ 477.3164, found 477.3266.
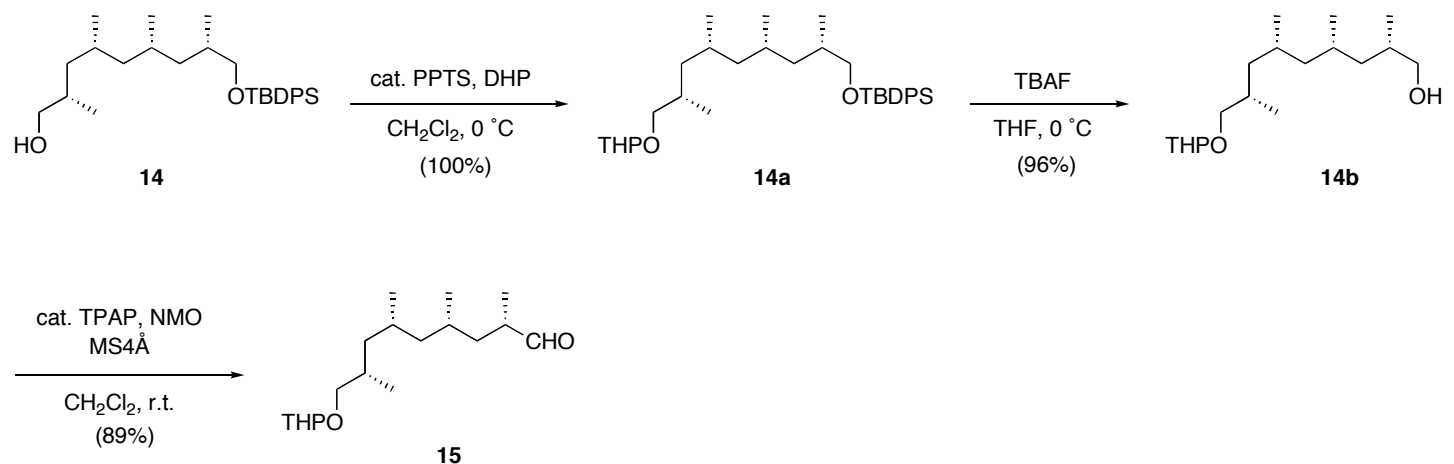

tert-Butyldiphenyl $[(2 S, 4 S, 6 R, 8 S)-2,4,6,8$-tetramethyl-9-(tetrahydro-2H-pyran-2yloxy)nonyloxy] silane (14a)

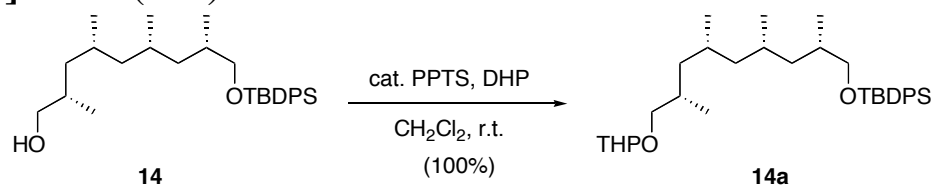

To a solution of $\mathbf{1 4}(929 \mathrm{mg}, 2.05 \mathrm{mmol})$ in $\mathrm{CH}_{2} \mathrm{Cl}_{2}(20 \mathrm{ml})$ were added dihydropyrane $(1.85 \mathrm{ml}, 20.5 \mathrm{mmol})$ and PPTS $(51.0 \mathrm{mg}, 0.205 \mathrm{mmol})$. The resulting solution was stirred at room temperature for $2 \mathrm{hr}$. The reaction was quenched with saturated aqueous $\mathrm{NaHCO}_{3}$ solution and the aqueous phase was extracted with $\mathrm{CH}_{2} \mathrm{Cl}_{2}$. The combined organic extracts were dried over anhydrous $\mathrm{Na}_{2} \mathrm{SO}_{4}$ and concentrated in vacuo. Flash chromatography (50:1 hexanes/EtOAc) afforded 14a $(1.10 \mathrm{~g}, 100 \%)$ as a colorless oil. $[\alpha]^{25}-10.5^{\circ}\left(c 0.19, \mathrm{CHCl}_{3}\right)$; IR (KBr) 3450, 2956, 2929, 2366, 1473, 1461, 1079 $\mathrm{cm}^{-1}$; ${ }^{1} \mathrm{HNMR}\left(270 \mathrm{MHz}, \mathrm{CDCl}_{3}\right) \delta 0.82(\mathrm{~d}, 3 \mathrm{H}, J=6.6 \mathrm{~Hz}), 0.83(\mathrm{~d}, 3 \mathrm{H}, J=6.6 \mathrm{~Hz})$, $0.84(\mathrm{~m}, 1 \mathrm{H}), 0.91(\mathrm{~d}, 3 \mathrm{H}, J=6.3 \mathrm{~Hz}), 0.92(\mathrm{~m}, 1 \mathrm{H}), 0.94(\mathrm{~d}, 3 \mathrm{H}, J=6.6 \mathrm{~Hz}), 1.03(\mathrm{~m}$, 2H), $1.07(\mathrm{~s}, 9 \mathrm{H}), 1.09(\mathrm{~m}, 1 \mathrm{H}), 1.29(\mathrm{~m}, 1 \mathrm{H}), 1.47-1.80(\mathrm{~m}, 10 \mathrm{H}), 3.17(\mathrm{~m}, 1 \mathrm{H}), 3.39-$ $3.61(\mathrm{~m}, 4 \mathrm{H}), 3.87(\mathrm{~m}, 1 \mathrm{H}), 4.58(\mathrm{~m}, 1 \mathrm{H}), 7.35-7.71(\mathrm{~m}, 10 \mathrm{H}) ;{ }^{13} \mathrm{C}-\mathrm{NMR}(67.5 \mathrm{MHz}$, $\left.\mathrm{CDCl}_{3}\right) \delta 16.7,16.8,17.9,18.0,19.3,19.5,19.6,20.3,20.6,20.7,25.6,26.8,27.0,27.1$, 27.4, 30.7, 30.8, 30.9, 33.1, 40.6, 41.4, 46.1, 62.0, 62.2, 68.8, 73.8, 74.0, 98.6, 99.0, 127.5, 129.5, 134.1, 135.6; HRMS (FAB, $m$-NBA) $[\mathrm{M}+\mathrm{Na}]^{+}$calcd for $\mathrm{C}_{34} \mathrm{H}_{54} \mathrm{O}_{3} \mathrm{SiNa}$ 561.3739 , found 561.3741 . 
$(2 S, 4 S, 6 R, 8 S)-2,4,6,8$-Tetramethyl-9-(tetrahydro-2H-pyran-2-yloxy)nonan-1-ol (14b)

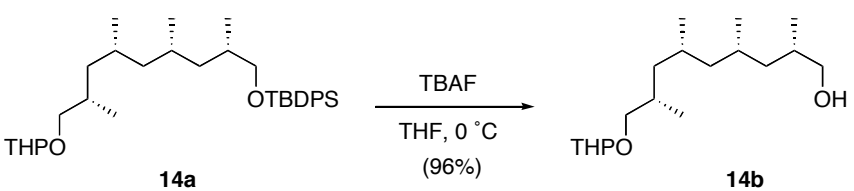

To a stirred solution of $\mathbf{1 4 a}(1.10 \mathrm{~g}, 2.05 \mathrm{mmol})$ in THF $(20 \mathrm{ml})$ was added TBAF (1.0 $\mathrm{M}$ solution in THF, $5.00 \mathrm{ml}, 5.00 \mathrm{mmol}$ ). The resulting solution was stirred for $3 \mathrm{hr}$, diluted with saturated aqueous $\mathrm{NH}_{4} \mathrm{Cl}$ solution, and extracted with EtOAc. The combined organic extracts were dried over anhydrous $\mathrm{Na}_{2} \mathrm{SO}_{4}$ and concentrated in vacuo. Flash chromatography (10:1 hexanes/EtOAc) afforded 14b (590 mg, 96\%) as a colorless oil. $[\alpha]_{\mathrm{D}}^{25}-13.7^{\circ}\left(c 0.29, \mathrm{CHCl}_{3}\right)$; IR (KBr) 3422, 2957, 2922, 2871, 2359, 1457, 1377, 1261, 1201, $1118 \mathrm{~cm}^{-1} ;{ }^{1} \mathrm{H}-\mathrm{NMR}\left(270 \mathrm{MHz}, \mathrm{CDCl}_{3}\right) \delta 0.81(\mathrm{~d}, 3 \mathrm{H}, J=6.9$ $\mathrm{Hz}), 0.84(\mathrm{~d}, 3 \mathrm{H}, J=7.3 \mathrm{~Hz}), 0.89(\mathrm{~d}, 3 \mathrm{H}, J=6.0 \mathrm{~Hz}), 0.90(\mathrm{~d}, 3 \mathrm{H}, J=6.9 \mathrm{~Hz}), 0.92$ $(\mathrm{m}, 2 \mathrm{H}), 1.01-1.32(\mathrm{~m}, 4 \mathrm{H}), 1.48-1.86(\mathrm{~m}, 10 \mathrm{H}), 3.15(\mathrm{~m}, 1 \mathrm{H}), 3.34(\mathrm{dd}, 1 \mathrm{H}, J=10.2$, $6.9 \mathrm{~Hz}), 3.41-3.58(\mathrm{~m}, 3 \mathrm{H}), 3.84(\mathrm{~m}, 1 \mathrm{H}), 4.54(\mathrm{~m}, 1 \mathrm{H}) ;{ }^{13} \mathrm{C}-\mathrm{NMR}\left(67.5 \mathrm{MHz}, \mathrm{CDCl}_{3}\right)$ $16.7,16.8$, 17.4, 17.5, 19.4, 19.6, 20.3, 20.6, 20.8, 25.5, 27.1, 27.2, 27.4, 30.6, 30.7, 30.8, 33.0, 40.5, 41.3, 45.8, 62.0, 68.1, 68.2, 73.6, 73.9, 98.6, 99.0; HRMS (FAB, $m$ NBA) $[\mathrm{M}+\mathrm{H}]^{+}$calcd for $\mathrm{C}_{18} \mathrm{H}_{37} \mathrm{O}_{3} 301.2742$, found 301.2746 .

$(2 R, 4 S, 6 R, 8 S)-2,4,6,8$-Tetramethyl-9-(tetrahydro-2H-pyran-2-yloxy)nonanal (15)

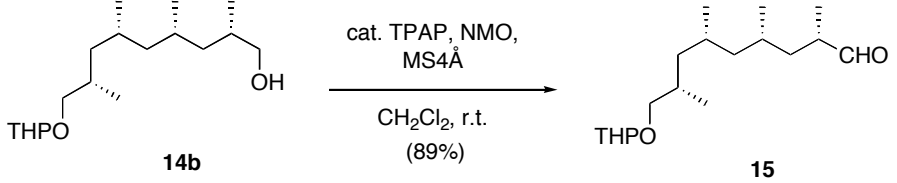

To a solution of $\mathbf{1 4 b}(389 \mathrm{mg}, 1.30 \mathrm{mmol})$ in $\mathrm{CH}_{2} \mathrm{Cl}_{2}(13 \mathrm{ml})$ were added MS4 $\mathrm{A}(1 \mathrm{~g})$, NMO (321 mg, $2.70 \mathrm{mmol})$, and TPAP $(24.0 \mathrm{mg}, 68.5 \mu \mathrm{mol})$. The reaction was stirred at room temperature for $30 \mathrm{~min}$, and then filtered through a silica pad. After evaporation of the filtrate, the residue was purified by flash chromatography (40:1 hexanes/EtOAc) to afford 15 (344 mg, 89\%) as a colorless oil. $[\alpha]_{\mathrm{D}}^{25}+1.0^{\circ}\left(c 0.14, \mathrm{CHCl}_{3}\right)$; IR $(\mathrm{KBr})$ 2955, 2873, 1734, 1488, 1457, 1261, $1031 \mathrm{~cm}^{-1} ;{ }^{1} \mathrm{HNMR}\left(270 \mathrm{MHz}, \mathrm{CDCl}_{3}\right) \delta 0.81(\mathrm{~d}$, $3 \mathrm{H}, J=6.3 \mathrm{~Hz}), 0.87(\mathrm{~d}, 6 \mathrm{H}, J=6.3 \mathrm{~Hz}), 1.00-1.07(\mathrm{~m}, 5 \mathrm{H}), 1.16(\mathrm{~d}, 3 \mathrm{H}, J=6.9 \mathrm{~Hz})$, 1.47-1.87 (m, 10H), $2.56(\mathrm{~m}, 1 \mathrm{H}), 3.16(\mathrm{~m}, 1 \mathrm{H}), 3.40-3.58(\mathrm{~m}, 2 \mathrm{H}), 3.85(\mathrm{~m}, 1 \mathrm{H}), 4.56$ $(\mathrm{m}, 1 \mathrm{H}), 9.57(\mathrm{~d}, 1 \mathrm{H}, J=2.3 \mathrm{~Hz}) ;{ }^{13} \mathrm{C}-\mathrm{NMR}\left(67.5 \mathrm{MHz}, \mathrm{CDCl}_{3}\right) \delta 16.8,16.9,18.0$, 19.4, 19.6, 19.8, 20.1, 25.5, 27.0, 27.1, 28.0, 30.6, 30.7, 30.8, 37.2, 40.9, 41.1, 45.9, 62.0, 62.2, 73.6, 73.9, 98.6, 99.0, 182.7; HRMS (FAB, $m$-NBA) $[\mathrm{M}+\mathrm{Na}]^{+}$calcd for $\mathrm{C}_{18} \mathrm{H}_{34} \mathrm{O}_{3} \mathrm{Na} 321.2405$, found 321.2391.

(4R)-4-Benzyl-3-[(3S,4S,6S,8R,10S)-3-hydroxy-4,6,8,10-tetramethyl-11-(tetrahydro$2 H$-pyran-2-yloxy)undecanoyl]oxazolidin-2-one (16)

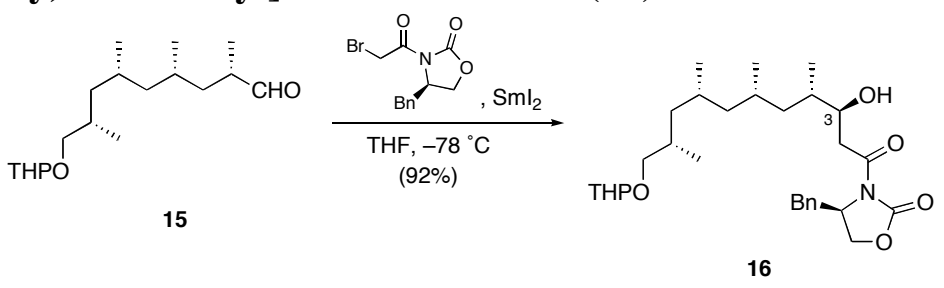


To a solution of samarium iodide $(0.1 \mathrm{M}$ solution in THF, $58.0 \mathrm{ml}, 5.80 \mathrm{mmol})$ was added a solution of 15 (344 $\mathrm{mg}, 1.15 \mathrm{mmol})$ and $(R)$-4-benzyl-3-bromoacetyl-2oxazolidinone $(376 \mathrm{mg}, 1.26 \mathrm{mmol})$ in THF $(11 \mathrm{ml})$ at $-78^{\circ} \mathrm{C}$. The reaction was stirred for $20 \mathrm{~min}$ and then treated with hexane $(100 \mathrm{ml})$ followed by silica gel $(50 \mathrm{~g})$. The mixture was allowed to warm to room temperature and stirred for $30 \mathrm{~min}$. The mixture was filtered through a short plug of silica gel and concentrated in vacuo. The residue was purified by flash chromatography (5:1 hexanes/EtOAc) to afford $\mathbf{1 6}(551 \mathrm{mg}, 92 \%)$ along with its epimer $(40.0 \mathrm{mg}, 6.2 \%) .[\alpha]_{\mathrm{D}}^{26}-43.8^{\circ}\left(c 0.25, \mathrm{CHCl}_{3}\right)$; IR $(\mathrm{KBr}) 3566$, 2955, 1785, 1698, 1456, 1386, 1199, $1030 \mathrm{~cm}^{-1} ;{ }^{1} \mathrm{H}-\mathrm{NMR}\left(270 \mathrm{MHz}, \mathrm{CDCl}_{3}\right) \delta 0.85(\mathrm{~d}$, $3 \mathrm{H}, J=5.9 \mathrm{~Hz}), 0.87(\mathrm{~d}, 3 \mathrm{H}, J=5.3 \mathrm{~Hz}), 0.89(\mathrm{~d}, 3 \mathrm{H}, J=5.0 \mathrm{~Hz}), 0.93(\mathrm{~d}, 3 \mathrm{H}, J=6.3$ $\mathrm{Hz}), 0.98-1.14(\mathrm{~m}, 3 \mathrm{H}), 1.25(\mathrm{~m}, 2 \mathrm{H}), 1.42-174(\mathrm{~m}, 10 \mathrm{H}), 1.83(\mathrm{~m}, 1 \mathrm{H}), 2.79$ (dd, $1 \mathrm{H}, J$ $=13.2,9.6 \mathrm{~Hz}), 3.07(\mathrm{~d}, 2 \mathrm{H}, J=6.3 \mathrm{~Hz}), 3.19(\mathrm{~m}, 1 \mathrm{H}), 3.31(\mathrm{dd}, 1 \mathrm{H}, J=13.2,2.6 \mathrm{~Hz})$, $3.48(\mathrm{~m}, 1 \mathrm{H}), 3.54(\mathrm{~m}, 1 \mathrm{H}), 3.86(\mathrm{~m}, 1 \mathrm{H}), 4.10(\mathrm{~m}, 1 \mathrm{H}), 4.21(\mathrm{~m}, 2 \mathrm{H}), 4.57(\mathrm{~m}, 1 \mathrm{H})$, $4.69(\mathrm{~m}, 1 \mathrm{H}), 7.18-7.36(\mathrm{~m}, 5 \mathrm{H}) ;{ }^{13} \mathrm{C}-\mathrm{NMR}\left(67.5 \mathrm{MHz}, \mathrm{CDCl}_{3}\right) \delta$ 14.5, 16.7, 16.8, 19.4, 19.6, 20.3, 20.7, 25.5, 27.1, 27.2, 30.6, 30.8, 30.9, 35.2, 37.9, 40.4, 40.5, 40.9, 45.6, 55.1, 62.2, 61.9, 66.3, 70.2, 70.3, 73.7, 73.9, 98.6, 99.0, 127.3, 128.9, 129.3, 135.1, 153.4, 173.1; HRMS (FAB, $m$-NBA) $[\mathrm{M}+\mathrm{Na}]^{+}$calcd for $\mathrm{C}_{30} \mathrm{H}_{47} \mathrm{O}_{6} \mathrm{NNa} 540.3301$, found 540.3309 . 
After conversion of $\mathbf{1 6}$ into the corresponding methyl ester, absolute configuration of C3 was determined by modified Mosher ester analysis.*

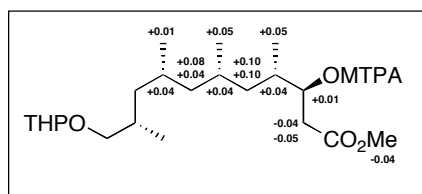

$\Delta \delta=\left(\delta_{S}-\delta_{R}\right)$ for $(R)$ - and $(S)$-MTPA derivatives

* Ohtani, I.; Kusumi, J.; Kashman, Y.; Kakisawa, H. J. Am. Chem. Soc. 1991, 113, 4092.

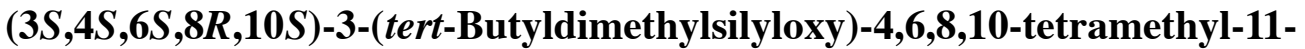
(tetrahydro-2H-pyran-2-yloxy)undecanoic acid (3)
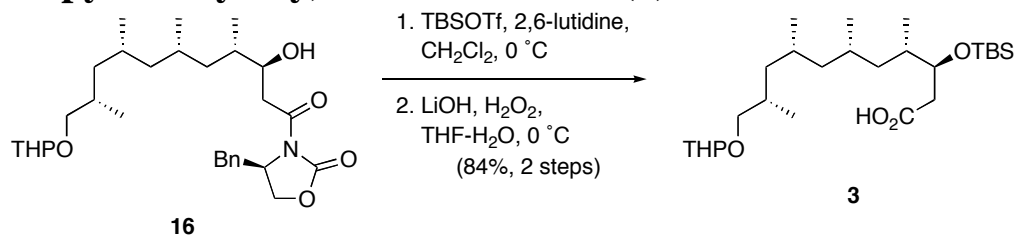

To a solution of $\mathbf{1 6}(453 \mathrm{mg}, 0.876 \mathrm{mmol})$ in $\mathrm{CH}_{2} \mathrm{Cl}_{2}(9 \mathrm{ml})$ was added 2,6-lutidine (170 $\mu 1,1.49 \mathrm{mmol})$ followed by TBSOTf $(260 \mu \mathrm{l}, 1.14 \mathrm{mmol})$ at $0{ }^{\circ} \mathrm{C}$. After $30 \mathrm{~min}$, the reaction was quenched with water and the aqueous phase was extracted with $\mathrm{CH}_{2} \mathrm{Cl}_{2}$. The combined organic extracts were dried over anhydrous $\mathrm{Na}_{2} \mathrm{SO}_{4}$ and concentrated in vacuo. This residue was employed in the next reaction without further purification.

The resulting silyl ether was dissolved in THF $(6 \mathrm{ml})$ and $\mathrm{H}_{2} \mathrm{O}(2 \mathrm{ml})$ at $0{ }^{\circ} \mathrm{C}$. Lithium hydroxide $\left(72.9 \mathrm{mg}, 1.74 \mathrm{mmol}\right.$ ) and hydrogen peroxide $\left(30 \%\right.$ solution in $\mathrm{H}_{2} \mathrm{O}, 590 \mu \mathrm{l}$, $5.21 \mathrm{mmol}$ ) were added to the solution. After $3 \mathrm{hr}$, the reaction was quenched with saturated aqueous $\mathrm{Na}_{2} \mathrm{~S}_{2} \mathrm{O}_{3}$ solution, and the aqueous phase was extracted with EtOAc. The combined organic extracts were dried over anhydrous $\mathrm{Na}_{2} \mathrm{SO}_{4}$ and concentrated in vacuo. Flash chromatography (5:1 hexanes/EtOAc) afforded 3 (347 mg, 84\% for 2 steps) as a colorless oil. $[\alpha]_{\mathrm{D}}^{27}-31.5^{\circ}\left(c 0.24, \mathrm{CHCl}_{3}\right)$; IR (KBr) 3444, 2955, 2927, 1789, 1456, 1385, 1350, 1250, $1031 \mathrm{~cm}^{-1}$; ${ }^{1} \mathrm{H}-\mathrm{NMR}\left(270 \mathrm{MHz}, \mathrm{CDCl}_{3}\right) \delta 0.02(\mathrm{~s}, 3 \mathrm{H})$, $0.05(\mathrm{~s}, 3 \mathrm{H}), 0.78-0.92(\mathrm{~m}, 23 \mathrm{H}), 0.95-0.92(\mathrm{~m}, 3 \mathrm{H}), 1.33-1.88(\mathrm{~m}, 11 \mathrm{H}), 2.42(\mathrm{~d}, 2 \mathrm{H}, J$ $=5.9 \mathrm{~Hz}), 3.15(\mathrm{~m}, 1 \mathrm{H}), 3.50(\mathrm{~m}, 2), 3.85(\mathrm{~m}, 1 \mathrm{H}), 4.06(\mathrm{~m}, 1 \mathrm{H}), 4.57(\mathrm{~m}, 1 \mathrm{H}) ;{ }^{13} \mathrm{C}-$ NMR $\left(67.5 \mathrm{MHz}, \mathrm{CDCl}_{3}\right) \delta-4.7,-4.6,15.2,16.6,16.7,18.0,19.4,19.5,20.6,20.7$, 20.8, 25.5, 25.8, 27.2, 27.3, 27.5, 30.6, 30.8, 30.9, 35.9, 39.3, 40.1, 40.4, 45.7, 61.9, $62.1,72.5,73.8,74.0,98.6,99.0,177.9$; HRMS (FAB, $m$-NBA) $[\mathrm{M}+\mathrm{H}]^{+}$calcd for $\mathrm{C}_{26} \mathrm{H}_{52} \mathrm{O}_{5} \mathrm{SiNa} 495.3481$, found 495.3489 .

\section{$(1 R, 2 R)$-Cyclopentane-1,2-diyldimethanol (17)}

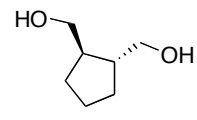

17

This diol was synthesized according to Yamamoto's procedure. (Misumi, A.; Iwanaga, K.; Furuta, K.; Yamamoto, H. J. Am. Chem. Soc. 1985, 107, 3343. )

$(1 R, 2 R)-2-[(4-M e t h o x y b e n z y l o x y)$ methyl]cyclopentanecarbaldehyde (19) 


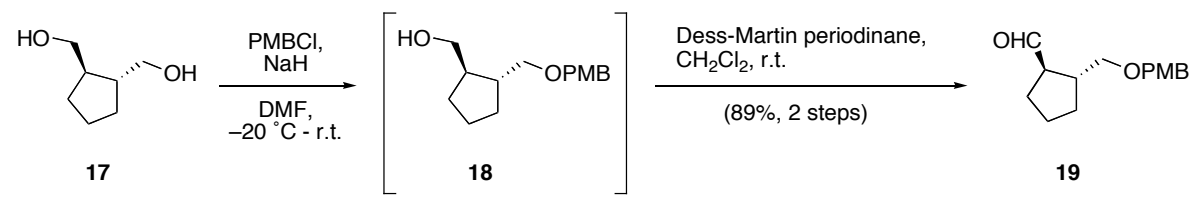

To a solution of 17 (3.63 g, $27.7 \mathrm{mmol})$ in DMF (277 ml) was added sodium hydride $\left(60 \%\right.$ in oil, $1.32 \mathrm{~g}, 31.0 \mathrm{mmol}$ ) at $-20{ }^{\circ} \mathrm{C}$. After stirring for $30 \mathrm{~min}$, the resulting suspension was treated with $\mathrm{PMBCl}(3.96 \mathrm{~mL}, 33.0 \mathrm{mmol})$ and then warmed to room temperature. The reaction was quenched with water, and the aqueous phase was extracted with EtOAc. The combined organic extracts were dried over anhydrous $\mathrm{Na}_{2} \mathrm{SO}_{4}$ and concentrated in vacuo. Flash chromatography $(7: 1$ hexanes/EtOAc) afforded the corresponding alcohol 18 including unseparable impurities.

To a solution of the alcohol in $\mathrm{CH}_{2} \mathrm{Cl}_{2}(297 \mathrm{ml})$ was added Dess-Martin periodinane $(18.7 \mathrm{~g}, 44.2 \mathrm{mmol})$. After stirring at room temperature for $30 \mathrm{~min}$, the reaction mixture was quenched with saturated aqueous $\mathrm{Na}_{2} \mathrm{~S}_{2} \mathrm{O}_{3}$ solution and saturated aqueous $\mathrm{NaHCO}_{3}$ solution, the aqueous phase was extracted with $\mathrm{CH}_{2} \mathrm{Cl}_{2}$. The combined organic extracts were dried over anhydrous $\mathrm{Na}_{2} \mathrm{SO}_{4}$ and concentrated in vacuo. Flash chromatography (20:1 hexanes/EtOAc) afforded $19\left(6.24 \mathrm{~g}, 89 \%\right.$ for 2 steps) as a colorless oil. $[\alpha]^{21}{ }_{\mathrm{D}}$ $-25.9^{\circ}$ ( c 0.27, $\mathrm{CHCl}_{3}$ ); IR (KBr) 2955, 2854, 1721, 1613, 1513, 1247, 1095, $1034 \mathrm{~cm}^{-1}$; ${ }^{1} \mathrm{H}-\mathrm{NMR}\left(270 \mathrm{MHz}, \mathrm{CDCl}_{3}\right) \delta 1.31(\mathrm{~m}, 1 \mathrm{H}), 1.57(\mathrm{~m}, 2 \mathrm{H}), 1.66-1.86(\mathrm{~m}, 3 \mathrm{H}), 2.33-2.51$ $(\mathrm{m}, 2 \mathrm{H}), 3.25(\mathrm{dd}, 1 \mathrm{H}, J=8.9,7.3 \mathrm{~Hz}), 3.39(\mathrm{dd}, 1 \mathrm{H}, J=8.9,5.6 \mathrm{~Hz}), 3.72(\mathrm{~s}, 3 \mathrm{H}), 4.36$ $(\mathrm{s}, 2 \mathrm{H}), 6.79(\mathrm{~d}, 2 \mathrm{H}, J=8.6 \mathrm{~Hz}), 7.15(\mathrm{~d}, 2 \mathrm{H}, J=8.6 \mathrm{~Hz}), 9.75(\mathrm{~d}, 1 \mathrm{H}, J=2.0 \mathrm{~Hz}) ;{ }^{13} \mathrm{C}-$ $\operatorname{NMR}\left(67.5 \mathrm{MHz}, \mathrm{CDCl}_{3}\right) \delta 24.9,26.5,29.3,41.2,55.2,55.7,72.6,72.9,113.7,129.1$, 130.4, 159.1, 203.7; HRMS (EI) $[\mathrm{M}]^{+}$calcd for $\mathrm{C}_{15} \mathrm{H}_{20} \mathrm{O}_{3}$ 248.1412, found 248.1407.

\section{(S)-1-\{(1R,2R)-2-[(4-Methoxybenzyloxy)methyl]cyclopentyl $\}$ but-3-en-1-ol (21)}

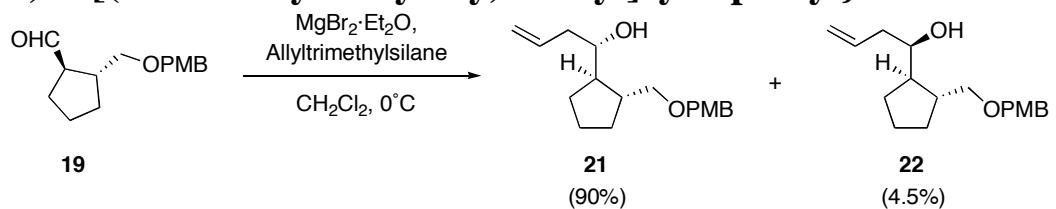

To a solution of $19(112 \mathrm{mg}, 0.45 \mathrm{mmol})$ in $\mathrm{CH}_{2} \mathrm{Cl}_{2}(4.5 \mathrm{ml})$ were added allyltrimethylsilane $(110 \mu \mathrm{l}, 0.67 \mathrm{mmol})$ and magnesium bromide diethyl etherate (116 $\mathrm{mg}, 0.45 \mathrm{mmol})$ at $0{ }^{\circ} \mathrm{C}$. The reaction was stirred for $7 \mathrm{hr}$ and then quenched with water. The aqueous phase was extracted with $\mathrm{CH}_{2} \mathrm{Cl}_{2}$. The combined organic extracts were dried over anhydrous $\mathrm{Na}_{2} \mathrm{SO}_{4}$ and concentrated in vacuo. Flash chromatography (25:1 hexanes/EtOAc) afforded 21 (117 mg, 90\%) as a colorless oil along with its epimer 22 (6.00 mg, 4.5\%). $[\alpha]^{21}{ }_{\mathrm{D}}+6.1^{\circ}\left(c 0.30, \mathrm{CHCl}_{3}\right)$; IR (KBr) 3432, 2950, 2865, 1613, 1513, 1248, 1091, $1036 \mathrm{~cm}^{-1} ;{ }^{1} \mathrm{H}-\mathrm{NMR}\left(270 \mathrm{MHz}, \mathrm{CDCl}_{3}\right) \delta 1.18-1.33(\mathrm{~m}, 2 \mathrm{H}), 1.47(\mathrm{~m}, 1 \mathrm{H})$, $1.51-1.63(\mathrm{~m}, 2 \mathrm{H}), 1.72-1.84(\mathrm{~m}, 2 \mathrm{H}), 2.02-2.17(\mathrm{~m}, 2 \mathrm{H}), 2.39(\mathrm{~m}, 1 \mathrm{H}), 3.20(\mathrm{t}, 1 \mathrm{H}, J=$ $8.9 \mathrm{~Hz}), 3.41(\mathrm{~m}, 1 \mathrm{H}), 3.53(\mathrm{dd}, 1 \mathrm{H}, J=8.9,4.6 \mathrm{~Hz}), 3.81(\mathrm{~s}, 3 \mathrm{H}), 4.47(\mathrm{~d}, 1 \mathrm{H}, J=11.9$ $\mathrm{Hz}), 4.53(\mathrm{~d}, 1 \mathrm{H}, J=11.9 \mathrm{~Hz}), 5.10(\mathrm{~m}, 2 \mathrm{H}), 5.98(\mathrm{~m}, 1 \mathrm{H}), 6.89(\mathrm{~d}, 2 \mathrm{H}, J=8.6 \mathrm{~Hz})$, $7.26(\mathrm{~d}, 2 \mathrm{H}, J=8.6 \mathrm{~Hz}) ;{ }^{13} \mathrm{C}-\mathrm{NMR}\left(67.5 \mathrm{MHz}, \mathrm{CDCl}_{3}\right) \delta 24.5,29.9,30.9,40.4,43.9$, 51.6, 55.2, 72.8, 74.2, 74.9, 113.8, 116.4, 129.4, 129.6, 135.7, 159.2; HRMS (EI) [M] ${ }^{+}$ calcd for $\mathrm{C}_{18} \mathrm{H}_{26} \mathrm{O}_{3} 290.1882$, found 290.1887 .

Stereochemical assignment of the newly created asymmetric center was achieved by Mosher's method. 


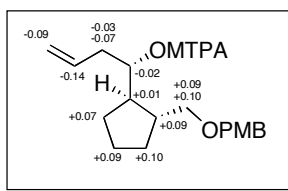

$\Delta \delta=\left(\delta_{S}-\delta_{R}\right)$ for $(R)$ - and $(S)$-MTPA ester of 21 
tert-Butyl $((S)-1-\{(1 R, 2 R)-2-[(4-m e t h o x y b e n z y l o x y) m e t h y l] c y c l o p e n t y l\} b u t-3-$ enyloxy)dimethylsilane (25)

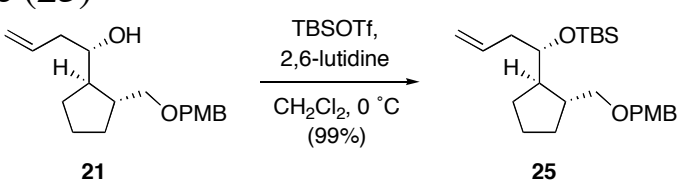

To a solution of $21(1.91 \mathrm{~g}, 6.61 \mathrm{mmol})$ in $\mathrm{CH}_{2} \mathrm{Cl}_{2}(66 \mathrm{ml})$ at $0{ }^{\circ} \mathrm{C}$ was added 2,6lutidine $(1.10 \mathrm{ml}, 9.92 \mathrm{mmol})$ followed by TBSOTf $(2.00 \mathrm{ml}, 8.59 \mathrm{mmol})$. After $1 \mathrm{hr}$, the reaction was quenched with water, and the aqueous phase was extracted with $\mathrm{CH}_{2} \mathrm{Cl}_{2}$. The combined organic extracts were dried over anhydrous $\mathrm{Na}_{2} \mathrm{SO}_{4}$ and concentrated in vacuo. Flash chromatography (50:1 hexanes/EtOAc) afforded 25 (2.65 $\mathrm{g}, 99 \%)$ as a colorless oil. $[\alpha]_{\mathrm{D}}^{22}-8.6^{\circ}\left(\mathrm{c} 0.27, \mathrm{CHCl}_{3}\right)$; IR $(\mathrm{KBr}) 2953,2856,1471$, 1249, $1095 \mathrm{~cm}^{-1} ;{ }^{1} \mathrm{H}-\mathrm{NMR}\left(270 \mathrm{MHz}, \mathrm{CDCl}_{3}\right) \delta 0.06$ (s, 6H), $0.90(\mathrm{~s}, 9 \mathrm{H}), 1.23-1.36$ $(\mathrm{m}, 2 \mathrm{H}), 1.44-1.74(\mathrm{~m}, 5 \mathrm{H}), 2.15(\mathrm{~m}, 1 \mathrm{H}), 2.26(\mathrm{~m}, 2 \mathrm{H}), 3,18(\mathrm{t}, 1 \mathrm{H}, J=8.6 \mathrm{~Hz}), 3.46$ $(\mathrm{dd}, 1 \mathrm{H}, J=8.6,4.9 \mathrm{~Hz}), 3.64(\mathrm{~m}, 1 \mathrm{H}), 3.80(\mathrm{~s}, 3 \mathrm{H}), 4.40(\mathrm{~d}, 2 \mathrm{H}, J=11.5 \mathrm{~Hz}), 4.46(\mathrm{~d}$, $2 \mathrm{H}, J=11.5 \mathrm{~Hz}), 5.01(\mathrm{~m}, 2 \mathrm{H}), 5.85(\mathrm{~m}, 1 \mathrm{H}), 6.88(\mathrm{~d}, 2 \mathrm{H}, J=8.2 \mathrm{~Hz}), 7.27(\mathrm{~d}, 2 \mathrm{H}, J=$ $8.2 \mathrm{~Hz}) ;{ }^{13} \mathrm{C}-\mathrm{NMR}\left(67.5 \mathrm{MHz}, \mathrm{CDCl}_{3}\right) \delta-4.6,-4.2,18.0,25.5,25.9,29.5,30.5,39.8$, 41.0, 46.8, 55.2, 72.6, 74.7, 75.5, 113.7, 116.5, 129.1, 130.9, 135.2, 150.0; HRMS (FAB, $m$-NBA) $[\mathrm{M}-\mathrm{H}]^{+}$calcd for $\mathrm{C}_{24} \mathrm{H}_{39} \mathrm{O}_{3} \mathrm{Si} 403.2668$, found 403.2669.

(S)-3-(tert-Butyldimethylsilyloxy)-3-\{(1R,2R)-2-[(4methoxybenzyloxy)methyl]cyclopentyl\}propanal (26)

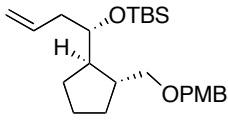

25

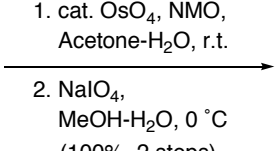

(100\%, 2 steps)

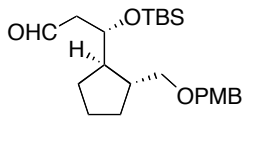

26

To a solution of $25(516 \mathrm{mg}, 1.27 \mathrm{mmol})$ in acetone $(12 \mathrm{ml})$ and $\mathrm{H}_{2} \mathrm{O}(12 \mathrm{ml})$ were added osmium tetraoxide $(32.4 \mathrm{mg}, 0.127 \mathrm{mmol})$ and NMO $(600 \mathrm{mg}, 5.10 \mathrm{mmol})$. The reaction was stirred at room temperature for $4 \mathrm{hr}$. The reaction mixture was quenched with saturated aqueous $\mathrm{Na}_{2} \mathrm{~S}_{2} \mathrm{O}_{3}$ solution and the aqueous phase was extracted with EtOAc. The combined organic extracts were dried over anhydrous $\mathrm{Na}_{2} \mathrm{SO}_{4}$ and concentrated in vacuo to give the corresponding diol as a yellow oil.

The crude diol was dissolved in $\mathrm{MeOH}(18 \mathrm{ml})$ and $\mathrm{H}_{2} \mathrm{O}(6 \mathrm{ml})$. To the reaction mixture was added sodium metaperiodate $(545 \mathrm{mg}, 2.55 \mathrm{mmol})$ at $0{ }^{\circ} \mathrm{C}$. The mixture was stirred for $2 \mathrm{hr}$, then diluted with saturated aqueous $\mathrm{Na}_{2} \mathrm{~S}_{2} \mathrm{O}_{3}$ solution. The aqueous phase was extracted with EtOAc. The combined organic extracts were dried over anhydrous $\mathrm{Na}_{2} \mathrm{SO}_{4}$ and concentrated in vacuo. Flash chromatography (40:1 hexanes/EtOAc) afforded 26 (533 mg, 100\% for 2 steps) as a colorless oil. $[\alpha]^{22}{ }_{\mathrm{D}}-14.4^{\circ}\left(c 0.36, \mathrm{CHCl}_{3}\right)$; IR (KBr) 2954, 2857, 1725, 1614, 1513, 1249, 1093, $1037 \mathrm{~cm}^{-1} ;{ }^{1} \mathrm{H}-\mathrm{NMR}(270 \mathrm{MHz}$, $\left.\mathrm{CDCl}_{3}\right) \delta 0.03(\mathrm{~s}, 3 \mathrm{H}), 0.06(\mathrm{~s}, 3 \mathrm{H}), 0.86(\mathrm{~s}, 9 \mathrm{H}), 1.32-1.75(\mathrm{~m}, 6 \mathrm{H}), 1.83(\mathrm{~m}, 1 \mathrm{H}), 2.04$ $(\mathrm{m}, 1 \mathrm{H}), 2.48(\mathrm{ddd}, 1 \mathrm{H}, J=15.8,5.9,2.6 \mathrm{~Hz}), 2.58(\mathrm{ddd}, 1 \mathrm{H}, J=15.8,4.9,1.7 \mathrm{~Hz})$, $3.31(\mathrm{~m}, 2 \mathrm{H}), 3.80(\mathrm{~s}, 3 \mathrm{H}), 4.20(\mathrm{~m}, 1 \mathrm{H}), 4.41(\mathrm{~m}, 2 \mathrm{H}), 6.87(\mathrm{~d}, 2 \mathrm{H}, J=8.6 \mathrm{~Hz}), 7.24(\mathrm{~d}$, $2 \mathrm{H}, J=8.6 \mathrm{~Hz}), 9.73(\mathrm{~m}, 1 \mathrm{H}) ;{ }^{13} \mathrm{C}-\mathrm{NMR}\left(67.5 \mathrm{MHz}, \mathrm{CDCl}_{3}\right) \delta-4.6,17.9,25.2,25.8$, 28.0, 30.3, 41.0, 48.6, 48.7, 55.2, 70.9, 72.8, 74.5, 113.7, 129.2, 130.6, 159.1, 202.5; HRMS (FAB, $m$-NBA) [M-H] ${ }^{+}$calcd for $\mathrm{C}_{23} \mathrm{H}_{37} \mathrm{O}_{4} \mathrm{Si} 405.2461$, found 405.2451. 
$(S, E)-5-($ tert-Butyldimethylsilyloxy $)-5-\{(1 R, 2 R)-2-[(4-$ methoxybenzyloxy)methyl]cyclopentyl\}pent-2-enal (27)

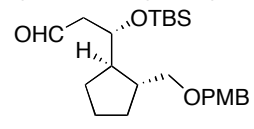

26

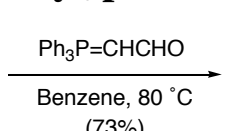

$(73 \%)$

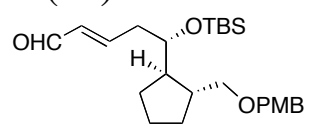

27

To a solution of $26(20.0 \mathrm{mg}, 49.2 \mu \mathrm{mol})$ in benzene $(1.0 \mathrm{ml})$ was added triphenylphosphoranylideneacetoaldehyde $(45.0 \mathrm{mg}, 98.5 \mu \mathrm{mol})$. The resulting solution was stirred at $80{ }^{\circ} \mathrm{C}$ for 2 days. The solvent was then removed in vacuo and the residue was purified by flash chromatography (30:1 hexanes/EtOAc) to afford 27 (15.5 $\mathrm{mg}$, $73 \%)$ as a yellow oil. $[\alpha]_{\mathrm{D}}^{23}+8.3^{\circ}\left(c 0.35, \mathrm{CHCl}_{3}\right)$; IR $(\mathrm{KBr}) 2953,2857,1694,1513$, 1249, 1091, $1038 \mathrm{~cm}^{-1}$; ${ }^{1} \mathrm{HNMR}\left(270 \mathrm{MHz}, \mathrm{CDCl}_{3}\right) \delta 0.04$ (s, 3H), 0.06 (s, 3H), 0.88 (s, $9 \mathrm{H}), 1.28-1.71(\mathrm{~m}, 7 \mathrm{H}), 2.08(\mathrm{~m}, 1 \mathrm{H}), 2.49(\mathrm{~m}, 2 \mathrm{H}), 3.22(\mathrm{t}, 1 \mathrm{H}, J=8.6 \mathrm{~Hz}), 3.39$ (dd, $1 \mathrm{H}, J=8.6 \mathrm{~Hz}), 3.78(\mathrm{~m}, 1 \mathrm{H}), 3.80(\mathrm{~s}, 3 \mathrm{H}), 4.39(\mathrm{~d}, 1 \mathrm{H}, J=11.9 \mathrm{~Hz}), 4.43(\mathrm{~d}, 1 \mathrm{H}, J=$ $11.9 \mathrm{~Hz}), 6.10(\mathrm{dd}, 1 \mathrm{H}, J=15.5,7.9 \mathrm{~Hz}), 6.86(\mathrm{~d}, 2 \mathrm{H}, J=8.2 \mathrm{~Hz}), 6.88(\mathrm{~m}, 1 \mathrm{H}), 7.24$ $(\mathrm{d}, 2 \mathrm{H}, J=8.2 \mathrm{~Hz}), 9.47(\mathrm{~d}, 1 \mathrm{H}, J=7.9 \mathrm{~Hz}) ;{ }^{13} \mathrm{C}-\mathrm{NMR}\left(67.5 \mathrm{MHz}, \mathrm{CDCl}_{3}\right) \delta-4.6,-4.3$, 18.0, 25.2, 25.8, 29.2, 30.3, 38.4, 41.4, 47.7, 55.2, 72.7, 74.5, 74.6, 113.7, 129.2, 130.7, 134.7, 155.2, 159.1, 193.9; HRMS (EI) $[\mathrm{M}]^{+}$calcd for $\mathrm{C}_{25} \mathrm{H}_{40} \mathrm{O}_{4} \mathrm{Si} 432.2696$, found 432.2702 . 
$(S, 2 E, 4 E)-2-B r o m o-7-(t e r t-b u t y l d i m e t h y l s i l y l o x y)-7-\{(1 R, 2 R)-2-[(4-$ methoxybenzyloxy)methyl]cyclopentyl\}hepta-2,4-dienenitrile (28)
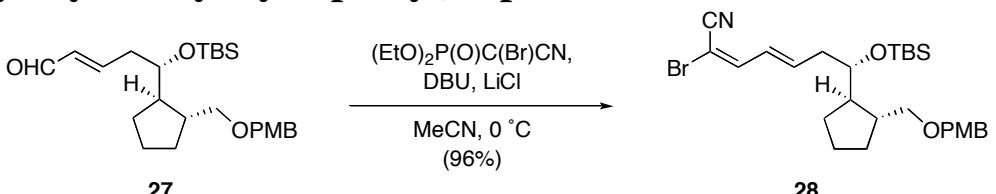

To a solution of 27 (39.8 mg, $92.1 \mathrm{mmol})$ and diethyl bromo(cyano)methylphosphate $(47.0 \mathrm{mg}, 184 \mathrm{mmol})$ in acetonitrile $(1 \mathrm{ml})$ at $0{ }^{\circ} \mathrm{C}$ were added DBU $(20.0 \mu \mathrm{l}, 138$ $\mathrm{mmol}$ ) and lithium chloride $(6.00 \mathrm{mg}, 138 \mathrm{mmol})$. The solution was stirred for $2 \mathrm{hr}$ and then diluted with saturated aqueous $\mathrm{NaHCO}_{3}$ solution. The aqueous phase was extracted with EtOAc. The combined organic extracts were dried over anhydrous $\mathrm{Na}_{2} \mathrm{SO}_{4}$ and concentrated in vacuo. Flash chromatography (60:1 hexanes/EtOAc) afforded 28 (47.3 $\mathrm{mg}, 96 \%)$ as a colorless oil. $[\alpha]^{22}{ }_{\mathrm{D}}+31.0^{\circ}\left(c 0.47, \mathrm{CHCl}_{3}\right)$; IR $(\mathrm{KBr}) 2952,2856,2217$, 1614, 1513, 1248, $1078 \mathrm{~cm}^{-1}$; ${ }^{1} \mathrm{HNMR}\left(270 \mathrm{MHz}, \mathrm{CDCl}_{3}\right) \delta 0.03(\mathrm{~s}, 3 \mathrm{H}), 0.05(\mathrm{~s}, 3 \mathrm{H})$, $0.88(\mathrm{~s}, 9 \mathrm{H}), 1.25-1.71(\mathrm{~m}, 7 \mathrm{H}), 2.07(\mathrm{~m}, 1 \mathrm{H}), 2.36(\mathrm{~m}, 2 \mathrm{H}), 3.20(\mathrm{t}, 1 \mathrm{H}, J=8.6 \mathrm{~Hz})$, $3.39(\mathrm{dd}, 1 \mathrm{H}, J=8.6,5.6 \mathrm{~Hz}), 3.69(\mathrm{~m}, 1 \mathrm{H}), 3.80(\mathrm{~s}, 3 \mathrm{H}), 4.39(\mathrm{~d}, 1 \mathrm{H}, J=11.9 \mathrm{~Hz})$, $4.44(\mathrm{~d}, 1 \mathrm{H}, J=11.9 \mathrm{~Hz}), 6.25(\mathrm{~m}, 1 \mathrm{H}), 6.36(\mathrm{dd}, 1 \mathrm{H}, J=15.2,10.6 \mathrm{~Hz}), 6.87(\mathrm{~d}, 2 \mathrm{H}, J$ $=8.6 \mathrm{~Hz}), 7.10(\mathrm{~d}, 1 \mathrm{H}, J=10.6 \mathrm{~Hz}), 7,24(\mathrm{~d}, 2 \mathrm{H}, J=8.6 \mathrm{~Hz}) ;{ }^{13} \mathrm{C}-\mathrm{NMR}(67.5 \mathrm{MHz}$, $\left.\mathrm{CDCl}_{3}\right) \delta-4.6,-4.3,17.9,25.2,25.8,29.3,30.3,38.8,41.3,47.4$, 55.1 , 72.6, 74.5, 74.7, 84.1, 113.6, 114.6, 127.6, 129.1, 130.7, 143.5, 149.7, 159.0; HRMS (FAB, $m$-NBA) $[\mathrm{M}+\mathrm{Na}]^{+}$calcd for $\mathrm{C}_{27} \mathrm{H}_{40} \mathrm{O}_{3} \mathrm{NBrSiNa} 556.1858$, found 556.1855 .

$(S, 2 E, 4 E)-2-B r o m o-7-h y d r o x y-7-\{(1 R, 2 R)-2-[(4-$ methoxybenzyloxy)methyl]cyclopentyl\}hepta-2,4-dienenitrile (4)

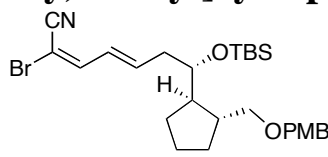

28

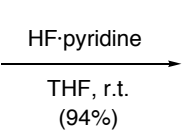

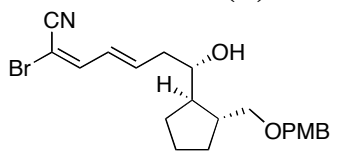

4

28 (184 mg, $345 \mu \mathrm{mol})$ was placed in a solution of HF.pyridine $(500 \mu \mathrm{l})$ in THF (500 $\mu \mathrm{l})$ and pyridine $(500 \mu \mathrm{l})$ and stirred at room temperature for 2 days. The resulting solution was filtered through a short plug of silica gel and concentrated in vacuo. The residue was purified by flash chromatography (7:1 hexanes/EtOAc) to afford 4 (135 $\mathrm{mg}$, $94 \%)$ as a colorless oil. $[\alpha]_{\mathrm{D}}^{24}+11.3^{\circ}\left(c 0.25, \mathrm{CHCl}_{3}\right)$; IR (KBr) 3406, 2949, 2222, 2216, 1613, 1513, 1248, 1075, $1035 \mathrm{~cm}^{-1} ;{ }^{1} \mathrm{H}-\mathrm{NMR}\left(270 \mathrm{MHz}, \mathrm{CDCl}_{3}\right) \delta 1.25(\mathrm{~m}, 1 \mathrm{H})$, 1.41-1.67 (m, 5H), $1.77(\mathrm{~m}, 1 \mathrm{H}), 2.04(\mathrm{~m}, 1 \mathrm{H}), 2.24(\mathrm{~m}, 1 \mathrm{H}), 2.47(\mathrm{~m}, 1 \mathrm{H}), 3.14(\mathrm{dd}$, $1 \mathrm{H}, J=10.6,8.6 \mathrm{~Hz}), 3.41(\mathrm{~m}, 1 \mathrm{H}), 3.56(\mathrm{dd}, 1 \mathrm{H}, J=8.6,4.0 \mathrm{~Hz}), 3.81(\mathrm{~s}, 3 \mathrm{H}), 4.49$ $(\mathrm{m}, 2 \mathrm{H}), 6.33-6.48(\mathrm{~m}, 2 \mathrm{H}), 6.88(\mathrm{~d}, 2 \mathrm{H}, J=8.6 \mathrm{~Hz}), 7.15(\mathrm{~m}, 1 \mathrm{H}), 7.24(\mathrm{~d}, 2 \mathrm{H}, J=8.6$ $\mathrm{Hz}) ;{ }^{13} \mathrm{C}-\mathrm{NMR}\left(67.5 \mathrm{MHz}, \mathrm{CDCl}_{3}\right) \delta 24.3,29.6,30.9,39.6,44.0,52.2,55.1,72.8,74.0$, 74.5, 83.8, 113.7, 114.6, 127.1, 129.1, 129.4, 144.3, 150.0, 159.2; HRMS (FAB, $m$ NBA) $[\mathrm{M}+\mathrm{Na}]^{+}$calcd for $\mathrm{C}_{21} \mathrm{H}_{26} \mathrm{O}_{3} \mathrm{NBrNa} 442.0994$, found 442.1002 .

$(S, 2 Z, 4 E$ ) - A 11 y l 2 - b romo-7-(tert-butyldimethylsilyloxy)-7-\{(1R,2R)-2-[(4methoxybenzyloxy)methyl]cyclopentyl\}hepta-2,4-dienoate (44) 


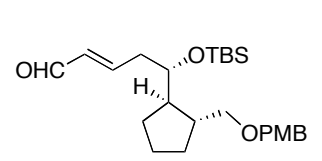

27

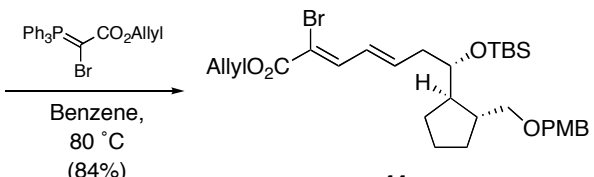

44

To a solution of 27 (461 $\mathrm{mg}, 1.07 \mathrm{mmol})$ in benzene $(10.7 \mathrm{ml})$ was added (allyloxycarbonylbromomethylidene)triphenylphosphorane $(1.04 \mathrm{~g}, 3.20 \mathrm{mmol})$ at room temperature. The reaction mixture was stirred at $70{ }^{\circ} \mathrm{C}$ for $39 \mathrm{~h}$ and concentrated in vacuo. The residue was purified by column chromatography $(70: 1$ hexanes/EtOAc) to give $44(531 \mathrm{mg}, 84 \%)$ as a yellow oil. $[\alpha]^{25}+9.4^{\circ}\left(c 0.18, \mathrm{CHCl}_{3}\right)$; IR $(\mathrm{KBr}) 2943$, 2860, 2367, 1720, 1622, 1534, 1460, 1363, 1250, 1076, 835, $773 \mathrm{~cm}^{-1} ;{ }^{1} \mathrm{H}-\mathrm{NMR}(300$ $\left.\mathrm{MHz}, \mathrm{CDCl}_{3}\right) \delta 0.03(\mathrm{~s}, 3 \mathrm{H}), 0.04(\mathrm{~s}, 3 \mathrm{H}), 0.87(\mathrm{~s}, 9 \mathrm{H}), 1.27(\mathrm{~m}, 1 \mathrm{H}), 1.40-1.75(\mathrm{~m}$, $6 \mathrm{H}), 2.08(\mathrm{~m}, 1 \mathrm{H}), 2.39(\mathrm{~m}, 2 \mathrm{H}), 3.17(\mathrm{t}, 1 \mathrm{H}, J=8.6 \mathrm{~Hz}), 3.40(\mathrm{dd}, 1 \mathrm{H}, J=8.6,5.3 \mathrm{~Hz})$, 3.77, (m, 1H), $3.79(\mathrm{~s}, 3 \mathrm{H}), 4.38(\mathrm{~d}, 1 \mathrm{H}, J=11.6 \mathrm{~Hz}), 4.44(\mathrm{~d}, 1 \mathrm{H}, J=11.6 \mathrm{~Hz}), 4.67$ (ddt, $1 \mathrm{H}, J=13.1,5.8,1.3 \mathrm{~Hz}), 4.75(\mathrm{ddt}, 1 \mathrm{H}, J=13.1,5.8,1.3 \mathrm{~Hz}), 5.28(\mathrm{dd}, 1 \mathrm{H}, J=$ $10.3,1.5 \mathrm{~Hz}), 5.39(\mathrm{dd}, 1 \mathrm{H}, J=17.3,1.5 \mathrm{~Hz}), 5.98(\mathrm{ddt}, 1 \mathrm{H}, J=17.3,10.3,5.8 \mathrm{~Hz})$, $6.35(\mathrm{~m}, 1 \mathrm{H}), 6.48(\mathrm{dd}, 1 \mathrm{H}, J=15.3,10.1 \mathrm{~Hz}), 6.86(\mathrm{~d}, 2 \mathrm{H}, J=8.7 \mathrm{~Hz}), 7.24(\mathrm{~d}, 2 \mathrm{H}, J=$ $8.7 \mathrm{~Hz}), 7.64(\mathrm{~d}, 1 \mathrm{H}, J=10.1 \mathrm{~Hz}) ;{ }^{13} \mathrm{C}-\mathrm{NMR}\left(100 \mathrm{MHz}, \mathrm{CDCl}_{3}\right) \delta-4.5,-4.2,18.1$, 25.3, 25.9, 29.4, 30.4, 39.4, 41.4, 47.4, 55.3, 66.8, 72.7, 74.6, 75.1, 111.8, 113.7, 118.7, 129.2, 129.6, 130.8, 131.7, 141.7, 144.5, 159.0, 162.9; HRMS (FAB, $m$-NBA+NaI) $[\mathrm{M}+\mathrm{Na}]^{+}$calcd for $\mathrm{C}_{30} \mathrm{H}_{45} \mathrm{BrO}_{5} \mathrm{SiNa} 615.2117$, found 615.2107. 
$(S, 2 Z, 4 E)$ - A 1 I y I

2 - b romo-7-hydroxy-7-\{(1R,2R)-2-[(4methoxybenzyloxy)methyl]cyclopentyl\}hepta-2,4-dienoate (45)

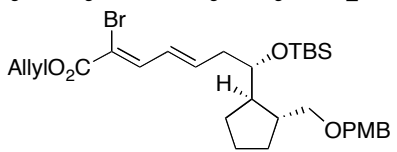

44

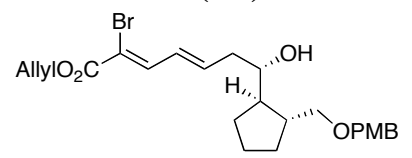

45

To a solution of $44(287 \mathrm{mg}, 0.484 \mathrm{mmol})$ in THF $(0.9 \mathrm{ml})$ at room temperature was added a HF-pyridine-THF solution (HF-pyridine : pyridine : THF = $1: 1: 1,3.6 \mathrm{ml}$ ). After stirring for $9.5 \mathrm{~h}$ at room temperature, the reaction mixture was filtered through a silica gel pad (eluate $60: 1 \mathrm{CH}_{2} \mathrm{Cl}_{2} / \mathrm{MeOH}$ ) and concentrated in vacuo. The residue was purified by column chromatography ( $8: 1$ hexanes/EtOAc) to give $45(221 \mathrm{mg}, 96 \%)$ as a yellow oil. $[\alpha]_{\mathrm{D}}^{25}=+3.2^{\circ}\left(c=0.17, \mathrm{CHCl}_{3}\right)$; IR $(\mathrm{KBr}) 3433,2924,2858,1724,1624$, 1512, 1456, 1368, 1248, 1167, $1034 \mathrm{~cm}^{-1} ;{ }^{1} \mathrm{H}-\mathrm{NMR}\left(300 \mathrm{MHz}, \mathrm{CDCl}_{3}\right) \delta 1.17(\mathrm{~m}, 2 \mathrm{H})$, 1.40-1.84 (m, 5H), $2.03(\mathrm{~m}, 1 \mathrm{H}), 2.28(\mathrm{~m}, 1 \mathrm{H}), 2.52(\mathrm{~m}, 1 \mathrm{H}), 3.14(\mathrm{dd}, 1 \mathrm{H}, J=10.6,8.7$ $\mathrm{Hz}), 3.44(\mathrm{~m}, 1 \mathrm{H}), 3.55(\mathrm{dd}, 1 \mathrm{H}, J=8.7,3.9 \mathrm{~Hz}), 3.80(\mathrm{~s}, 3 \mathrm{H}), 4.46(\mathrm{~d}, 1 \mathrm{H}, J=11.7$ $\mathrm{Hz}), 4.51(\mathrm{~d}, 1 \mathrm{H}, J=11.7 \mathrm{~Hz}), 4.68(\mathrm{ddt}, 1 \mathrm{H}, J=13.1,5.8,1.3 \mathrm{~Hz}), 4.75(\mathrm{ddt}, 1 \mathrm{H}, J=$ $13.1,5.8,1.3 \mathrm{~Hz}), 5.27(\mathrm{dd}, 1 \mathrm{H}, J=10.4,1.4 \mathrm{~Hz}), 5.38(\mathrm{dd}, 1 \mathrm{H}, J=17.3,1.4 \mathrm{~Hz}), 5.96$ (ddt, $1 \mathrm{H}, J=17.3,10.4,5.8 \mathrm{~Hz}), 6.51-6.55(\mathrm{~m}, 2 \mathrm{H}), 6.88(\mathrm{~d}, 2 \mathrm{H}, J=8.7 \mathrm{~Hz}), 7.24(\mathrm{~d}$, $2 \mathrm{H}, J=8.7 \mathrm{~Hz}), 7.70(\mathrm{~d}, 1 \mathrm{H}, J=9.7 \mathrm{~Hz}) ;{ }^{13} \mathrm{C}-\mathrm{NMR}\left(100 \mathrm{MHz}, \mathrm{CDCl}_{3}\right) \delta 24.4,29.8$, 31.1, 40.1, 44.21, 52.3, 55.3, 66.7, 73.0, 74.2, 74.9, 111.7, 113.9, 118.6, 129.1, 129.3, $129.5,131.7,142.0,145.2,159.4,162.9$; HRMS (FAB, $m-\mathrm{NBA}+\mathrm{NaI})[\mathrm{M}+\mathrm{Na}]^{+}$calcd for $\mathrm{C}_{24} \mathrm{H}_{31} \mathrm{BrO}_{5} \mathrm{Na} 501.1253$, found 501.1250. 
$(3 S, 4 S, 6 S, 8 R, 10 S)-((S, 3 E, 5 Z)-7-(A l l y l o x y)-6-b r o m o-1-\{(1 R, 2 R)-2-[(4-$ methoxybenzyloxy)methyl]cyclopentyl\}-7-oxohepta-3,5-dienyl) 3-(tertbutyldimethylsilyloxy)-4,6,8,10-tetramethyl-11-(tetrahydro-2H-pyran-2yloxy)undecanoate (46)
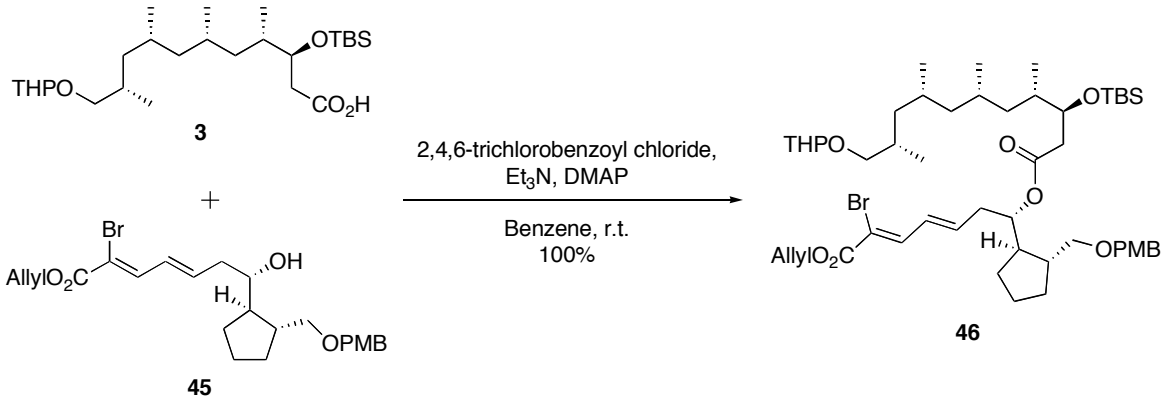

A solution of $3(229 \mathrm{mg}, 0.485 \mathrm{mmol})$ in benzene $(4.7 \mathrm{ml})$ at room temperature was treated with $\mathrm{Et}_{3} \mathrm{~N}(0.140 \mathrm{ml}, 0.970 \mathrm{mmol})$ and 2,4,6-trichlorobenzoyl chloride $(80.0 \mu \mathrm{L}$, $0.533 \mathrm{mmol}$ ) under an argon atmosphere. After $1 \mathrm{~h}$, a solution of 45 (301 $\mathrm{mg}, 0.630$ $\mathrm{mmol})$ in benzene $(5.0 \mathrm{ml})$ was added dropwise and DMAP $(77.0 \mathrm{mg}, 0.630 \mathrm{mmol})$ was then added. After stirring for $2 \mathrm{~h}$ at room temperature, the reaction was quenched with aqueous $\mathrm{NaHCO}_{3}$ solution and the resulting mixture was extracted with EtOAc. The combined organic phases were dried over anhydrous $\mathrm{Na}_{2} \mathrm{SO}_{4}$ and concentrated in vacuo. The crude product was purified by column chromatography ( $25: 1$ hexanes/EtOAc) to give $46(452 \mathrm{mg}, 100 \%)$ as a colorless oil. $[\alpha]_{\mathrm{D}}^{24}-4.8^{\circ}\left(c 0.17, \mathrm{CHCl}_{3}\right)$; IR $(\mathrm{KBr}) 2949$, 2862, 1728, 1626, 1514, 1460, 1371, 1248, 1171, 1080, 1032, 833, $775 \mathrm{~cm}^{-1}$; ${ }^{1} \mathrm{H}-\mathrm{NMR}$ $\left(300 \mathrm{MHz}, \mathrm{CDCl}_{3}\right) \delta 0.02(\mathrm{~s}, 3 \mathrm{H}), 0.05(\mathrm{~s}, 3 \mathrm{H}), 0.79-0.91(\mathrm{~m}, 23 \mathrm{H}), 0.95-1.14(\mathrm{~m}, 5 \mathrm{H})$, 1.45-1.92 (m, 16H), $2.04(\mathrm{~m}, 1 \mathrm{H}), 2.31-2.60(\mathrm{~m}, 4 \mathrm{H}), 3.17(\mathrm{~m}, 1 \mathrm{H}), 3.24(\mathrm{t}, 1 \mathrm{H}, J=8.6$ $\mathrm{Hz}), 3.37$ (dd, 1H, $J=8.6,5.6 \mathrm{~Hz}), 3.44-3.57$ (m, 2H), $3.79(\mathrm{~s}, 3 \mathrm{H}), 3.86(\mathrm{~m}, 1 \mathrm{H}), 4.06$ $(\mathrm{m}, 1 \mathrm{H}), 4.39(\mathrm{~d}, 1 \mathrm{H}, J=11.7 \mathrm{~Hz}), 4.45(\mathrm{~d}, 1 \mathrm{H}, J=11.7 \mathrm{~Hz}), 4.56(\mathrm{~m}, 1 \mathrm{H}), 4.68$ (ddt, $1 \mathrm{H}, J=13.1,5.8,1.3 \mathrm{~Hz}), 4.75(\mathrm{ddt}, 1 \mathrm{H}, J=13.1,5.8,1.3 \mathrm{~Hz}), 4.95(\mathrm{~m}, 1 \mathrm{H}), 5.28(\mathrm{dd}$, $1 \mathrm{H}, J=10.4,1.3 \mathrm{~Hz}), 5.75(\mathrm{dd}, 1 \mathrm{H}, J=17.2,1.3 \mathrm{~Hz}), 5.95(\mathrm{ddt}, 1 \mathrm{H}, J=17.2,10.4,5.8$ $\mathrm{Hz}), 6.24(\mathrm{~m}, 1 \mathrm{H}), 6.49(\mathrm{dd}, 1 \mathrm{H}, J=15.5,10.4 \mathrm{~Hz}), 6.86(\mathrm{~d}, 2 \mathrm{H}, J=8.6 \mathrm{~Hz}), 7.24(\mathrm{~d}$, $2 \mathrm{H}, J=8.6 \mathrm{~Hz}), 7.62(\mathrm{~d}, 1 \mathrm{H}, J=10.4 \mathrm{~Hz}) ;{ }^{13} \mathrm{C}-\mathrm{NMR}\left(100 \mathrm{MHz}, \mathrm{CDCl}_{3}\right) \delta-4.7,-4.3$, $14.5,16.0,16.5,16.6,18.1,19.5,19.6,20.5,20.6,20.7,20.7,25.1,25.5,25.9,27.0$, 27.1, 27.1, 27.3, 27.3, 29.7, 29.8, 30.3, 30.7, 30.9, 30.9, 33.3, 35.5, 36.8, 39.6, 39.8, 40.0, 40.9, 41.0, 41.2, 41.6, 41.6, 45.0, 45.8, 45.8, 55.2, 62.0, 62.2, 66.9, 69.3, 71.6, 71.7, 72.7, 73.9, 74.1, 75.9, 98.7, 99.0, 112.8, 113.7, 118.8, 129.0, 130.1, 130.7, 131.6, 141.2, 142.3, 159.1, 162.6, 172.7; HRMS (FAB, $m-\mathrm{NBA}+\mathrm{NaI})[\mathrm{M}+\mathrm{Na}]^{+}$calcd for $\mathrm{C}_{50} \mathrm{H}_{81} \mathrm{BrO}_{9} \mathrm{SiNa}$ 955.4731, found 955.4759.

$(3 S, 4 S, 6 S, 8 R, 10 S)-((S, 3 E, 5 Z)-7-($ Allyloxy)-6-bromo-1-\{(1R,2R)-2-[(4methoxybenzyloxy)methyl]cyclopentyl $\}$-7-oxohepta-3,5-dienyl) 3-(tertbutyldimethylsilyloxy)-11-hydroxy-4,6,8,10-tetramethylundecanoate (47)
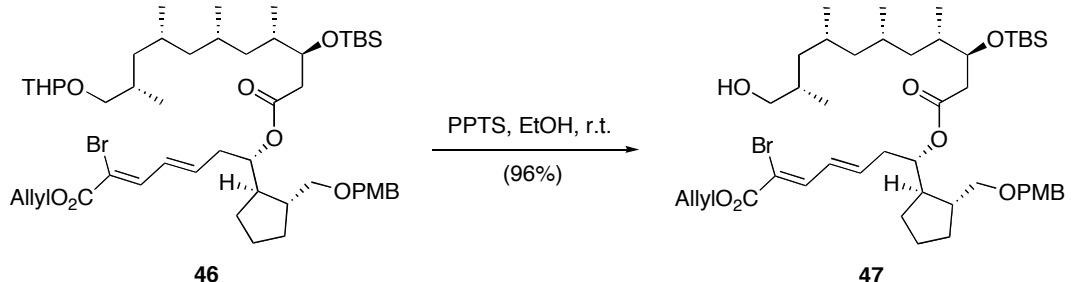

To a solution of 46 (156 mg, $0.167 \mathrm{mmol})$ in EtOH $(3.3 \mathrm{ml})$ at room temperature was 
added PPTS $(21.0 \mathrm{mg}, 84.0 \mu \mathrm{mol})$. After stirring for $16 \mathrm{~h}$ at $50{ }^{\circ} \mathrm{C}$, the reaction mixture was diluted with water and extracted with EtOAc. The combined organic phases were dried over anhydrous $\mathrm{Na}_{2} \mathrm{SO}_{4}$ and concentrated in vacuo. The crude product was purified by column chromatography $(7: 1$ hexanes/EtOAc) to give $47(136 \mathrm{mg}, 96 \%)$ as a colorless oil. $[\alpha]_{\mathrm{D}}^{25}-11.9^{\circ}\left(c 0.17, \mathrm{CHCl}_{3}\right.$ ); IR (KBr) 2952, 2862, 1726, 1626, 1514, 1460, 1371, 1248, 1171, 1090, 1134, 833, $777 \mathrm{~cm}^{-1} ;{ }^{1} \mathrm{H}-\mathrm{NMR}\left(300 \mathrm{MHz}, \mathrm{CDCl}_{3}\right) \delta 0.02$ $(\mathrm{s}, 3 \mathrm{H}), 0.05(\mathrm{~s}, 3 \mathrm{H}), 0.77-1.42(\mathrm{~m}, 28 \mathrm{H}), 1.47-1.78(\mathrm{~m}, 9 \mathrm{H}), 1.86(\mathrm{~m}, 1 \mathrm{H}), 2.05(\mathrm{~m}$, $1 \mathrm{H}), 2.30-2.60(\mathrm{~m}, 4 \mathrm{H}), 3.23(\mathrm{dd}, 1 \mathrm{H}, J=8.8,7.8 \mathrm{~Hz}), 3.34-3.46(\mathrm{~m}, 3 \mathrm{H}), 3.78(\mathrm{~s}, 3 \mathrm{H})$, $4.05(\mathrm{~m}, 1 \mathrm{H}), 4.39(\mathrm{~d}, 1 \mathrm{H}, J=11.7 \mathrm{~Hz}), 4.45(\mathrm{~d}, 1 \mathrm{H}, J=11.7 \mathrm{~Hz}), 4.66(\mathrm{ddt}, 1 \mathrm{H}, J=$ $13.1,5.8,1.3 \mathrm{~Hz}), 4.75(\mathrm{ddt}, 1 \mathrm{H}, J=13.1,5.8,1.3 \mathrm{~Hz}), 4.95(\mathrm{~m}, 1 \mathrm{H}), 5.27(\mathrm{dd}, 1 \mathrm{H}, J=$ $10.4,1.4 \mathrm{~Hz}), 5.38(\mathrm{dd}, 1 \mathrm{H}, J=17.8,1.4 \mathrm{~Hz}), 5.96(\mathrm{ddt}, 1 \mathrm{H}, J=17.8,10.4,5.8 \mathrm{~Hz}$ ), $6.23(\mathrm{dt}, 1 \mathrm{H}, J=15.1,7.4 \mathrm{~Hz}), 6.48(\mathrm{dd}, 1 \mathrm{H}, J=15.1,10.5 \mathrm{~Hz}), 6.86(\mathrm{~d}, 2 \mathrm{H}, J=8.8$ $\mathrm{Hz}), 7.24(\mathrm{~d}, 2 \mathrm{H}, J=8.8 \mathrm{~Hz}), 7.62(\mathrm{~d}, 1 \mathrm{H}, J=10.5 \mathrm{~Hz}) ;{ }^{13} \mathrm{C}-\mathrm{NMR}\left(100 \mathrm{MHz}, \mathrm{CDCl}_{3}\right) \delta$ $-4.7,-4.3,14.5,16.0,18.1,20.6,20.7,25.1,25.9,27.1,27.3,29.8,30.3,33.3,35.5$, 36.8, 39.6, 39.8, 41.2, 41.6, 45.0, 45.8, 55.3, 66.9, 69.3, 71.7, 72.7, 74.1, 76.0, 112.8, $113.7,118.8,129.1,130.1,130.6,131.6,141.2,142.3,159.1,162.7,171.7$; HRMS $(\mathrm{FAB}, m-\mathrm{NBA}+\mathrm{NaI})[\mathrm{M}+\mathrm{Na}]^{+}$calcd for $\mathrm{C}_{45} \mathrm{H}_{73} \mathrm{BrO}_{8} \mathrm{SiNa} 871.4156$, found 871.4142. 
$(3 S, 4 S, 6 S, 8 R, 10 S)-((S, 3 E, 5 Z)-7-(A l l y l o x y)-6-b r o m o-1-\{(1 R, 2 R)-2-[(4-$ methoxybenzyloxy)methyl]cyclopentyl\}-7-oxohepta-3,5-dienyl) 3-(tertbutyldimethylsilyloxy)-4,6,8,10-tetramethyl-11-oxoundecanoate (43)
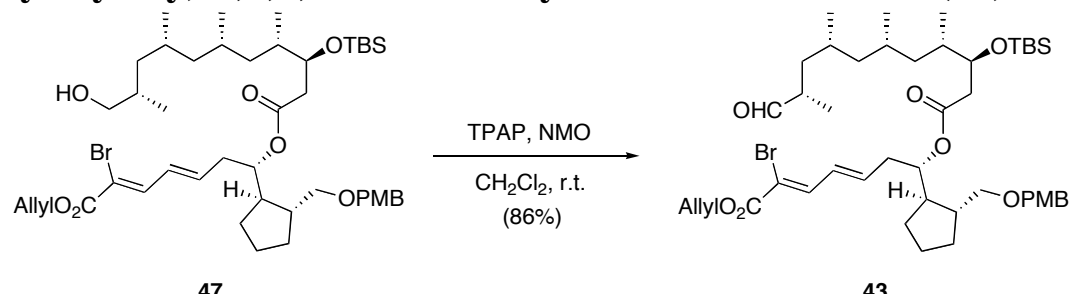

43

To a mixture of dried MS4 $\mathrm{A}$ and $47(110 \mathrm{mg}, 0.130 \mathrm{mmol})$ in $\mathrm{CH}_{2} \mathrm{Cl}_{2}$ (2.6 ml) were added NMO (30.5 mg, $0.259 \mathrm{mmol})$ and TPAP $(2.30 \mathrm{mg}, 7.00 \mu \mathrm{mol})$ at room temperature under a nitrogene atmosphere. After stirring for $0.5 \mathrm{~h}$ at room temperature, the reaction was quenched with aqueous $\mathrm{Na}_{2} \mathrm{~S}_{2} \mathrm{O}_{3}$ solution and the resulting suspension was then filtered through a pad of Celite. The filtrate was extracted with EtOAc and the combined organic layers were dried over anhydrous $\mathrm{Na}_{2} \mathrm{SO}_{4}$. The organic solvent was removed in vacuo to give the crude product. Flash chromatography (20: 1 hexanes/EtOAc) afforded aldehyde $\mathbf{4 3}(94.2 \mathrm{mg}, 86 \%)$ as a colorless oil. ${ }^{1} \mathrm{H}-\mathrm{NMR}$ (300 $\left.\mathrm{MHz}, \mathrm{CDCl}_{3}\right) \delta 0.02(\mathrm{~s}, 3 \mathrm{H}), 0.05(\mathrm{~s}, 3 \mathrm{H}), 0.80-0.99(\mathrm{~m}, 20 \mathrm{H}), 1.06(\mathrm{~d}, 3 \mathrm{H}, J=6.9 \mathrm{~Hz})$, 1.18-1.91 (m, 14H), $2.06(\mathrm{~m}, 1 \mathrm{H}), 2.31-2.59(\mathrm{~m}, 5 \mathrm{H}), 3.24(\mathrm{dd}, 1 \mathrm{H}, J=8.7,7.9 \mathrm{~Hz})$, $3.37(\mathrm{dd}, 1 \mathrm{H}, J=8.7,5.7 \mathrm{~Hz}), 3.80(\mathrm{~s}, 3 \mathrm{H}), 4.06(\mathrm{dt}, 1 \mathrm{H}, J=6.3,2.4 \mathrm{~Hz}), 4.39(\mathrm{~d}, 1 \mathrm{H}, J$ $=11.6 \mathrm{~Hz}), 4.45(\mathrm{~d}, 1 \mathrm{H}, J=11.6 \mathrm{~Hz}), 4.68(\mathrm{ddt}, 1 \mathrm{H}, J=13.1,5.8,1.3 \mathrm{~Hz}), 4.76(\mathrm{ddt}$, $1 \mathrm{H}, J=13.1,5.8,1.3 \mathrm{~Hz}), 4.96(\mathrm{~m}, 1 \mathrm{H}), 5.28(\mathrm{dd}, 1 \mathrm{H}, J=10.4 \mathrm{~Hz}, 1.3 \mathrm{~Hz}), 5.40(\mathrm{dd}$, $1 \mathrm{H}, J=17.2,1.3 \mathrm{~Hz}), 5.96(\mathrm{ddt}, 1 \mathrm{H}, J=17.2,10.4,5.8 \mathrm{~Hz}), 6.24(\mathrm{~m}, 1 \mathrm{H}), 6.49(\mathrm{dd}, 1 \mathrm{H}$, $J=15.4,10.5 \mathrm{~Hz}), 6.86(\mathrm{~d}, 2 \mathrm{H}, J=8.6 \mathrm{~Hz}), 7.24(\mathrm{~d}, 2 \mathrm{H}, J=8.6 \mathrm{~Hz}), 7.61(\mathrm{~d}, 1 \mathrm{H}, J$ $=10.5 \mathrm{~Hz}), 9.60(\mathrm{~d}, 1 \mathrm{H}, J=1.7 \mathrm{~Hz})$; HRMS $(\mathrm{FAB}, m-\mathrm{NBA}+\mathrm{NaI})[\mathrm{M}+\mathrm{Na}]^{+}$calcd for $\mathrm{C}_{45} \mathrm{H}_{71} \mathrm{BrO}_{8} \mathrm{SiNa}$ 869.3999, found 869.4013. 
(2S,4E,6Z,9S,11R,13S,15S,16S)-Allyl 16-(tert-butyldimethylsilyloxy)-8-hydroxy-2$\{(1 R, 2 R)-2-[(4-m e t h o x y b e n z y l o x y)$ methyl]cyclopentyl $\}-9,11,13,15$-tetramethyl-18oxooxacyclooctadeca-4,6-diene-7-carboxylate (48)

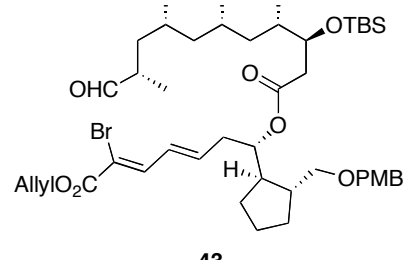

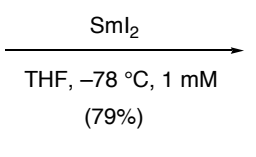

43

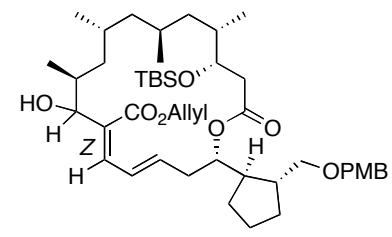

48

To a solution of $\mathrm{SmI}_{2}(0.1 \mathrm{M}$ in THF, $16.7 \mathrm{ml}, 1.67 \mathrm{mmol})$ was added dropwise a solution of $43(70.5 \mathrm{mg}, 83.0 \mu \mathrm{mol})$ in THF $(66 \mathrm{ml})$ over $1 \mathrm{~h}$ at $-78^{\circ} \mathrm{C}$ under an argon atmosphere. After exposure to oxgen gas, the reaction mixture was treated with silica gel $(21 \mathrm{~g})$ and hexane $(50 \mathrm{ml})$ and stirred for $30 \mathrm{~min}$ at room temperature. The resulting mixture was filtered through a pad of silica gel (eluate EtOAc) and concentrated in vacuo. The residue was purified by column chromatography (20:1 hexanes/EtOAc) to give 48 (50.9 mg, 79\%, C11 stereochemistry is unknown.) as a yellow oil and the cyclized diasteromixtures $(13.1 \mathrm{mg}, 21 \%)$.

$48:[\alpha]_{D}^{26}-28.0^{\circ}\left(c 0.18, \mathrm{CHCl}_{3}\right)$; IR (KBr) 2952, 2864, 2357, 1730, 1624, 1514, 1460, 1373, 1296, 1248, 1173, 1084, 833, $777 \mathrm{~cm}^{-1}$; ${ }^{1} \mathrm{H}-\mathrm{NMR}\left(300 \mathrm{MHz}, \mathrm{CDCl}_{3}\right) \delta 0.09$ (s, $3 \mathrm{H}), 0.14(\mathrm{~s}, 3 \mathrm{H}), 0.64(\mathrm{~m}, 1 \mathrm{H}), 0.73-0.92(\mathrm{~m}, 23 \mathrm{H}), 0.97-1.60(\mathrm{~m}, 9 \mathrm{H}), 1.66-2.08(\mathrm{~m}$, $6 \mathrm{H}), 2.14-2.55(\mathrm{~m}, 4 \mathrm{H}), 3.24(\mathrm{dd}, 1 \mathrm{H}, J=9.0,7.7 \mathrm{~Hz}), 3.38(\mathrm{dd}, 1 \mathrm{H}, J=9.0,5.7 \mathrm{~Hz})$, $3.79(\mathrm{~s}, 3 \mathrm{H}), 3.95(\mathrm{~m}, 1 \mathrm{H}), 4.39(\mathrm{~d}, 1 \mathrm{H}, J=11.7 \mathrm{~Hz}), 4.45(\mathrm{~d}, 1 \mathrm{H}, J=11.7 \mathrm{~Hz}), 4.48(\mathrm{~d}$, $1 \mathrm{H}, J=2.2 \mathrm{~Hz}), 4.65(\mathrm{ddt}, 1 \mathrm{H}, J=13.1,5.8,1.3 \mathrm{~Hz}), 4.72(\mathrm{ddt}, 1 \mathrm{H}, J=13.1,5.8,1.3$ $\mathrm{Hz}), 5.07(\mathrm{~m}, 1 \mathrm{H}), 5.25(\mathrm{dd}, 1 \mathrm{H}, J=10.4,1.3 \mathrm{~Hz}), 5.34(\mathrm{dd}, 1 \mathrm{H}, J=17.2,1.3 \mathrm{~Hz}), 5.83$ $(\mathrm{m}, 1 \mathrm{H}), 5.96(\mathrm{ddt}, 1 \mathrm{H}, J=17.2,10.4,5.8 \mathrm{~Hz}), 6.58(\mathrm{~d}, 1 \mathrm{H}, J=11.4 \mathrm{~Hz}), 6.86(\mathrm{~d}, 2 \mathrm{H}, J$ $=8.5 \mathrm{~Hz}), 7.08(\mathrm{dd}, 1 \mathrm{H}, J=15.0,11.4 \mathrm{~Hz}), 7.25(\mathrm{~d}, 2 \mathrm{H}, J=8.5 \mathrm{~Hz}) ;{ }^{13} \mathrm{C}-\mathrm{NMR}(100$ $\left.\mathrm{MHz}, \mathrm{CDCl}_{3}\right) \delta-4.6,-4.1,14.8,17.6,18.0,18.4,20.0,25.0,25.8,25.9,26.2,29.1$, 30.2 , 34.2, 34.7, 35.2, 35.8, 37.8, 41.7, 43.3, 46.4, 48.5, 55.2, 65.0, 72.3, 72.7, 74.3, 75.7, 76.7, 113.7, 118.5, 129.0, 129.4, 130.7, 132.1, 139.0, 139.3, 140.9, 159.0, 166.6, 171.5; HRMS (FAB, $m$-NBA+NaI) $[\mathrm{M}+\mathrm{Na}]^{+}$calcd for $\mathrm{C}_{45} \mathrm{H}_{72} \mathrm{O}_{8} \mathrm{SiNa} 791.4894$, found 791.4894 .

$(2 S, 4 E, 8 S, 9 S, 11 R, 13 S, 15 S, 16 S)$-Allyl 16-(tert-butyldimethylsilyloxy)-8-hydroxy-2$\{(1 R, 2 R)-2-[(4-m e t h o x y b e n z y l o x y) m e t h y l] c y c l o p e n t y l\}-9,11,13,15-t e t r a m e t h y l-18-$ oxooxacyclooctadeca-4,6-diene-7-carboxylate (51)

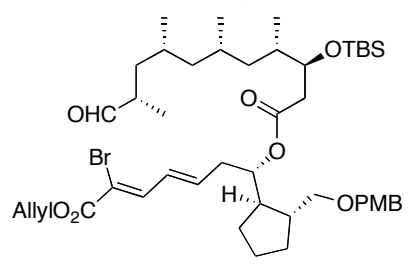

43

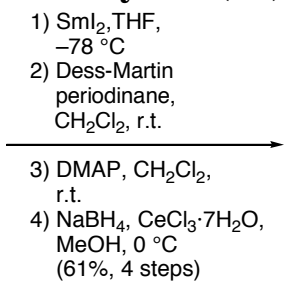

(61\%, 4 steps)

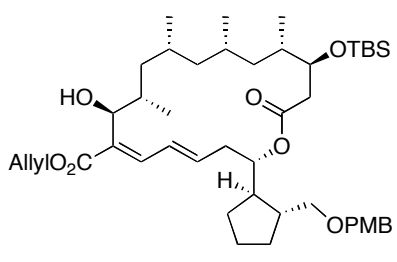

51

To a solution of $\mathrm{SmI}_{2}(0.1 \mathrm{M}$ in THF, $13.3 \mathrm{ml}, 1.33 \mathrm{mmol})$ was added dropwise a solution of $43(56.3 \mathrm{mg}, 66.5 \mu \mathrm{mol})$ in THF $(53.3 \mathrm{ml})$ over $1 \mathrm{~h}$ at $-78^{\circ} \mathrm{C}$ under an argon atmosphere. After exposure to oxgen gas, the reaction mixture was treated with silica gel $(16 \mathrm{~g})$ and hexane $(28 \mathrm{ml})$ and stirred for $30 \mathrm{~min}$ at room temperature. The resulting mixture was filtered through a pad of silica gel (eluate EtOAc) and concentrated in 
vacuo. The residue was briefly purified by column chromatography $(20: 1$ hexanes/EtOAc) to give the cyclized diasteromixtures.

To a solution of the mixture $(51.1 \mathrm{mg}, 0.067 \mathrm{mmol})$ in $\mathrm{CH}_{2} \mathrm{Cl}_{2}(1.3 \mathrm{ml})$ was added DessMartin periodinane $(70.5 \mathrm{mg}, 0.166 \mathrm{mmol})$ at room teperature. After stirring for $0.5 \mathrm{~h}$, the reaction mixture was quenched with aqueous $\mathrm{NaHCO}_{3}$ solution and aqueous $\mathrm{Na}_{2} \mathrm{~S}_{2} \mathrm{O}_{3}$ solution and extracted with $\mathrm{CH}_{2} \mathrm{Cl}_{2}$. The combined organic phases were dried over anhydrous $\mathrm{Na}_{2} \mathrm{SO}_{4}$ and concentrated in vacuo to afford the crude ketones 49 and 50.

To a solution of the crude ketones in $\mathrm{CH}_{2} \mathrm{Cl}_{2}(1.3 \mathrm{ml})$ was added DMAP (12.3 mg, 0.099 mmol). After 7 day, the reaction mixture was diluted with water and extracted with $\mathrm{CH}_{2} \mathrm{Cl}_{2}$. The combined organic phases were dried over anhydrous $\mathrm{Na}_{2} \mathrm{SO}_{4}$ and concentrated in vacuo to give the crude ketones 49 and $\mathbf{5 0 .}$

To a solution of the mixture in $\mathrm{MeOH}(1.3 \mathrm{ml})$ was added $\mathrm{CeCl}_{3} \cdot 7 \mathrm{H}_{2} \mathrm{O}(37.2 \mathrm{mg}, 0.100$ mmol) and $\mathrm{NaBH}_{4}(3.80 \mathrm{mg}, 0.100 \mathrm{mmol})$ at $0^{\circ} \mathrm{C}$. After stirring for $0.5 \mathrm{~h}$, the reaction mixture was treated with water. After $\mathrm{MeOH}$ was removed in vacuo, the resulting mixture was extracted with EtOAc. The combined organic layers were dried over anhydrous $\mathrm{Na}_{2} \mathrm{SO}_{4}$ and removed in vacuo. The residue was purified by column chromatography (25: 1 hexanes/EtOAc) to give $\mathbf{5 1}$ (31.2 $\mathrm{mg}, 61 \%$ for 4 steps) as a yellow oil. $[\alpha]_{D}^{24}-21.6^{\circ}\left(c 0.17, \mathrm{CHCl}_{3}\right)$; IR (KBr) 2952, 2864, 1738, 1693, 1630, 1417 , 1373, 1246, 1171, 1082, 835, $775 \mathrm{~cm}^{-1}$; ${ }^{1} \mathrm{H}-\mathrm{NMR}\left(300 \mathrm{MHz}, \mathrm{CDCl}_{3}\right) \delta 0.07$ (s, 3H), $0.08(\mathrm{~s}, 3 \mathrm{H}), 0.72-0.90(\mathrm{~m}, 22 \mathrm{H}), 0.92-1.10(\mathrm{~m}, 3 \mathrm{H}), 1.17-1.61(\mathrm{~m}, 5 \mathrm{H}), 1.68-1.77(\mathrm{~m}$, $4 \mathrm{H}), 1.83-1.96(\mathrm{~m}, 2 \mathrm{H}), 1.97-2.16(\mathrm{~m}, 3 \mathrm{H}), 2.19-2.47(\mathrm{~m}, 4 \mathrm{H}), 3.24(\mathrm{dd}, 1 \mathrm{H}, J=8.8,7.7$ $\mathrm{Hz}), 3.37(\mathrm{dd}, 1 \mathrm{H}, J=8.8,5.7 \mathrm{~Hz}), 3.79(\mathrm{~s}, 3 \mathrm{H}), 3.99(\mathrm{~m}, 1 \mathrm{H}), 4.40(\mathrm{~d}, 1 \mathrm{H}, J=11.8$ $\mathrm{Hz}), 4.45(\mathrm{~d}, 1 \mathrm{H}, J=11.8 \mathrm{~Hz}), 4.61(\mathrm{ddt}, 1 \mathrm{H}, J=13.1,5.8,1.3 \mathrm{~Hz}), 4.67(\mathrm{ddt}, 1 \mathrm{H}, J=$ $13.1,5.8,1.30 \mathrm{~Hz}), 4.67(\mathrm{~m}, 1 \mathrm{H}), 5.02(\mathrm{~m}, 1 \mathrm{H}), 5.26(\mathrm{dd}, 1 \mathrm{H}, J=10.4,1.1 \mathrm{~Hz}), 5.34$ $(\mathrm{dd}, 1 \mathrm{H}, J=17.4,1.1 \mathrm{~Hz}), 5.89-6.03(\mathrm{~m}, 2 \mathrm{H}), 6.57(\mathrm{~m}, 1 \mathrm{H}), 6.86(\mathrm{~d}, 2 \mathrm{H}, J=8.5 \mathrm{~Hz})$, $7.23(\mathrm{~d}, 1 \mathrm{H}, J=11.2 \mathrm{~Hz}), 7.25(\mathrm{~d}, 2 \mathrm{H}, J=8.5 \mathrm{~Hz}) ;{ }^{13} \mathrm{C}-\mathrm{NMR}\left(100 \mathrm{MHz}, \mathrm{CDCl}_{3}\right) \delta-5.1$, -4.0, 13.8, 17.9, 18.8, 19.7, 25.0, 25.8, 26.0, 26.5, 29.5, 29.7, 30.2, 35.8, 36.2, 36.7, $37.8,41.8,43.7,45.8,48.4,55.2,65.5,72.7,73.3,74.3,74.8,75.2,113.7,119.9,128.0$, $129.1,130.7,132.0,139.3,141.2,141.7,159.0,169.0,171.5$; HRMS (FAB, m$\mathrm{NBA}+\mathrm{NaI})[\mathrm{M}+\mathrm{Na}]^{+}$calcd for $\mathrm{C}_{45} \mathrm{H}_{72} \mathrm{O}_{8} \mathrm{SiNa} 791.4894$, found 791.4914 .

$(2 S, 4 E, 6 E, 8 S, 9 S, 11 R, 13 S, 15 S, 16 S)$-Allyl 8,16-bis(tert-butyldimethylsilyloxy)-2$\{(1 R, 2 R)-2-[(4-m e t h o x y b e n z y l o x y) m e t h y l] c y c l o p e n t y l\}-9,11,13,15-t e t r a m e t h y l-18-$ oxooxacyclooctadeca-4,6-diene-7-carboxylate (53)
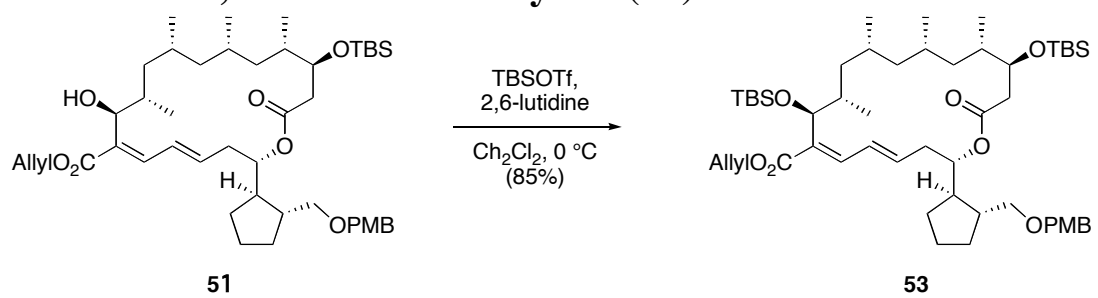

A solution of $51(29.8 \mathrm{mg}, 0.039 \mathrm{mmol})$ and 2,6-lutidine $(0.010 \mathrm{ml}, 0.066 \mathrm{mmol})$ in $\mathrm{CH}_{2} \mathrm{Cl}_{2}(0.8 \mathrm{ml})$ was cooled to $0{ }^{\circ} \mathrm{C}$ under an nitrogen atmosphere. To the stirring solution was added TBSOTf $(0.010 \mathrm{ml}, 0.050 \mathrm{mmol})$. After stirring for $30 \mathrm{~min}$, the mixture was treated with $\mathrm{H}_{2} \mathrm{O}$ and extracted with $\mathrm{CH}_{2} \mathrm{Cl}_{2}$. The combined organic phases were dried over anhydrous $\mathrm{Na}_{2} \mathrm{SO}_{4}$ and concentrated under reduced pressure. The 
residue was purified by column chromatography (60:1 hexanes/EtOAc) to give $\mathbf{5 3}$ (19.1 mg, 85\%) as a colorless oil. $[\alpha]_{\mathrm{D}}^{25}-9.2^{\circ}\left(c 0.17, \mathrm{CHCl}_{3}\right)$; IR (KBr) 2947, 2862, $2357,1736,1631,1514,1458,1371,1244,1169,1076,835,779,675 \mathrm{~cm}^{-1}$; ${ }^{1} \mathrm{H}-\mathrm{NMR}$ $\left(300 \mathrm{MHz}, \mathrm{CDCl}_{3}\right) \delta-0.09(\mathrm{~s}, 3 \mathrm{H}), 0.04(\mathrm{~s}, 3 \mathrm{H}), 0.05(\mathrm{~s}, 3 \mathrm{H}), 0.05$ (s, 3H), 0.71-0.90 $(\mathrm{m}, 30 \mathrm{H}), 0.95-1.22(\mathrm{~m}, 5 \mathrm{H}), 1.25-2.03(\mathrm{~m}, 13 \mathrm{H}), 2.27(\mathrm{~d}, 2 \mathrm{H}, J=6.5 \mathrm{~Hz}), 2.42(\mathrm{~m}$, $2 \mathrm{H}), 3.18(\mathrm{t}, 1 \mathrm{H}, J=8.9 \mathrm{~Hz}), 3.38(\mathrm{dd}, 1 \mathrm{H}, J=8.9,4.4 \mathrm{~Hz}), 3.78(\mathrm{~s}, 3 \mathrm{H}), 3.93(\mathrm{~m}, 1 \mathrm{H})$, $4.41(\mathrm{~s}, 2 \mathrm{H}), 4.63(\mathrm{ddt}, 1 \mathrm{H}, J=13.1,5.8,1.3 \mathrm{~Hz}), 4.67$ (ddt, $1 \mathrm{H}, J=13.1,5.8,1.3 \mathrm{~Hz}$ ), $4.80(\mathrm{~m}, 1 \mathrm{H}), 4.95(\mathrm{~m}, 1 \mathrm{H}), 5.23(\mathrm{dd}, 1 \mathrm{H}, J=10.4,1.4 \mathrm{~Hz}), 5.32(\mathrm{dd}, J=17.2,1.4 \mathrm{~Hz})$, 5.89-6.09 (m, 2H), $6.66(\mathrm{~d}, 1 \mathrm{H}, J=11.2 \mathrm{~Hz}), 6.86(\mathrm{~d}, 2 \mathrm{H}, J=8.7 \mathrm{~Hz}), 7.08(\mathrm{~m}, 1 \mathrm{H})$, $7.24(\mathrm{~d}, 2 \mathrm{H}, J=8.7 \mathrm{~Hz}$ ); HRMS (FAB, $m-\mathrm{NBA}+\mathrm{NaI})[\mathrm{M}+\mathrm{Na}]^{+}$calcd for $\mathrm{C}_{51} \mathrm{H}_{86} \mathrm{O}_{8} \mathrm{Si}_{2} \mathrm{Na}$ 905.5759, found 905.5740 .

$(2 S, 4 E, 6 E, 8 S, 9 S, 11 R, 13 S, 15 S, 16 S)$-16-(tert-Butyldimethylsilyloxy)-8-hydroxy-2$\{(1 R, 2 R)-2-[(4-m e t h o x y b e n z y l o x y) m e t h y l] c y c l o p e n t y l\}-9,11,13,15-t e t r a m e t h y l-18-$ oxooxacyclooctadeca-4,6-diene-7-carboxylic acid (55)

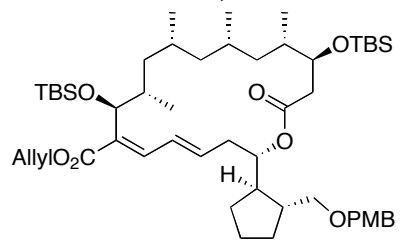

53

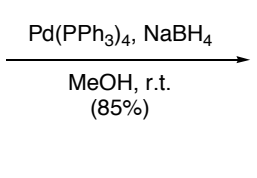

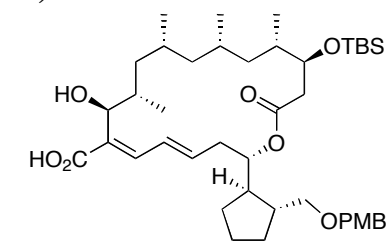

55

To a solution of $53(9.50 \mathrm{mg}, 0.011 \mathrm{mmol})$ in $\mathrm{MeOH}(0.22 \mathrm{ml})$ at $0^{\circ} \mathrm{C}$ were added $\mathrm{Pd}\left(\mathrm{PPh}_{3}\right)_{4}(1.00 \mathrm{mg}, 0.001 \mathrm{mmol})$ and $\mathrm{NaBH}_{4}(1.20 \mathrm{mg}, 0.032 \mathrm{mmol})$. After stirring for $30 \mathrm{~min}$ at $0{ }^{\circ} \mathrm{C}$, the reaction mixture was diluted with water and $\mathrm{MeOH}$ was concentrated under reduced pressure. The resulting mixture was extracted with EtOAc. The combined organic phases were dried over anhydrous $\mathrm{Na}_{2} \mathrm{SO}_{4}$ and concentrated in vacuo. The residue was purified by column chromatography $(2: 1$ hexanes/EtOAc) to give $55(6.7 \mathrm{mg}, 85 \%)$ as a colorless oil. $[\alpha]_{\mathrm{D}}^{27}-34.4^{\circ}\left(c 0.17, \mathrm{CHCl}_{3}\right) ;{ }^{1} \mathrm{H}-\mathrm{NMR}$ $\left(300 \mathrm{MHz}, \mathrm{CDCl}_{3}\right) \delta 0.07(\mathrm{~s}, 3 \mathrm{H}), 0.09(\mathrm{~s}, 3 \mathrm{H}), 0.75-1.18(\mathrm{~m}, 25 \mathrm{H}), 1.20-1.77(\mathrm{~m}, 9 \mathrm{H})$, $1.87-2.45(\mathrm{~m}, 9 \mathrm{H}), 3.24(\mathrm{dd}, 1 \mathrm{H}, J=9.0,7.9 \mathrm{~Hz}), 3.37(\mathrm{dd}, 1 \mathrm{H}, J=9.0,5.7 \mathrm{~Hz}), 3.79(\mathrm{~s}$, $3 \mathrm{H}), 3.99(\mathrm{~m}, 1 \mathrm{H}), 4.40(\mathrm{~d}, 1 \mathrm{H}, J=11.7 \mathrm{~Hz}), 4.45(\mathrm{~d}, 1 \mathrm{H}, J=11.7 \mathrm{~Hz}), 4.68(\mathrm{~d}, 1 \mathrm{H}, J=$ $3.6 \mathrm{~Hz}), 5.02(\mathrm{~m}, 1 \mathrm{H}), 6.02(\mathrm{~m}, 1 \mathrm{H}), 6.59(\mathrm{~m}, 1 \mathrm{H}), 6.86(\mathrm{~d}, 2 \mathrm{H}, J=8.6 \mathrm{~Hz}), 7.25(\mathrm{~d}, 2 \mathrm{H}$, $J=8.6 \mathrm{~Hz}), 7.34(\mathrm{~d}, 1 \mathrm{H}, J=11.5 \mathrm{~Hz}) ;{ }^{13} \mathrm{C}-\mathrm{NMR}\left(100 \mathrm{MHz}, \mathrm{CDCl}_{3}\right) \delta-5.1,-4.0,14.1$, $17.9,18.8,19.6,21.0,22.7,25.0,25.8,26.2,26.5,29.5,30.2,33.6,35.8,36.4,36.7$, 41.9, 43.7, 45.7, 48.3, 55.2, 72.7, 73.4, 74.3, 74.6, 75.2, 113.8, 125.8, 128.1, 129.1, $130.7,142.3,143.7,159.1,171.5,172.1$; LRMS (FAB, $m-\mathrm{NBA}+\mathrm{NaI})[\mathrm{M}-\mathrm{H}]^{+} 727$, $[\mathrm{M}+\mathrm{Na}]^{+} 751$. 
$(2 S, 4 E, 6 E, 8 S, 9 S, 11 R, 13 S, 15 S, 16 S)$-16-(tert-Butyldimethylsilyloxy)-8-hydroxy-2$\{(1 R, 2 R)-2-[(4-m e t h o x y b e n z y l o x y) m e t h y l] c y c l o p e n t y l\}-9,11,13,15-t e t r a m e t h y l-18-$ oxooxacyclooctadeca-4,6-diene-7-carboxamide (56)

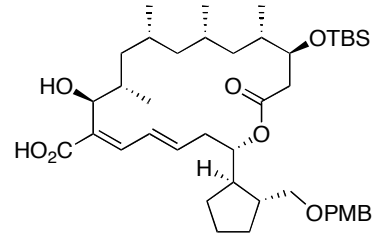

55
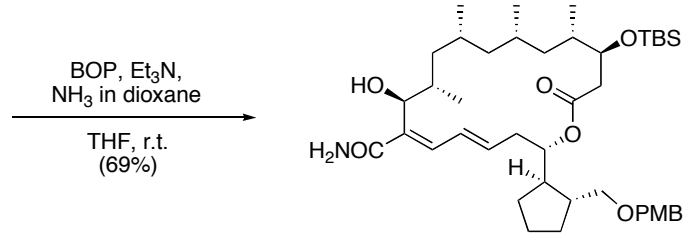

56

To a solution of $55(20.3 \mathrm{mg}, 0.028 \mathrm{mmol})$ in THF $(0.56 \mathrm{ml})$ at room temperature were added a solution of $\mathrm{NH}_{3}$ in dioxane $(0.5 \mathrm{M}, 0.560 \mathrm{ml}), \mathrm{Et}_{3} \mathrm{~N}(0.020 \mathrm{ml}, 0.112 \mathrm{mmol})$ and BOP $(24.7 \mathrm{mg}, 0.056 \mathrm{mmol})$. The reaction mixture was stirred for $21.5 \mathrm{~h}$ at $23{ }^{\circ} \mathrm{C}$ and then treated with a solution of $\mathrm{NH}_{3}$ in dioxane $(0.5 \mathrm{M}, 0.560 \mathrm{ml})$ again. After stirring for $26.5 \mathrm{~h}$ (total $48 \mathrm{~h}$ ), the reaction mixture was quenched with $\mathrm{NH}_{4} \mathrm{Cl}$ solution and extracted with EtOAc. The combined organic layers was dried over anhydrous $\mathrm{Na}_{2} \mathrm{SO}_{4}$ and removed in vacuo. The residue was purified by column chromatography (1: 1 hexanes/EtOAc) to give $56(13.9 \mathrm{mg}, 69 \%)$ as a colorless oil. ${ }^{1} \mathrm{H}-\mathrm{NMR}(300 \mathrm{MHz}$, $\left.\mathrm{CDCl}_{3}\right) \delta 0.09(\mathrm{~s}, 3 \mathrm{H}), 0.11(\mathrm{~s}, 3 \mathrm{H}), 0.76-0.87(\mathrm{~m}, 21 \mathrm{H}), 0.96-2.14(\mathrm{~m}, 18 \mathrm{H}), 2.22-2.48$ $(\mathrm{m}, 4 \mathrm{H}), 3.27(\mathrm{dd}, 1 \mathrm{H}, J=8.5,7.3 \mathrm{~Hz}), 3.37(\mathrm{dd}, 1 \mathrm{H}, J=8.5,6.4 \mathrm{~Hz}), 3.80(\mathrm{~s}, 3 \mathrm{H}), 4.04$ $(\mathrm{m}, 1 \mathrm{H}), 4.40(\mathrm{~d}, 1 \mathrm{H}, J=11.7 \mathrm{~Hz}), 4.45(\mathrm{~d}, 1 \mathrm{H}, J=11.7 \mathrm{~Hz}), 4.70(\mathrm{~d}, 1 \mathrm{H}, J=3.0 \mathrm{~Hz})$, $5.00(\mathrm{~m}, 1 \mathrm{H}), 5.86(\mathrm{~m}, 1 \mathrm{H}), 6.44(\mathrm{dd}, 1 \mathrm{H}, J=14.2,11.2 \mathrm{~Hz}), 6.76(\mathrm{~d}, 1 \mathrm{H}, J=11.2 \mathrm{~Hz})$, $6.87(\mathrm{~d}, 2 \mathrm{H}, J=8.6 \mathrm{~Hz}), 7.25(\mathrm{~d}, 2 \mathrm{H}, J=8.6 \mathrm{~Hz})$

$(2 S, 4 E, 6 Z, 8 S, 9 S, 11 R, 13 S, 15 S, 16 S)$-16-(tert-Butyldimethylsilyloxy)-8-hydroxy-2$\{(1 R, 2 R)-2-[(4-m e t h o x y b e n z y l o x y) m e t h y l] c y c l o p e n t y l\}-9,11,13,15-t e t r a m e t h y l-18-$ oxooxacyclooctadeca-4,6-diene-7-carbonitrile (38)

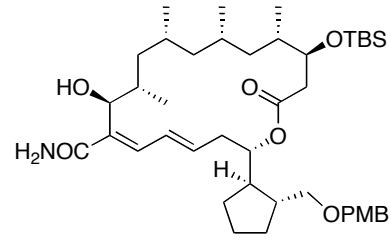

56

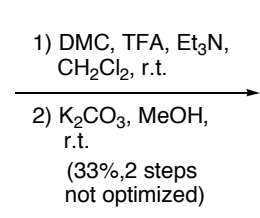

(33\%,2 steps
not optimized)

38

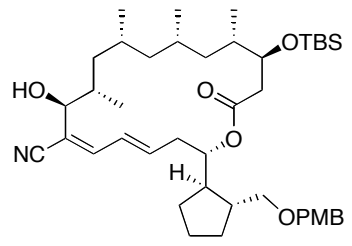

38

To a solution of $56(13.3 \mathrm{mg}, 0.018 \mathrm{mmol})$ in $\mathrm{CH}_{2} \mathrm{Cl}_{2}(0.4 \mathrm{ml})$ were added $\mathrm{Et}_{3} \mathrm{~N}(0.020$ $\mathrm{ml}, 0.132 \mathrm{mmol})$, TFA $(0.005 \mathrm{ml}, 0.066 \mathrm{mmol})$, and a solution of 2-chloro-1,3dimethylimidazolinium chloride (DMC) in $\mathrm{CH}_{2} \mathrm{Cl}_{2}(25 \%, 0.050 \mathrm{ml}, 0.066 \mathrm{mmol})$ under an argon atmosphere. After stirring for $1.5 \mathrm{~h}$, the reaction mixture was diluted with water and extracted with $\mathrm{CH}_{2} \mathrm{Cl}_{2}$. The combined organic phases were dried over anhydrous $\mathrm{Na}_{2} \mathrm{SO}_{4}$ and concentrated in vacuo. The crude product was purified by column chromatography (15:1 hexanes/EtOAc, EtOAc) to give the corresponding C8trifluoroacetyl compound (4.60 mg, $0.006 \mathrm{mmol}, 31 \%)$ and $38(1.30 \mathrm{mg}, 0.002 \mathrm{mmol}$, $10 \%)$.

To a solution of the C8-trifluoroacetyl compound in $\mathrm{MeOH}(0.5 \mathrm{ml})$ at room temperature was added $\mathrm{K}_{2} \mathrm{CO}_{3}(3.60 \mathrm{mg}, 0.026 \mathrm{mmol})$. After stirring for $1.5 \mathrm{~h}$, the mixture was treated with an additional $\mathrm{K}_{2} \mathrm{CO}_{3}(3.60 \mathrm{mg}, 0.026 \mathrm{mmol})$ and stirred for 2.5 $\mathrm{h}$ at room temperature. The reaction mixture was then stirred for $1.0 \mathrm{~h}$ at $50^{\circ} \mathrm{C}$ and quenced with $\mathrm{H}_{2} \mathrm{O}$. After $\mathrm{MeOH}$ was removed in vacuo, the resulting mixture was extracted with EtOAc. The combined organic layers was dried over anhydrous $\mathrm{Na}_{2} \mathrm{SO}_{4}$ 
and removed in vacuo. The crude product was purified by column chromatography ( 5 : 1 hexanes/EtOAc) to give $\mathbf{3 8}$ (2.96 mg, 73\%, total $4.26 \mathrm{mg}, 33 \%$ for 2 steps).

$(S, 2 Z, 4 E)$ - E th y l 2 - b romo-7-(tert-butyldimethylsilyloxy)-7-\{(1R,2R)-2-[(4methoxybenzyloxy)methyl]cyclopentyl $\}$ hepta-2,4-dienoate (29a)

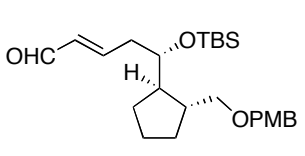

27

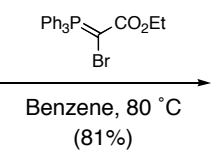

$(81 \%)$

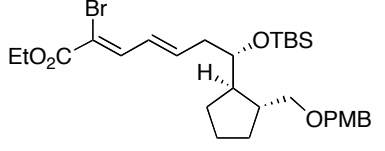

29a

A solution of 27 (184 mg, $0.426 \mathrm{mmol})$ in benzene $(4.2 \mathrm{ml})$ was treated with (ethoxycarbonylbromomethylidene)triphenylphosphorane (544 mg, $1.27 \mathrm{mmol}$ ) and refluxed at $80{ }^{\circ} \mathrm{C}$ for $12 \mathrm{~h}$. The reaction mixture was concentrated in vacuo and purified by column chromatography (40:1 hexanes/EtOAc) to give 29a (201 $\mathrm{mg}, 81 \%)$ as a colorless oil. ${ }^{1} \mathrm{H}-\mathrm{NMR}\left(270 \mathrm{MHz}, \mathrm{CDCl}_{3}\right) \delta 0.04(\mathrm{~s}, 3 \mathrm{H}), 0.05(\mathrm{~s}, 3 \mathrm{H}), 0.88(\mathrm{~s}, 9 \mathrm{H}), 1.26$ $(\mathrm{m}, 1 \mathrm{H}), 1.34(\mathrm{t}, 3 \mathrm{H}, J=7.1 \mathrm{~Hz}), 1.46-1.72(\mathrm{~m}, 6 \mathrm{H}), 2.11(\mathrm{~m}, 1 \mathrm{H}), 2.39(\mathrm{~m}, 2 \mathrm{H}), 3.18(\mathrm{t}$, $1 \mathrm{H}, J=8.6 \mathrm{~Hz}), 3.41(\mathrm{dd}, 1 \mathrm{H}, J=8.6,5.8 \mathrm{~Hz}), 3.70(\mathrm{~m}, 1 \mathrm{H}), 3.80(\mathrm{~s}, 3 \mathrm{H}), 4.29(\mathrm{q}, 2 \mathrm{H}$, $J=7.1 \mathrm{~Hz}), 4.39(\mathrm{~d}, 1 \mathrm{H}, J=11.9 \mathrm{~Hz}), 4.45(\mathrm{~d}, 1 \mathrm{H}, J=11.9 \mathrm{~Hz}), 6.34(\mathrm{~m}, 1 \mathrm{H}), 6.48(\mathrm{dd}$, $1 \mathrm{H}, J=15.5,10.2 \mathrm{~Hz}), 6.86(\mathrm{~d}, 2 \mathrm{H}, J=8.6 \mathrm{~Hz}), 7.24(\mathrm{~d}, 2 \mathrm{H}, J=8.6 \mathrm{~Hz}), 7.62(\mathrm{~d}, 1 \mathrm{H}, J$ $=10.2 \mathrm{~Hz}) ;{ }^{13} \mathrm{C}-\mathrm{NMR}\left(67.5 \mathrm{MHz}, \mathrm{CDCl}_{3}\right) \delta-4.6,-4.2,14.2,18.0,25.3,25.9,29.4$, 30.4, 39.3, 41.3, 47.4, 55.2, 62.3, 72.7, 74.6, 75.1, 113.7, 129.1, 129.4, 130.8, 141.2, $144.1,159.0,163.1,190.8$; HRMS $(\mathrm{FAB}, m-\mathrm{NBA}+\mathrm{NaI})[\mathrm{M}+\mathrm{Na}]^{+}$calcd for $\mathrm{C}_{29} \mathrm{H}_{45} \mathrm{O}_{5} \mathrm{BrSiNa}$ 603.2117, found 603.2146.

$(S, 2 Z, 4 E)$-Ethyl 7-(tert-butyldimethylsilyloxy)-2-iodo-7-\{(1R,2R)-2-[(4methoxybenzyloxy)methyl]cyclopentyl\}hepta-2,4-dienoate (29b)

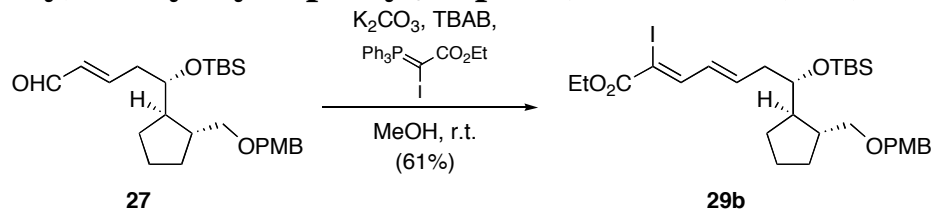

To a solution of 27 (205 $\mathrm{mg}, 0.572 \mathrm{mmol})$ in $\mathrm{MeOH}$ at room temperature were added (ethoxycarbonylbromomethylidene)triphenylphosphorane (568 $\mathrm{mg}, 0.944 \mathrm{mmol}$ ), TBAB (15.2 mg, $0.047 \mathrm{mmol})$, and $\mathrm{K}_{2} \mathrm{CO}_{3}(65.3 \mathrm{mg}, 0.472 \mathrm{mmol})$. The reaction mixture was then stirred at $50{ }^{\circ} \mathrm{C}$ for $9.5 \mathrm{~h}$ and concentrated in vacuo. The crude product was purified by column chromatography ( $80: 1$ hexanes/EtOAc) to give 29b $(180 \mathrm{mg}, 61 \%)$ as a colorless oil. $[\alpha]_{\mathrm{D}}^{24}=+19.4^{\circ}\left(c=0.50, \mathrm{CHCl}_{3}\right)$; IR $(\mathrm{KBr}) 2945$, $2858,1712,1622,1579,1514,1462,1363,1248,1180,1083,833,771 \mathrm{~cm}^{-1} ;{ }^{1} \mathrm{H}-\mathrm{NMR}$ $\left(300 \mathrm{MHz}, \mathrm{CDCl}_{3}\right) \delta 0.05(\mathrm{~s}, 3 \mathrm{H}), 0.05(\mathrm{~s}, 3 \mathrm{H}), 0.88(\mathrm{~s}, 9 \mathrm{H}), 1.34(\mathrm{t}, 3 \mathrm{H}, J=7.2 \mathrm{~Hz})$, 1.41-1.73 (m, 7H), $2.10(\mathrm{~m}, 1 \mathrm{H}), 2.32-2.48(\mathrm{~m}, 2 \mathrm{H}), 3.18(\mathrm{t}, 1 \mathrm{H}, J=8.7 \mathrm{~Hz}), 3.42(\mathrm{dd}$, $1 \mathrm{H}, J=8.7,5.3 \mathrm{~Hz}), 3.70(\mathrm{~m}, 1 \mathrm{H}), 3.79(\mathrm{~s}, 3 \mathrm{H}), 4.28(\mathrm{q}, 2 \mathrm{H}, J=7.2 \mathrm{~Hz}), 4.39(\mathrm{~d}, 1 \mathrm{H}, J$ $=11.6 \mathrm{~Hz}), 4.45(\mathrm{~d}, 1 \mathrm{H}, J=11.6 \mathrm{~Hz}), 6.35-6.46(\mathrm{~m}, 2 \mathrm{H}), 6.86(\mathrm{~d}, 2 \mathrm{H}, J=8.5 \mathrm{~Hz}), 7.25$ $(\mathrm{d}, 2 \mathrm{H}, J=8.5 \mathrm{~Hz}), 7.55(\mathrm{~d}, 1 \mathrm{H}, J=9.4 \mathrm{~Hz}) ;{ }^{13} \mathrm{C}-\mathrm{NMR}\left(100 \mathrm{MHz}, \mathrm{CDCl}_{3}\right) \delta-4.6,-4.2$, 14.2, 18.0, 25.3, 25.9, 29.4, 30.4, 39.3, 41.3, 47.4, 55.2, 62.6, 72.7, 74.6, 75.1, 90.5, $113.7,129.2$, 130.8, 134.0, 144.8, 147.6, 159.0, 163.6; HRMS (FAB, $m$-NBA+NaI) $[\mathrm{M}+\mathrm{Na}]^{+}$calcd for $\mathrm{C}_{29} \mathrm{H}_{45} \mathrm{IO}_{5} \mathrm{SiNa} 651.1979$, found 651.1985.

$(S, 2 Z, 4 E)-2-B r o m o-7-(t e r t-b u t y l d i m e t h y l s i l y l o x y)-7-\{(1 R, 2 R)-2-[(4-$ 
methoxybenzyloxy)methyl]cyclopentyl\}hepta-2,4-dien-1-ol (59a)

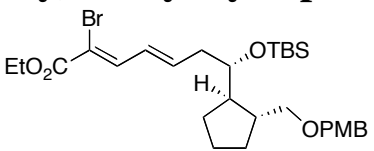

29a
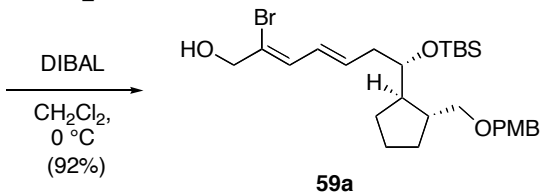

$59 a$

To a solution of 29a (101 $\mathrm{mg}, 0.171 \mathrm{mmol})$ in $\mathrm{CH}_{2} \mathrm{Cl}_{2}(1.7 \mathrm{ml})$ was added dropwise DIBAL $(0.94 \mathrm{M}$ in hexane, $0.460 \mathrm{ml}, 0.428 \mathrm{mmol})$ at $0^{\circ} \mathrm{C}$. After stirring for $0.5 \mathrm{~h}$, the reaction mixture was quenched with $\mathrm{MeOH}$, diluted with $\mathrm{CH}_{2} \mathrm{Cl}_{2}$, and treated with Celite $(0.2 \mathrm{~g})$ and $\mathrm{Na}_{2} \mathrm{SO}_{4} \cdot 10 \mathrm{H}_{2} \mathrm{O}(0.2 \mathrm{~g})$. The resulting mixture was stirred for $2 \mathrm{~h}$ at room temperature, filtered through a pad of Celite and concentrated in vacuo. The residue was purified by column chromatography $(15: 1$ hexanes/EtOAc) to give 59a (85.0 mg, 92\%) as a colorless oil. ${ }^{1} \mathrm{H}-\mathrm{NMR}\left(270 \mathrm{MHz}, \mathrm{CDCl}_{3}\right) \delta 0.04(\mathrm{~s}, 3 \mathrm{H}), 0.07$ (s, $3 \mathrm{H}), 0.88(\mathrm{~s}, 9 \mathrm{H}), 1.23-1.72(\mathrm{~m}, 7 \mathrm{H}), 2.12(\mathrm{~m}, 1 \mathrm{H}), 2.32(\mathrm{~m}, 2 \mathrm{H}), 3.18(\mathrm{t}, 1 \mathrm{H}, J=8.7$ $\mathrm{Hz}), 3.42(\mathrm{dd}, 1 \mathrm{H}, J=8.7,5.1 \mathrm{~Hz}), 3.64(\mathrm{~m}, 1 \mathrm{H}), 3.80(\mathrm{~s}, 3 \mathrm{H}), 4.29(\mathrm{~s}, 2 \mathrm{H}), 4.39(\mathrm{~d}, 1 \mathrm{H}$, $J=11.5 \mathrm{~Hz}), 4.45(\mathrm{~d}, 1 \mathrm{H}, J=11.5 \mathrm{~Hz}), 5.94(\mathrm{~m}, 1 \mathrm{H}), 6.30(\mathrm{dd}, 1 \mathrm{H}, J=15.2,9.9 \mathrm{~Hz})$, $6.51(\mathrm{~d}, 1 \mathrm{H}, J=9.9 \mathrm{~Hz}), 6.87(\mathrm{~d}, 2 \mathrm{H}, J=8.6 \mathrm{~Hz}), 7.25(\mathrm{~d}, 2 \mathrm{H}, J=8.6 \mathrm{~Hz})$; LRMS $(\mathrm{FAB}, m-\mathrm{NBA}+\mathrm{NaI})[\mathrm{M}+\mathrm{Na}]^{+} 561$.

$(S, 2 Z, 4 E)-7-($ tert-Butyldimethylsilyloxy)-2-iodo-7-\{(1R,2R)-2-[(4methoxybenzyloxy)methyl]cyclopentyl\}hepta-2,4-dien-1-ol (59b)

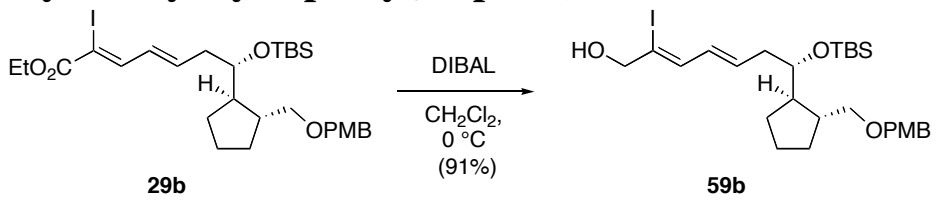

To a solution of $29 \mathbf{b}(291 \mathrm{mg}, 0.463 \mathrm{mmol})$ in $\mathrm{CH}_{2} \mathrm{Cl}_{2}(4.6 \mathrm{ml})$ was added dropwise DIBAL $(0.94 \mathrm{M}$ in hexane, $1.20 \mathrm{ml}, 1.16 \mathrm{mmol})$ at $0^{\circ} \mathrm{C}$. After stirring for $0.5 \mathrm{~h}$, the reaction mixture was quenched with $\mathrm{MeOH}$, diluted with $\mathrm{CH}_{2} \mathrm{Cl}_{2}$, and treated with Celite $(0.7 \mathrm{~g})$ and $\mathrm{Na}_{2} \mathrm{SO}_{4} \cdot 10 \mathrm{H}_{2} \mathrm{O}(0.7 \mathrm{~g})$. The mixture was stirred for $2 \mathrm{~h}$ at room temperature, filtered through a pad of Celite, and concentrated in vacuo. The residue was purified by column chromatography (20:1 hexanes/EtOAc) to give 59b $(247 \mathrm{mg}$, 91\%). $[\alpha]_{D}^{24}+22.8^{\circ}\left(c 0.18, \mathrm{CHCl}_{3}\right)$; IR (KBr) 2943, 2858, 1614, 1514, 1462, 1250, 1076, 833, $773 \mathrm{~cm}^{-1}$; ${ }^{1} \mathrm{H}-\mathrm{NMR}\left(300 \mathrm{MHz}, \mathrm{CDCl}_{3}\right) \delta 0.03$ (s, 6H), 0.87 (s, 9H), 1.45-1.73 $(\mathrm{m}, 7 \mathrm{H}), 2.11(\mathrm{~m}, 1 \mathrm{H}), 2.32(\mathrm{~m}, 2 \mathrm{H}), 3.17(\mathrm{t}, 1 \mathrm{H}, J=8.7 \mathrm{~Hz}), 3.42(\mathrm{dd}, 1 \mathrm{H}, J=8.7,5.1$ $\mathrm{Hz}), 3.65$ (m, 1H), 3.79 (s, 3H), 4.29 (bs, 2H), 4.38 (d, 1H, $J=11.6 \mathrm{~Hz}), 4.45$ (d, 1H, $J$ $=11.6 \mathrm{~Hz}), 5.99(\mathrm{~m}, 1 \mathrm{H}), 6.17(\mathrm{dd}, 1 \mathrm{H}, J=15.2,9.8 \mathrm{~Hz}), 6.42(\mathrm{~d}, 1 \mathrm{H}, J=9.8 \mathrm{~Hz}), 6.86$ $(\mathrm{d}, 2 \mathrm{H}, J=8.7 \mathrm{~Hz}), 7.24(\mathrm{~d}, 2 \mathrm{H}, J=8.7 \mathrm{~Hz}) ;{ }^{13} \mathrm{C}-\mathrm{NMR}\left(75 \mathrm{MHz}, \mathrm{CDCl}_{3}\right) \delta-4.6,-4.1$, 18.1, 25.3, 25.9, 29.5, 30.5, 38.9, 41.1, 47.1, 55.3, 71.7, 72.7, 75.3, 76.6, 105.2, 113.7, $129.2,130.9,133.1,134.0,136.9,159.0$; HRMS (FAB, $m-\mathrm{NBA}+\mathrm{NaI})[\mathrm{M}+\mathrm{Na}]^{+}$calcd for $\mathrm{C}_{27} \mathrm{H}_{43} \mathrm{IO}_{4} \mathrm{SiNa} 609.1873$, found 609.1854 . 
$(S, 2 Z, 4 E)-2-B r o m o-7-\{(1 R, 2 R)-2-[(4-m e t h o x y b e n z y l o x y)$ methyl]cyclopentyl $\}$ hepta2,4-diene-1,7-diol (60a)
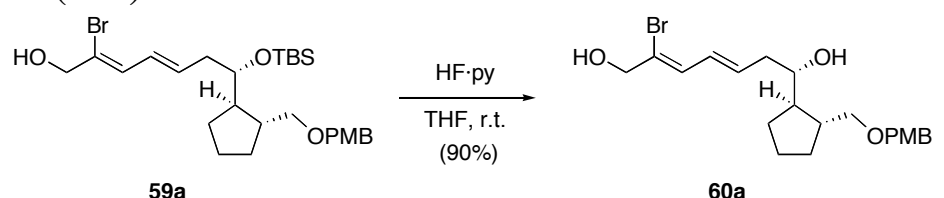

To a solution of 59a $(85.0 \mathrm{mg}, 0.158 \mathrm{mmol})$ in THF $(0.3 \mathrm{ml})$ at room temperature was added a solution of HF-pyridine in THF (HF-pyridine $:$ pyridine $:$ THF $=1: 1: 1,1.3$ $\mathrm{ml})$. After stirring for $16.5 \mathrm{~h}$ at room temperature, the reaction mixture was filtered through a pad of silica gel (eluate $\mathrm{CH}_{2} \mathrm{Cl}_{2}: \mathrm{MeOH}=60: 1$ ) and concentrated in vacuo. The residue was purified by column chromatography ( $2: 1$ hexanes/EtOAc) to give $\mathbf{6 0 a}$ (60.3 mg, 90\%) as a colorless oil. ${ }^{1} \mathrm{H}-\mathrm{NMR}\left(300 \mathrm{MHz}, \mathrm{CDCl}_{3}\right) \delta 1.17-1.29(\mathrm{~m}, 2 \mathrm{H})$, 1.39-1.65 (m, 3H), 1.69-1.84 (m, 2H), $2.05(\mathrm{~m}, 1 \mathrm{H}), 2.19(\mathrm{~m}, 1 \mathrm{H}),, 2.45(\mathrm{~m}, 1 \mathrm{H}), 3.15$ $(\mathrm{dd}, 1 \mathrm{H}, J=10.3,8.8 \mathrm{~Hz}), 3,54(\mathrm{dd}, 1 \mathrm{H}, J=8.8,4.1 \mathrm{~Hz}), 3.80(\mathrm{~s}, 3 \mathrm{H}), 4.73(\mathrm{~s}, 2 \mathrm{H}), 4.48$ $(\mathrm{s}, 2 \mathrm{H}), 6.11(\mathrm{~m}, 1 \mathrm{H}), 6.37(\mathrm{dd}, 1 \mathrm{H}, J=15.2,9.9 \mathrm{~Hz}), 6.57(\mathrm{~d}, 1 \mathrm{H}, J=9.9 \mathrm{~Hz}), 6.88(\mathrm{~d}$, $1 \mathrm{H}, J=8.6 \mathrm{~Hz}), 7.24(\mathrm{~d}, 1 \mathrm{H}, J=8.6 \mathrm{~Hz}) ; \mathrm{LRMS}(\mathrm{FAB}, m-\mathrm{NBA}+\mathrm{NaI})[\mathrm{M}+\mathrm{Na}]^{+} 447$.

$(S, 2 Z, 4 E)-2-I o d o-7-\{(1 R, 2 R)-2-[(4-m e t h o x y b e n z y l o x y)$ methyl $]$ cyclopentyl $\}$ hepta2,4-diene-1,7-diol (60b)

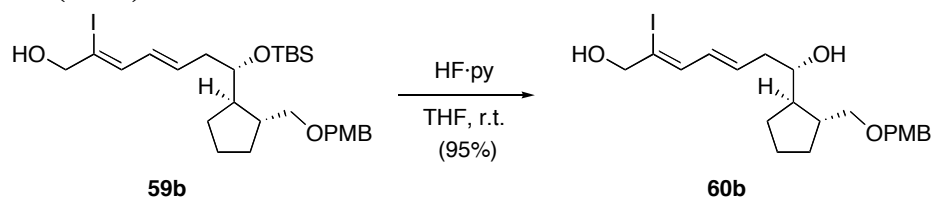

To a solution of $\mathbf{5 9 b}(135 \mathrm{mg}, 0.230 \mathrm{mmol})$ in THF $(0.4 \mathrm{ml})$ at room temperature was added a solution of HF-pyridine in THF (HF-pyridine : pyridine : THF $=1: 1: 1,2.0$ $\mathrm{ml}$ ). After stirring for $19 \mathrm{~h}$ at room temperature, the reaction mixture was filtered through a pad of silica gel (eluate $\mathrm{CH}_{2} \mathrm{Cl}_{2}: \mathrm{MeOH}=60: 1$ ) and concentrated in vacuo. The residue was purified by column chromatography ( $5: 1$ hexanes/EtOAc) to give $\mathbf{6 0 b}$ $(103 \mathrm{mg}, 95 \%)$ as a colorless oil. $[\alpha]_{\mathrm{D}}^{25}+22.6^{\circ}\left(c\right.$ 0.18, $\left.\mathrm{CHCl}_{3}\right)$; IR $(\mathrm{KBr}) 2943,2862$, 2359, 1612, 1514, 1452, 1248, 1080, 1036, $821 \mathrm{~cm}^{-1} ;{ }^{1} \mathrm{H}-\mathrm{NMR}\left(300 \mathrm{MHz}, \mathrm{CDCl}_{3}\right) \delta$ 1.17-1.29 (m, 2H), 1.39-1.64 (m, 3H), 1.69-1.84 (m, 2H), $2.05(\mathrm{~m}, 1 \mathrm{H}), 2.20(\mathrm{~m}, 1 \mathrm{H})$, $2.46(\mathrm{~m}, 1 \mathrm{H}), 3.14(\mathrm{dd}, 1 \mathrm{H}, J=10.4,8.7 \mathrm{~Hz}), 3.40(\mathrm{~m}, 1 \mathrm{H}), 3.54(\mathrm{dd}, 1 \mathrm{H}, J=8.7,4.1$ $\mathrm{Hz}), 3.80$ (s, 3H), 4.29 (s, 2H), 4.48 (s, 2H), 6.12-6.27 (m, 2H), 6.48 (d, 1H, J = 8.8 Hz), $6.87(\mathrm{~d}, 2 \mathrm{H}, J=8.6 \mathrm{~Hz}), 7.24(\mathrm{~d}, 2 \mathrm{H}, J=8.6 \mathrm{~Hz}) ;{ }^{13} \mathrm{C}-\mathrm{NMR}\left(75 \mathrm{MHz}, \mathrm{CDCl}_{3}\right) \delta 24.4$, 29.8, 31.0, 39.4, 43.9, 51.8, 55.2, 71.5, 72.9, 74.1, 75.0, 113.8, 129.3, 129.5, 132.8, 133.7, 137.0, 159.2; HRMS (FAB, $m-\mathrm{NBA}+\mathrm{NaI}$ ) $[\mathrm{M}+\mathrm{Na}]^{+}$calcd for $\mathrm{C}_{21} \mathrm{H}_{29} \mathrm{IO}_{4} \mathrm{SiNa}$ 495.1008, found 495.1014 .

$(S, 3 E, 5 Z)-6-B r o m o-1-\{(1 R, 2 R)-2-[(4-m e t h o x y b e n z y l o x y) m e t h y l] c y c l o p e n t y l\}-7-$ (triisopropylsilyloxy)hepta-3,5-dien-1-ol (61a)

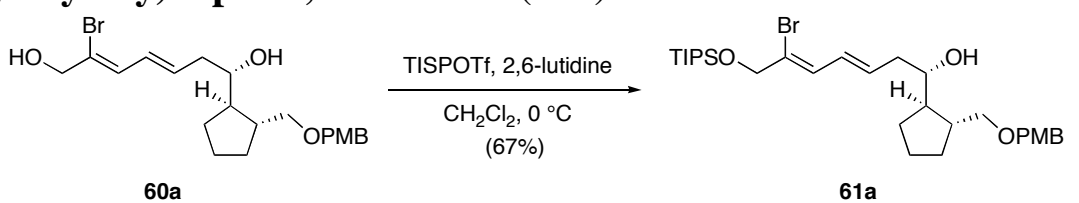

A solution of $\mathbf{6 0 a}(67.2 \mathrm{mg}, 0.158 \mathrm{mmol})$ in $\mathrm{CH}_{2} \mathrm{Cl}_{2}(1.6 \mathrm{ml})$ at $0{ }^{\circ} \mathrm{C}$ under nitrogen were treated with 2,6-lutidine $(24 \mu \mathrm{L}, 0.206 \mathrm{mmol})$ and TIPSOTf $(47 \mu \mathrm{L}, 0.174 \mathrm{mmol})$. 
After stirring for $1 \mathrm{~h}$ at $0{ }^{\circ} \mathrm{C}, 2,6$-lutidine $(24 \mu \mathrm{L}, 0.206 \mathrm{mmol})$ and TIPSOTf ( $47 \mu \mathrm{L}$, $0.174 \mathrm{mmol}$ ) were added again. The reaction mixture was stirred for $0.5 \mathrm{~h}$, diluted with $\mathrm{H}_{2} \mathrm{O}$, and extracted with $\mathrm{CH}_{2} \mathrm{Cl}_{2}$. The organic phases were dried over anhydrous $\mathrm{Na}_{2} \mathrm{SO}_{4}$ and concentrated in vacuo. The crude product was purified by column chromatography (40 : 1 hexanes/EtOAc) to give 61a $(61.6 \mathrm{mg}, 67 \%)$ as a colorless oil. ${ }^{1} \mathrm{H}-\mathrm{NMR}(300$ $\left.\mathrm{MHz}, \mathrm{CDCl}_{3}\right) \delta 1.04-1.30(\mathrm{~m}, 23 \mathrm{H}), 1.39-1.86(\mathrm{~m}, 5 \mathrm{H}), 2.05(\mathrm{~m}, 1 \mathrm{H}), 2.19(\mathrm{~m}, 1 \mathrm{H})$, $2.44(\mathrm{~m}, 1 \mathrm{H}), 3.16(\mathrm{dd}, 1 \mathrm{H}, J=10.2,8.7 \mathrm{~Hz}), 3,40(\mathrm{~m}, 1 \mathrm{H}), 3.52(\mathrm{dd}, 1 \mathrm{H}, J=8.7,4.3$ $\mathrm{Hz}), 3.80(\mathrm{~s}, 3 \mathrm{H}), 4.37(\mathrm{~s}, 2 \mathrm{H}), 4.40(\mathrm{~d}, 1 \mathrm{H}, J=11.7 \mathrm{~Hz}), 4.52(\mathrm{~d}, 1 \mathrm{H}, J=11.7 \mathrm{~Hz}), 6.07$ $(\mathrm{m}, 1 \mathrm{H}), 6.41(\mathrm{dd}, 1 \mathrm{H}, J=15.5,10.1 \mathrm{~Hz}), 6.69(\mathrm{~d}, 1 \mathrm{H}, J=10.1 \mathrm{~Hz}), 6.87(\mathrm{~d}, 2 \mathrm{H}, J=8.6$ $\mathrm{Hz}), 7.24(\mathrm{~d}, 2 \mathrm{H}, J=8.6 \mathrm{~Hz})$; HRMS $(\mathrm{FAB}, m-\mathrm{NBA}+\mathrm{NaI})[\mathrm{M}+\mathrm{Na}]^{+}$calcd for $\mathrm{C}_{30} \mathrm{H}_{49} \mathrm{BrO}_{4} \mathrm{SiNa}$ 603.2481, found 603.2462.

$(S, 3 E, 5 Z)-6-I o d o-1-\{(1 R, 2 R)-2-[(4-m e t h o x y b e n z y l o x y) m e t h y l] c y c l o p e n t y l\}-7-$ (triisopropylsilyloxy)hepta-3,5-dien-1-ol (61b)

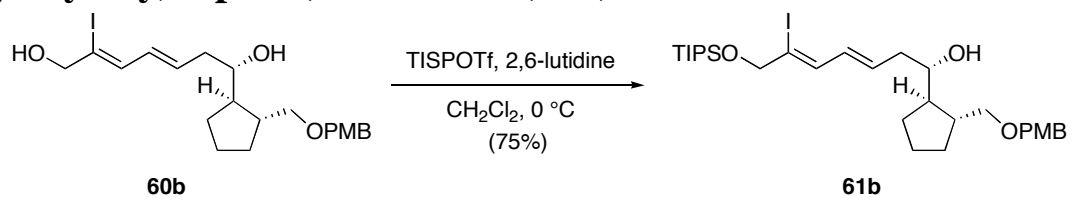

A solution of $60 \mathrm{~b}(16.5 \mathrm{mg}, 0.0350 \mathrm{mmol})$ in $\mathrm{CH}_{2} \mathrm{Cl}_{2}(0.4 \mathrm{ml})$ at $0{ }^{\circ} \mathrm{C}$ were treated with 2,6-lutidine $(20 \mu \mathrm{L}, 0.182 \mathrm{mmol})$ and TIPSOTf $(40 \mu \mathrm{L}, 0.153 \mathrm{mmol})$. After stirring for $6 \mathrm{~h}$ at $0{ }^{\circ} \mathrm{C}$, the reaction mixture was diluted with $\mathrm{H}_{2} \mathrm{O}$ and extracted with EtOAc. The organic phases were dried and concentrated in vacuo. The crude product was purified by column chromatography $\left(30: 1\right.$ hexanes/EtOAc) to give $\mathbf{6 1 b}(16.5 \mathrm{mg}, 75 \%) .[\alpha]_{\mathrm{D}}^{24}$ $+10.7^{\circ}\left(c 0.50, \mathrm{CHCl}_{3}\right)$; IR (KBr) 3427, 2945, 2864, 1614, 1514, 1460, 1248, 1115, 881, 809, $681 \mathrm{~cm}^{-1}$; ${ }^{1} \mathrm{H}-\mathrm{NMR}\left(300 \mathrm{MHz}, \mathrm{CDCl}_{3}\right) \delta 1.04-1.11(\mathrm{~m}, 21 \mathrm{H}), 1.25(\mathrm{~m}, 1 \mathrm{H}), 1.39-$ $1.67(\mathrm{~m}, 5 \mathrm{H}), 1.78(\mathrm{~m}, 1 \mathrm{H}), 2.06(\mathrm{~m}, 1 \mathrm{H}), 2.22(\mathrm{~m}, 1 \mathrm{H}), 2.45(\mathrm{~m}, 1 \mathrm{H}), 3.16(\mathrm{dd}, 1 \mathrm{H}, J=$ $10.2,8.8 \mathrm{~Hz}), 3.40(\mathrm{~m}, 1 \mathrm{H}), 3.52(\mathrm{dd}, 1 \mathrm{H}, J=8.8 \mathrm{~Hz}, 4.2 \mathrm{~Hz}), 3.79(\mathrm{~s}, 3 \mathrm{H}), 4.38(\mathrm{~s}$, $2 \mathrm{H}), 4.45(\mathrm{~d}, 1 \mathrm{H}, J=11.8 \mathrm{~Hz}), 4.51(\mathrm{~d}, 1 \mathrm{H}, J=11.8 \mathrm{~Hz}), 6.11(\mathrm{~m}, 1 \mathrm{H}), 6.28(\mathrm{dd}, 1 \mathrm{H}, J$ $=15.3,9.6 \mathrm{~Hz}), 6.62(\mathrm{~d}, 1 \mathrm{H}, J=9.6 \mathrm{~Hz}), 6.87(\mathrm{~d}, 2 \mathrm{H}, J=8.7 \mathrm{~Hz}), 7.24(\mathrm{~d}, 2 \mathrm{H}, J=8.7$ $\mathrm{Hz}) ;{ }^{13} \mathrm{C}-\mathrm{NMR}\left(100 \mathrm{MHz}, \mathrm{CDCl}_{3}\right) \delta 12.0,18.0,24.5,29.9,31.0,39.6,44.0,51.9,55.2$, 71.6, 72.9, 74.2, 75.2, 104.7, 113.8, 129.4, 131.5, 132.9, 135.8, 159.3; HRMS (FAB, $m$ $\mathrm{NBA}+\mathrm{NaI})[\mathrm{M}+\mathrm{Na}]^{+}$calcd for $\mathrm{C}_{30} \mathrm{H}_{49} \mathrm{IO}_{4} \mathrm{SiNa} 651.2342$, found 651.2367.

$(3 S, 4 S, 6 S, 8 R, 10 S)-((S, 3 E, 5 Z)-6-B r o m o-1-\{(1 R, 2 R)-2-[(4-$ methoxybenzyloxy)methyl]cyclopentyl\}-7-(triisopropylsilyloxy)hepta-3,5-dienyl) 3(tert-butyldimethylsilyloxy)-11-hydroxy-4,6,8,10-tetramethylundecanoate (62a)
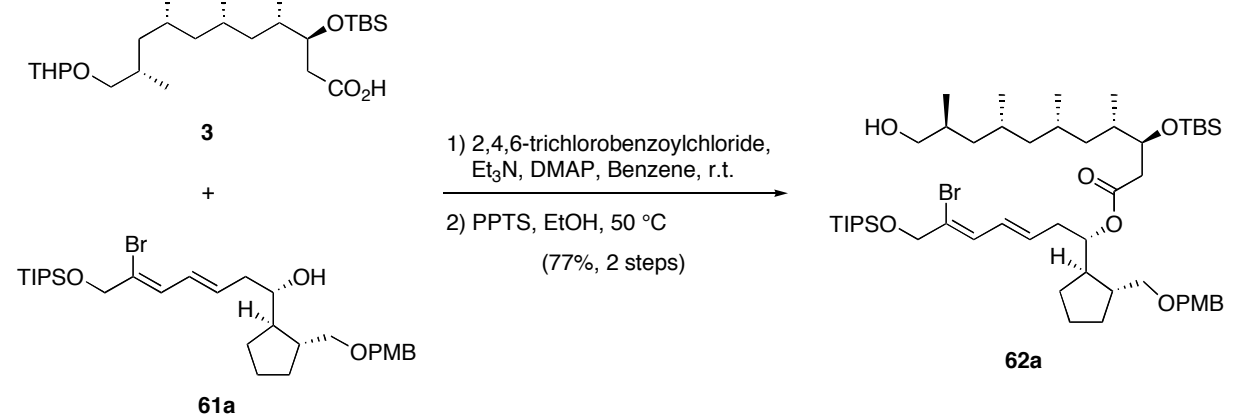

A solution of $3(13.4 \mathrm{mg}, 28.4 \mu \mathrm{mol})$ in benzene $(0.4 \mathrm{ml})$ at room temperature was 
treated with $\mathrm{Et}_{3} \mathrm{~N}(8 \mu \mathrm{L}, 56.8 \mu \mathrm{mol})$ and 2,4,6-trichlorobenzoyl chloride $(5 \mu \mathrm{L}, 31.2$ $\mu \mathrm{mol})$ under an argon atmosphere. After stirring for $1 \mathrm{~h}$, a solution of 61a $(22.1 \mathrm{mg}$, $38.1 \mu \mathrm{mol})$ in benzene $(1 \mathrm{ml})$ followed by DMAP $(4.50 \mathrm{mg}, 36.9 \mu \mathrm{mol})$ were added. After stirring for $2 \mathrm{~h}$ at room temperature, the resulting mixture was treated with aqueous $\mathrm{NaHCO}_{3}$ solution and extracted with EtOAc. The combined organic phases were dried over anhydrous $\mathrm{Na}_{2} \mathrm{SO}_{4}$ and concentrated in vacuo. The crude product was purified by column chromatography $(60: 1$ hexanes/EtOAc) to give the corresponding ester.

To a solution of the ester in EtOH $(0.9 \mathrm{ml})$ at room temperature was added PPTS (3.90 $\mathrm{mg}, 15.5 \mu \mathrm{mol})$. After stirring for $8 \mathrm{~h}$ at $50^{\circ} \mathrm{C}$, the reaction mixture was diluted with water and extracted with EtOAc. The combined organic phases were dried over anhydrous $\mathrm{Na}_{2} \mathrm{SO}_{4}$ and concentrated in vacuo. The crude product was purified by column chromatography (10:1 hexanes/EtOAc) to give 62a $(20.9 \mathrm{mg}, 77 \%$ for 2 steps). ${ }^{1} \mathrm{H}-\mathrm{NMR}\left(300 \mathrm{MHz}, \mathrm{CDCl}_{3}\right) \delta 0.02$ (s, 3H), 0.05 (s, 3H), 0.80-1.39 (m, 49H), 1.48-1.76 (m, 9H), $1.84(\mathrm{~m}, 1 \mathrm{H}), 2.06(\mathrm{~m}, 1 \mathrm{H}), 2.30-2.48(\mathrm{~m}, 4 \mathrm{H}), 3.21(\mathrm{t}, 1 \mathrm{H}, J=8.7$ $\mathrm{Hz}), 3.35-3.47(\mathrm{~m}, 3 \mathrm{H}), 3.79(\mathrm{~s}, 3 \mathrm{H}), 4.06(\mathrm{dt}, 1 \mathrm{H}, J=6.3,2.5 \mathrm{~Hz}), 4.36(\mathrm{~s}, 2 \mathrm{H}), 4.39$ $(\mathrm{d}, 1 \mathrm{H}, J=11.8 \mathrm{~Hz}), 4.46(\mathrm{~d}, 1 \mathrm{H}, J=11.8 \mathrm{~Hz}), 4.90(\mathrm{~m}, 1 \mathrm{H}), 5.78(\mathrm{~m}, 1 \mathrm{H}), 6.36(\mathrm{dd}$, $1 \mathrm{H}, J=15.2,10.1 \mathrm{~Hz}), 6.62(\mathrm{~d}, 1 \mathrm{H}, J=10.1 \mathrm{~Hz}), 6.68(\mathrm{~d}, 2 \mathrm{H}, J=8.7 \mathrm{~Hz}), 7.24(\mathrm{~d}, 2 \mathrm{H}$, $J=8.7 \mathrm{~Hz})$
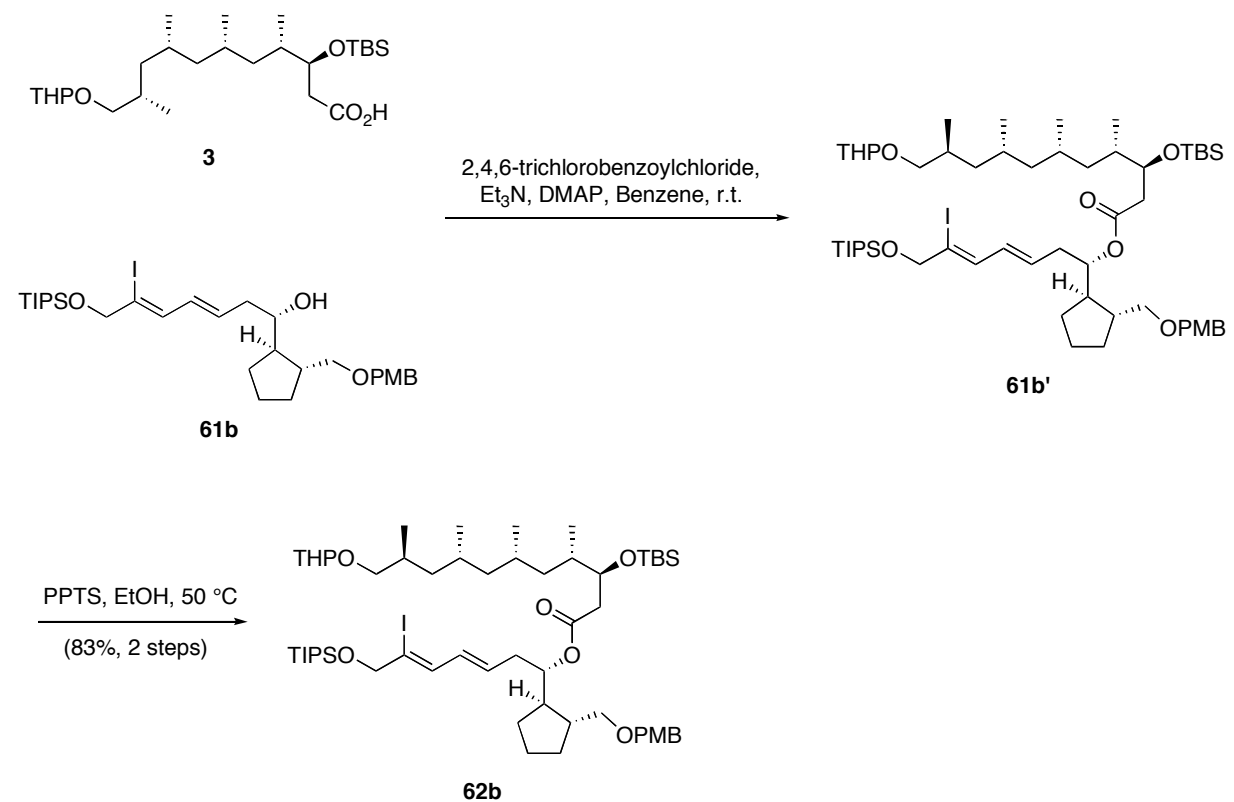

$(3 S, 4 S, 6 S, 8 R, 10 S)-((S, 3 E, 5 Z)-6-I o d o-1-\{(1 R, 2 R)-2-[(4-$

methoxybenzyloxy)methyl]cyclopentyl\}-7-(triisopropylsilyloxy)hepta-3,5-dienyl) 3(tert-butyldimethylsilyloxy)-4,6,8,10-tetramethyl-11-(tetrahydro-2H-pyran-2yloxy)undecanoate (61b') 


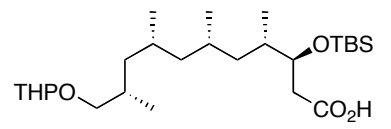

3

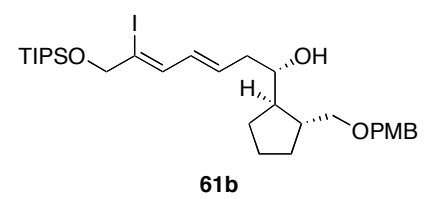

2,4,6-trichlorobenzoylchloride, $\mathrm{Et}_{3} \mathrm{~N}$, DMAP, Benzene, r.t.

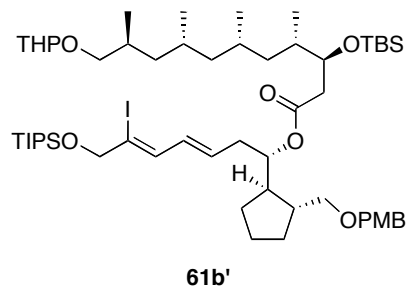

A solution of $3(44.3 \mathrm{mg}, 0.094 \mathrm{mmol})$ in benzene $(0.7 \mathrm{ml})$ at room temperature was treated with $\mathrm{Et}_{3} \mathrm{~N}(26 \mu \mathrm{L}, 0.188 \mathrm{mmol})$ and 2,4,6-trichlorobenzoyl chloride $(16 \mu \mathrm{L}$, $0.103 \mathrm{mmol}$ ) under an argon atmosphere. After stirring for $1 \mathrm{~h}$, a solution of $\mathbf{6 1 b}$ (74.2 $\mathrm{ml}, 0.118 \mathrm{mmol})$ in benzene $(1.2 \mathrm{ml})$ was added dropwise and DMAP was then added. After stirring for $2 \mathrm{~h}$ at room temperature, the resulting mixture was treated with aqueous $\mathrm{NaHCO}_{3}$ solution and extracted with EtOAc. The combined organic phases were dried over anhydrous $\mathrm{Na}_{2} \mathrm{SO}_{4}$ and concentrated in vacuo. The crude product was purified by column chromatography $(60: 1$ hexanes/EtOAc) to give a mixture of $\mathbf{6 1 b}$ ' and $61 \mathrm{~b}$.

61b': $[\alpha]^{27}{ }_{\mathrm{D}}-8.4^{\circ}\left(c \mathrm{0.17}, \mathrm{CHCl}_{3}\right)$; IR (KBr) 2945, 2862, 2357, 1734, 1460, 1373, 1250, 1116, 1032, 827, $679 \mathrm{~cm}^{-1}$; ${ }^{1} \mathrm{H}-\mathrm{NMR}\left(300 \mathrm{MHz}, \mathrm{CDCl}_{3}\right) \delta 0.03(\mathrm{~s}, 3 \mathrm{H}), 0.06(\mathrm{~s}, 3 \mathrm{H})$, 0.79-0.90 (m, 23H), 0.95-1.41 (m, 26H), 1.49-1.87 (m, 16H), $2.07(\mathrm{~m}, 1 \mathrm{H}), 2.32-2.52$ $(\mathrm{m}, 4 \mathrm{H}), 3.09-3.27(\mathrm{~m}, 2 \mathrm{H}), 3.36-3.58(\mathrm{~m}, 3 \mathrm{H}), 3.80(\mathrm{~s}, 3 \mathrm{H}), 3.85(\mathrm{~m}, 1 \mathrm{H}), 4.07(\mathrm{~m}$, $1 \mathrm{H}), 4.37(\mathrm{~s}, 2 \mathrm{H}), 4.42(\mathrm{~d}, 1 \mathrm{H}, J=11.7 \mathrm{~Hz}), 4.47(\mathrm{~d}, 1 \mathrm{H}, J=11.7 \mathrm{~Hz}), 4.57(\mathrm{~m}, 1 \mathrm{H})$, $4.92(\mathrm{~m}, 1 \mathrm{H}), 5.83(\mathrm{~m}, 1 \mathrm{H}), 6.24(\mathrm{dd}, 1 \mathrm{H}, J=15.0,9.9 \mathrm{~Hz}), 6.56(\mathrm{~d}, 1 \mathrm{H}, J=9.9 \mathrm{~Hz})$, $6.87(\mathrm{~d}, 2 \mathrm{H}, J=8.8 \mathrm{~Hz}), 7.25(\mathrm{~d}, 2 \mathrm{H}, J=8.8 \mathrm{~Hz}) ;{ }^{13} \mathrm{C}-\mathrm{NMR}\left(100 \mathrm{MHz}, \mathrm{CDCl}_{3}\right) \delta-4.6$, $-4.1,12.1,12.2,14.5,16.6,16.7,18.1,18.2,19.6,19.7,20.6,20.8,25.3,25.7,26.0$, $27.1,27.2,27.3,29.8,30.5,31.0,31.0,35.5,36.4,40.1,41.6,44.7,46.0,55.3,62.1$, 62.2, 71.6, 71.7, 72.8, 74.0, 74.1, 74.2, 98.7, 99.1, 106.1, 109.3, 113.8, 129.1, 130.8, 131.1, 133.4, 134.2, 159.1, 171.8; HRMS (FAB, $m-\mathrm{NBA}+\mathrm{NaI})[\mathrm{M}+\mathrm{Na}]^{+}$calcd for $\mathrm{C}_{56} \mathrm{H}_{99} \mathrm{IO}_{8} \mathrm{Si}_{2} \mathrm{Na}$ 1105.5821, found 1105.5821.

$(3 S, 4 S, 6 S, 8 R, 10 S)-((S, 3 E, 5 Z)-6-I o d o-1-\{(1 R, 2 R)-2-[(4-$ methoxybenzyloxy)methyl]cyclopentyl $\}-7-($ triisopropylsilyloxy)hepta-3,5-dienyl) 3(tert-butyldimethylsilyloxy)-11-hydroxy-4,6,8,10-tetramethylundecanoate $(62 \mathrm{~b})$

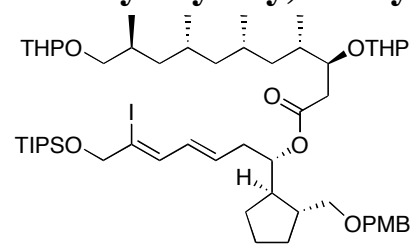

61b'
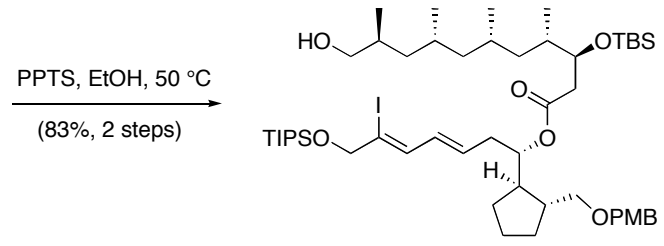

$62 b$

To a solution of the mixture of $\mathbf{6 1 b}$ ' and $\mathbf{6 1 b}$ in EtOH $(3.5 \mathrm{ml})$ at room temperature was added PPTS (13.2 mg, $0.052 \mathrm{mmol}$ ). After stirring for $12.5 \mathrm{~h}$ at $50^{\circ} \mathrm{C}$, the reaction mixture was diluted with water and extracted with EtOAc. The combined organic phases were dried over anhydrous $\mathrm{Na}_{2} \mathrm{SO}_{4}$ and concentrated in vacuo. The crude product was purified by column chromatography (40:1 hexanes/EtOAc) to give $62 \mathbf{b}$ (77.4 $\mathrm{mg}$, $83 \%$ for 2 steps). $[\alpha]^{24}-15.5^{\circ}\left(c 0.17, \mathrm{CHCl}_{3}\right)$; IR (KBr) 3464, 2951, 2862, 1732, 1514 , 
1462, 1375, 1250, 1113, 833, 781, $679 \mathrm{~cm}^{-1} ;{ }^{1} \mathrm{H}-\mathrm{NMR}\left(300 \mathrm{MHz}, \mathrm{CDCl}_{3}\right) \delta 0.03$ (s, $3 \mathrm{H}), 0.06(\mathrm{~s}, 3 \mathrm{H}), 0.79-1.00(\mathrm{~m}, 24 \mathrm{H}), 1.02-1.40(\mathrm{~m}, 25 \mathrm{H}), 1.49-1.78(\mathrm{~m}, 9 \mathrm{H}), 1.87(\mathrm{~m}$, $1 \mathrm{H}), 2.07(\mathrm{~m}, 1 \mathrm{H}), 2.31-2.52(\mathrm{~m}, 4 \mathrm{H}), 3.22(\mathrm{t}, 1 \mathrm{H}, J=8.4 \mathrm{~Hz}), 3.41(\mathrm{dd}, 1 \mathrm{H}, J=8.4,6.4$ $\mathrm{Hz}), 3.35-3.47(\mathrm{~m}, 2 \mathrm{H}), 3.80(\mathrm{~s}, 3 \mathrm{H}), 4.07(\mathrm{dt}, 1 \mathrm{H}, J=6.4,2.4 \mathrm{~Hz}), 4.37(\mathrm{~s}, 2 \mathrm{H}), 4.40$ $(\mathrm{d}, 1 \mathrm{H}, J=11.8 \mathrm{~Hz}), 4.42(\mathrm{~d}, 1 \mathrm{H}, J=11.8 \mathrm{~Hz}), 4.91(\mathrm{~m}, 1 \mathrm{H}), 5.83(\mathrm{dt}, 1 \mathrm{H}, J=15.0,7.4$ $\mathrm{Hz}), 6.24(\mathrm{dd}, 1 \mathrm{H}, J=15.0,9.8 \mathrm{~Hz}), 6.56(\mathrm{~d}, 1 \mathrm{H}, J=9.8 \mathrm{~Hz}), 6.87(\mathrm{~d}, 2 \mathrm{H}, J=8.7 \mathrm{~Hz})$, $7.25(\mathrm{~d}, 2 \mathrm{H}, J=8.7 \mathrm{~Hz}) ;{ }^{13} \mathrm{C}-\mathrm{NMR}\left(100 \mathrm{MHz}, \mathrm{CDCl}_{3}\right) \delta-4.7,-4.3,12.0,14.4,16.0$, $18.0,18.1,20.6,20.7,25.2,25.9,27.1,27.3,29.8,30.4,33.3,35.4,36.4,39.6,40.0$, 41.3, 41.5, 44.6, 45.8, 55.2, 69.3, 71.6, 71.7, 72.7, 74.1, 76.4, 106.0, 113.7, 129.0, 130.7, 131.0, 133.3, 134.1, 159.0, 171.7; HRMS (FAB, $m-\mathrm{NBA}+\mathrm{NaI})[\mathrm{M}+\mathrm{Na}]^{+}$calcd for $\mathrm{C}_{51} \mathrm{H}_{91} \mathrm{IO}_{7} \mathrm{Si}_{2} \mathrm{Na}$ 1021.5246, found 1021.5275.

$(3 S, 4 S, 6 S, 8 R, 10 S)-((S, 3 E, 5 Z)-6-B r o m o-1-\{(1 R, 2 R)-2-[(4-$ methoxybenzyloxy)methyl]cyclopentyl\}-7-(triisopropylsilyloxy)hepta-3,5-dienyl) 3(tert-butyldimethylsilyloxy)-4,6,8,10-tetramethyl-11-oxoundecanoate (58a)
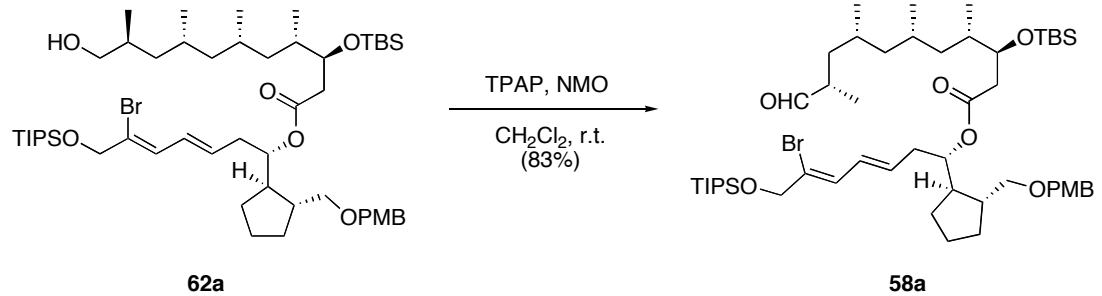

To a mixture of dried MS4A (10.0 mg) and 62a (19.9 mg, $20.9 \mu \mathrm{mol})$ in $\mathrm{CH}_{2} \mathrm{Cl}_{2}(1.1$ $\mathrm{ml})$ were added NMO (4.90 mg, $41.8 \mu \mathrm{mol})$ and TPAP $(0.40 \mathrm{mg}, 1.0 \mu \mathrm{mol})$ at room temperature under a argon atmosphere. After stirring for 15 minute at room temperature, additional NMO $(4.90 \mathrm{mg}, 41.8 \mu \mathrm{mol})$ and TPAP $(0.40 \mathrm{mg}, 1.0 \mu \mathrm{mol})$ were added. After 30 minute, the reaction mixture was treated with aqueous $\mathrm{Na}_{2} \mathrm{~S}_{2} \mathrm{O}_{3}$ solution, filtered through a pad of Celite, and extracted with EtOAc. The combined organic layers were dried over anhydrous $\mathrm{Na}_{2} \mathrm{SO}_{4}$ and removed in vacuo. The crude product was purified by column chromatography (30:1 hexanes/EtOAc) to give aldehyde 58a (16.4 $\mathrm{mg}, 83 \%) .{ }^{1} \mathrm{H}-\mathrm{NMR}\left(300 \mathrm{MHz}, \mathrm{CDCl}_{3}\right) \delta 0.02$ (s, 3H), $0.04(\mathrm{~s}, 3 \mathrm{H}), 0.79-0.99$ (m, $20 \mathrm{H}), 1.04-1.41(\mathrm{~m}, 30 \mathrm{H}), 1.46-1.76(\mathrm{~m}, 7 \mathrm{H}), 1.84(\mathrm{~m}, 1 \mathrm{H}), 2.06(\mathrm{~m}, 1 \mathrm{H}), 2.30-2.50(\mathrm{~m}$, $5 \mathrm{H}), 3.21(\mathrm{t}, 1 \mathrm{H}, J=8.7 \mathrm{~Hz}), 3.39(\mathrm{dd}, 1 \mathrm{H}, J=8.7,5.1 \mathrm{~Hz}), 3.79(\mathrm{~s}, 3 \mathrm{H}), 4.06(\mathrm{dt}, 1 \mathrm{H}, J$ $=6.2,2.4 \mathrm{~Hz}), 4.35(\mathrm{~s}, 2 \mathrm{H}), 4.39(\mathrm{~d}, 1 \mathrm{H}, J=11.7 \mathrm{~Hz}), 4.45(\mathrm{~d}, 1 \mathrm{H}, J=11.7 \mathrm{~Hz}), 4.90$ $(\mathrm{m}, 1 \mathrm{H}), 5.78(\mathrm{~m}, 1 \mathrm{H}), 6.36(\mathrm{dd}, 1 \mathrm{H}, J=15.1,10.2 \mathrm{~Hz}), 6.62(\mathrm{~d}, 1 \mathrm{H}, J=10.2 \mathrm{~Hz}), 6.68$ $(\mathrm{d}, 1 \mathrm{H}, J=8.6 \mathrm{~Hz}), 7.24(\mathrm{~d}, 1 \mathrm{H}, J=8.6 \mathrm{~Hz}), 9.60(\mathrm{~d}, 1 \mathrm{H}, J=1.6 \mathrm{~Hz})$; HRMS (FAB, $m-$ $\mathrm{NBA}+\mathrm{NaI})[\mathrm{M}+\mathrm{Na}]^{+}$calcd for $\mathrm{C}_{51} \mathrm{H}_{89} \mathrm{BrO}_{7} \mathrm{Si}_{2} \mathrm{Na}$ 971.5228, found 971.5195.

$(3 S, 4 S, 6 S, 8 R, 10 S)-((S, 3 E, 5 Z)-6-I o d o-1-\{(1 R, 2 R)-2-[(4-$ methoxybenzyloxy)methyl]cyclopentyl $\}-7-($ triisopropylsilyloxy)hepta-3,5-dienyl) 3(tert-butyldimethylsilyloxy)-4,6,8,10-tetramethyl-11-oxoundecanoate (58b)

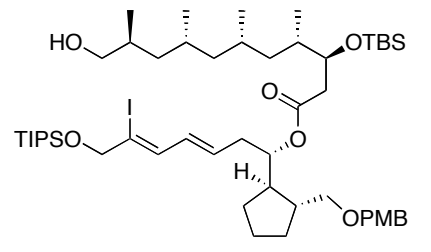

$62 b$

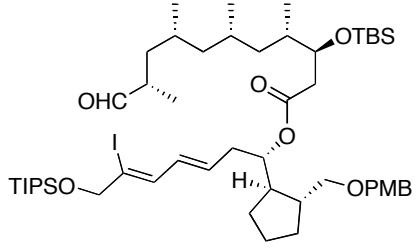

$58 b$ 
To a mixture of dried MS4 $\AA$ (35.0 mg) and $\mathbf{6 2 b}(66.5 \mathrm{mg}, 0.070 \mathrm{mmol})$ in $\mathrm{CH}_{2} \mathrm{Cl}_{2}(3.5$ $\mathrm{ml})$ were added NMO (16.5 mg, $0.140 \mathrm{mmol})$ and TPAP $(1.20 \mathrm{mg}, 3.5 \mu \mathrm{mol})$ at room temperature under a nitrogene atmosphere. After stirring for $15 \mathrm{~min}$ at room temperature, the reaction mixture was treated with aqueous $\mathrm{Na}_{2} \mathrm{~S}_{2} \mathrm{O}_{3}$ solution, filtered through a pad of Celite, and extracted with EtOAc. The combined organic layers were dried over anhydrous $\mathrm{Na}_{2} \mathrm{SO}_{4}$ and removed in vacuo. The crude product was purified by column chromatography ( $30: 1$ hexanes/EtOAc) to give aldehyde $\mathbf{5 8 b}(54.6 \mathrm{mg}, 82 \%)$. $[\alpha]^{24}+1.1^{\circ}\left(c 0.48, \mathrm{CHCl}_{3}\right)$ IR $(\mathrm{KBr}) 2935,2862,1732,1514,1462,1375,1250,1113$, 833, $679 \mathrm{~cm}^{-1}$; ${ }^{1} \mathrm{H}-\mathrm{NMR}\left(300 \mathrm{MHz}, \mathrm{CDCl}_{3}\right) \delta 0.02$ (s, 3H), 0.05 (s, 3H), 0.79-1.00 (m, $20 \mathrm{H}), 1.12-1.90(\mathrm{~m}, 38 \mathrm{H}), 2.06(\mathrm{~m}, 1 \mathrm{H}), 2.30-2.51(\mathrm{~m}, 5 \mathrm{H}), 3.21(\mathrm{t}, 1 \mathrm{H}, J=8.7 \mathrm{~Hz})$, $3.39(\mathrm{dd}, 1 \mathrm{H}, J=8.7,5.4 \mathrm{~Hz}), 3.79(\mathrm{~s}, 3 \mathrm{H}), 4.06(\mathrm{~m}, 1 \mathrm{H}), 4.36(\mathrm{~s}, 2 \mathrm{H}), 4.39(\mathrm{~d}, 1 \mathrm{H}, J=$ $11.7 \mathrm{~Hz}), 4.46(\mathrm{~d}, 1 \mathrm{H}, J=11.7 \mathrm{~Hz}), 4.91(\mathrm{~m}, 1 \mathrm{H}), 5.83(\mathrm{~m}, 1 \mathrm{H}), 6.23(\mathrm{dd}, 1 \mathrm{H}, J=15.0$, $9.7 \mathrm{~Hz}), 6.55(\mathrm{~d}, 1 \mathrm{H}, J=9.7 \mathrm{~Hz}), 6.86(\mathrm{~d}, 2 \mathrm{H}, J=8.2 \mathrm{~Hz}), 7.24(\mathrm{~d}, 2 \mathrm{H}, J=8.2 \mathrm{~Hz})$, $9.60(\mathrm{~d}, 1 \mathrm{H}, J=1.7 \mathrm{~Hz}) ;{ }^{13} \mathrm{C}-\mathrm{NMR}\left(100 \mathrm{MHz}, \mathrm{CDCl}_{3}\right) \delta-4.7,-4.2,12.0,12.0,13.0$, 14.4, 18.0, 18.1, 20.4, 20.5, 25.2, 25.9, 27.3, 29.8, 30.4, 35.4, 36.3, 36.4, 39.9, 41.0, 41.5, 44.3, 44.6, 45.4, 55.2, 71.5, 71.6, 72.7, 74.1, 76.4, 106.0, 113.7, 129.0, 130.7, 131.0, 133.3, 134.1, 159.0, 171.6, 205.2; HRMS (FAB, $m-\mathrm{NBA}+\mathrm{NaI})[\mathrm{M}+\mathrm{Na}]^{+}$calcd for $\mathrm{C}_{51} \mathrm{H}_{89} \mathrm{IO}_{7} \mathrm{Si}_{2} \mathrm{Na} 1019.5089$, found 1019.5070. 
$(4 S, 5 S, 7 S, 9 R, 11 S, 13 Z, 15 E, 18 S)-4$-(tert-Butyldimethylsilyloxy)-12-hydroxy-18$\{(1 R, 2 R)-2-[(4-m e t h o x y b e n z y l o x y)$ methyl]cyclopentyl $\}-5,7,9,11-t e t r a m e t h y l-13-$ [(triisopropylsilyloxy)methyl]oxacyclooctadeca-13,15-dien-2-one (57)

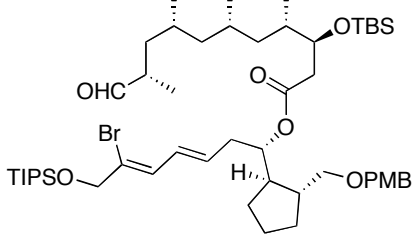

$58 \mathrm{a}$
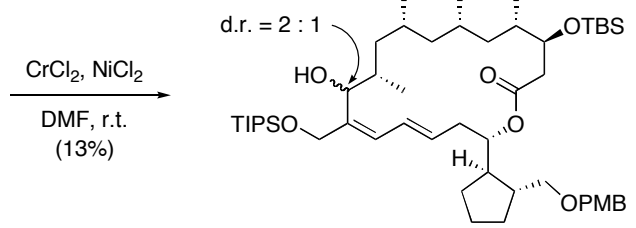

57

To a solution of $\mathrm{CrCl}_{2}(25.1 \mathrm{mg}, 0.204 \mathrm{mmol})$ and $\mathrm{NiCl}_{2}(0.30 \mathrm{mg}, 2.30 \mu \mathrm{mol})$ in $\mathrm{DMF}$ $(0.5 \mathrm{ml})$ at room teperature under argon was added a solution of $\mathbf{5 8 a}(15.5 \mathrm{mg}, 16.3$ $\mu \mathrm{mol})$ in DMF $(1.1 \mathrm{ml})$. After stirring for $12 \mathrm{~h}$ at room teperature, the reaction mixture was filtered through a pad of Celite and extracted with EtOAc. The combined organic phases were dried over anhydrous $\mathrm{Na}_{2} \mathrm{SO}_{4}$ and concentrated in vacuo. The crude product was purified by column chromatography (30:1 hexanes/EtOAc) to give the unreacted starting material 58a (10.0 mg, 68\%) and $\mathbf{5 7}(1.10 \mathrm{mg}, 13 \%)$.

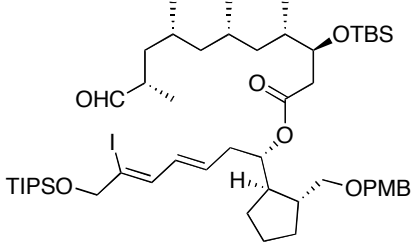

$58 b$

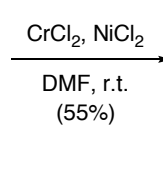

$F$, r.t.

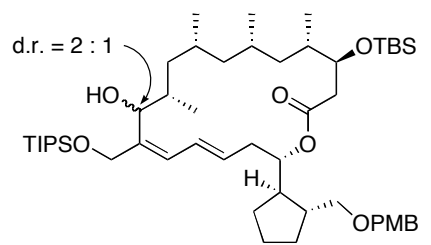

57

To a solution of $\mathrm{CrCl}_{2}(61.6 \mathrm{mg}, 0.501 \mathrm{mmol})$ and $\mathrm{NiCl}_{2}(0.60 \mathrm{mg}, 4.70 \mu \mathrm{mol})$ in $\mathrm{DMF}$ $(1.9 \mathrm{ml})$ at room teperature under argon was added a solution of $\mathbf{5 8 b}(44.3 \mathrm{mg}, 0.047$ $\mathrm{mmol})$ in DMF (2.8 ml). After stirring for $14 \mathrm{~h}$ at room teperature, the reaction mixture was filtered through a pad of Celite and extracted with EtOAc. The combined organic phases were dried over anhydrous $\mathrm{Na}_{2} \mathrm{SO}_{4}$ and concentrated in vacuo. The crude product was purified by column chromatography (40:1 hexanes/EtOAc) to give $\mathbf{5 7}(22.2 \mathrm{mg}$, $55 \%)$.

Major isomer: $[\alpha]_{\mathrm{D}}^{25}=-19.2^{\circ}\left(c=0.20, \mathrm{CHCl}_{3}\right)$; IR $(\mathrm{KBr}) 2927,2860,1734,1514$, 1462, 1375, 1250, 1173, 1082, $833679 \mathrm{~cm}^{-1} ;{ }^{1} \mathrm{H}-\mathrm{NMR}\left(300 \mathrm{MHz}, \mathrm{CDCl}_{3}\right) \delta 0.08(\mathrm{~s}$, $3 \mathrm{H}), 0.11(\mathrm{~s}, 3 \mathrm{H}), 0.77-1.18(\mathrm{~m}, 46 \mathrm{H}), 1.50-2.46(\mathrm{~m}, 16 \mathrm{H}), 3.15(\mathrm{t}, 1 \mathrm{H}, J=9.0 \mathrm{~Hz}), 3.35$ $(\mathrm{dd}, 1 \mathrm{H}, J=9.0,5.0 \mathrm{~Hz}), 3.79(\mathrm{~s}, 3 \mathrm{H}), 4.04(\mathrm{~m}, 1 \mathrm{H}), 4.14(\mathrm{~d}, 1 \mathrm{H}, J=10.0 \mathrm{~Hz}), 4.17(\mathrm{~d}$, $1 \mathrm{H}, J=12.1 \mathrm{~Hz},), 4.40(\mathrm{~s}, 2 \mathrm{H}), 4.64(\mathrm{~d}, 1 \mathrm{H}, J=12.1 \mathrm{~Hz}), 4.98(\mathrm{~m}, 1 \mathrm{H}), 5.79(\mathrm{~m}, 1 \mathrm{H})$, $6.13(\mathrm{~d}, 1 \mathrm{H}, J=10.9 \mathrm{~Hz}), 6.28(\mathrm{dd}, 1 \mathrm{H}, J=14.4,10.9 \mathrm{~Hz}), 6.85(\mathrm{~d}, 2 \mathrm{H}, J=8.7 \mathrm{~Hz})$, $7.23(\mathrm{~d}, 2 \mathrm{H}, J=8.7 \mathrm{~Hz}) ;{ }^{13} \mathrm{C}-\mathrm{NMR}\left(100 \mathrm{MHz}, \mathrm{CDCl}_{3}\right) \delta-5.4,-3.6,11.8,11.9,14.1$, $15.4,17.7,18.0,18.6,19.8,24.9,25.9,26.1,30.0,33.5,34.6,35.6,35.7,36.5,38.1$, 42.7, 43.4, 43.6, 48.3, 55.2, 67.2, 72.4, 73.7, 74.1, 75.7, 76.0, 113.8, 128.4, 128.9, 129.3, 130.0, 130.8; HRMS (FAB, $m-\mathrm{NBA}+\mathrm{NaI}$ ) $[\mathrm{M}+\mathrm{Na}]^{+}$calcd for $\mathrm{C}_{51} \mathrm{H}_{91} \mathrm{O}_{7} \mathrm{Si}_{2} \mathrm{Na}$ 893.6123, found 893.6139.

$(S, 2 Z, 4 E)-2-B r o m o-7-(t e r t-b u t y l d i m e t h y l s i l y l o x y)-7-\{(1 R, 2 R)-2-[(4-$ methoxybenzyloxy)methyl]cyclopentyl\}hepta-2,4-dienal (30) 


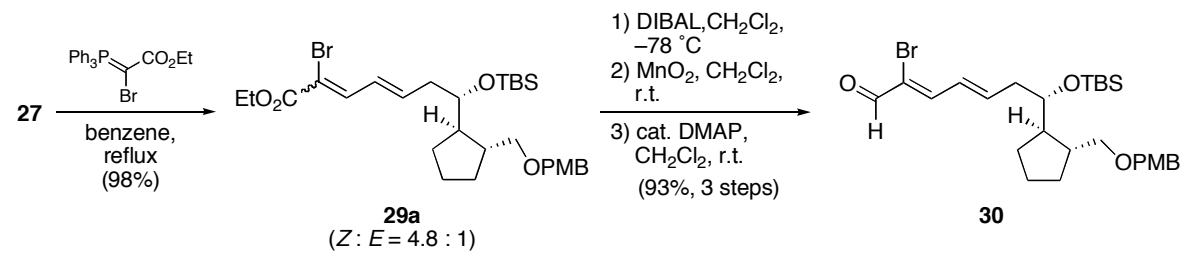

A solution of $27(2.15 \mathrm{~g}, 4.97 \mathrm{mmol})$ in benzene $(49.7 \mathrm{ml})$ was treated with (ethoxycarbonylbromomethylidene)triphenylphosphorane $(6.35 \mathrm{~g}, 14.9 \mathrm{mmol}$ ) and refluxed at $80{ }^{\circ} \mathrm{C}$ for $12 \mathrm{~h}$. The reaction mixture was concentrated in vacuo and roughly purified by column chromatography (30:1 hexanes/EtOAc) to give the $E, Z$ mixture of 29a $(2.83 \mathrm{~g}, 98 \%)$.

To a solution of the $E, Z$ mixture of $29 \mathbf{a}(2.83 \mathrm{mg}, 4.88 \mathrm{mmol})$ in $\mathrm{CH}_{2} \mathrm{Cl}_{2}(49 \mathrm{ml})$ was added dropwise DIBAL $(0.95 \mathrm{M}$ in hexane, $12.8 \mathrm{ml}, 12.2 \mathrm{mmol})$ at $0^{\circ} \mathrm{C}$. After stirring for $0.5 \mathrm{~h}$, the reaction was quenched with $\mathrm{MeOH}$, diluted with $\mathrm{CH}_{2} \mathrm{Cl}_{2}$, and treated with Celite $(30 \mathrm{~g})$ and $\mathrm{Na}_{2} \mathrm{SO}_{4} \cdot 10 \mathrm{H}_{2} \mathrm{O}(30 \mathrm{~g})$. The resulting mixture was stirred for $2 \mathrm{~h}$ at room temperature, filtered through a pad of Celite, and concentrated in vacuo to give the corresponding alcohol.

The crude alcohol was dissolved in $\mathrm{CH}_{2} \mathrm{Cl}_{2}(49 \mathrm{ml})$ and treated with manganese dioxide $(12.7 \mathrm{~g}, 146 \mathrm{mmol})$. After stirring for $12 \mathrm{~h}$ at room temperature, the resulting mixture was filtered through a pad of Celite and concentrated in vacuo to afford a $E, Z$ mixture of the corresponding aldehyde.

To a solution of the crude aldehyde in $\mathrm{CH}_{2} \mathrm{Cl}_{2}$ (49 ml) was added DMAP (366 mg, 2.99 $\mathrm{mmol}$ ). The mixture was stirred for $24 \mathrm{~h}$ at room temperature, diluted with water, and extracted with $\mathrm{CH}_{2} \mathrm{Cl}_{2}$. The combined organic extracts were dried over anhydrous $\mathrm{Na}_{2} \mathrm{SO}_{4}$ and concentrated in vacuo. Flash chromatography (30:1 hexanes/EtOAc) afforded 30 (2.43 g, 93\% for 3 steps) as a colorless oil. ${ }^{1} \mathrm{H}-\mathrm{NMR}\left(270 \mathrm{MHz}, \mathrm{CDCl}_{3}\right) \delta$ $0.04(\mathrm{~s}, 3 \mathrm{H}), 0.06(\mathrm{~s}, 3 \mathrm{H}), 0.88(\mathrm{~s}, 9 \mathrm{H}), 1.30(\mathrm{~m}, 1 \mathrm{H}), 1.45-1.75(\mathrm{~m}, 6 \mathrm{H}), 2.09(\mathrm{~m}, 1 \mathrm{H})$, $2.45(\mathrm{~m}, 2 \mathrm{H}), 3.22(\mathrm{t}, 1 \mathrm{H}, J=8.6 \mathrm{~Hz}), 3.40(\mathrm{dd}, 1 \mathrm{H}, J=8.6,5.6 \mathrm{~Hz}), 3.75(\mathrm{~m}, 1 \mathrm{H}), 3.79$ $(\mathrm{s}, 3 \mathrm{H}), 4.40(\mathrm{~d}, 1 \mathrm{H}, J=11.9 \mathrm{~Hz}), 4.44(\mathrm{~d}, 1 \mathrm{H}, J=11.9 \mathrm{~Hz}), 6.51(\mathrm{~m}, 1 \mathrm{H}), 6.69(\mathrm{dd}, 1 \mathrm{H}$, $J=15.2,10.6 \mathrm{~Hz}), 6.86(\mathrm{~d}, 2 \mathrm{H}, J=8.2 \mathrm{~Hz}), 7.24(\mathrm{~d}, 2 \mathrm{H}, J=8.2 \mathrm{~Hz}), 7.36(\mathrm{~d}, 1 \mathrm{H}, J=$ $10.5 \mathrm{~Hz}), 9.21(\mathrm{~s}, 1 \mathrm{H}) ;{ }^{13} \mathrm{C}-\mathrm{NMR}\left(67.5 \mathrm{MHz}, \mathrm{CDCl}_{3}\right) \delta-4.5,-4.2,18.0,25.2,25.9$, 29.3, 30.4, 39.4, 41.3, 47.6, 55.2, 72.8, 74.6, 74.9, 113.7, 123.9, 129.1, 130.8, 147.5, 149.1, 159.0, 186.2; HRMS (FAB, $m-\mathrm{NBA}+\mathrm{NaI}$ ) $[\mathrm{M}+\mathrm{Na}]^{+}$calcd for $\mathrm{C}_{27} \mathrm{H}_{41} \mathrm{O}_{4} \mathrm{BrSiNa}$ 559.1855 , found 559.1857 .

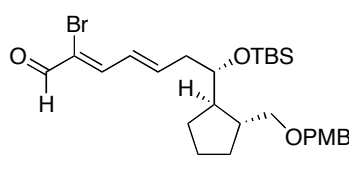

30

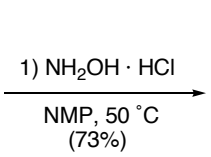

$(73 \%)$

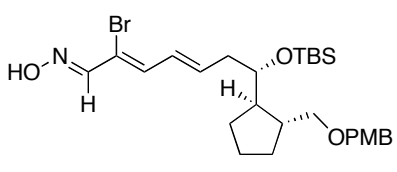

30a

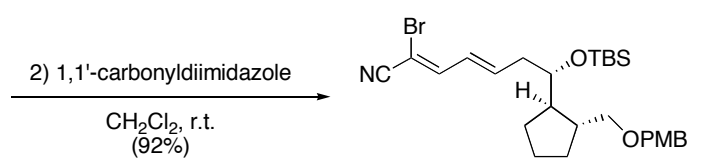

31

$(S, 1 E, 2 Z, 4 E)-2-B r o m o-7-(t e r t-b u t y l d i m e t h y l s i l y l o x y)-7-\{(1 R, 2 R)-2-[(4-$ methoxybenzyloxy)methyl]cyclopentyl\}hepta-2,4-dienal oxime (30a) 


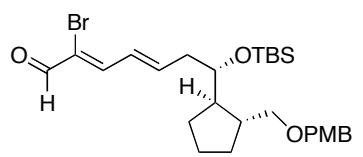

30

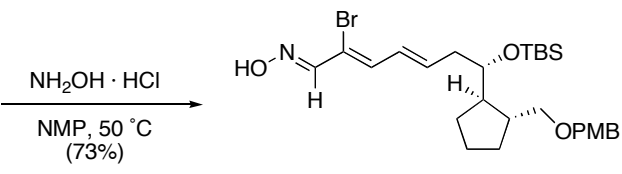

$30 a$

To a solution of $\mathbf{3 0}(19.7 \mathrm{mg}, 36.7 \mu \mathrm{mol})$ in $N$-methyl pyrolidinone (NMP, $700 \mu \mathrm{l})$ was added hydroxyamine hydrochloride $(3.00 \mathrm{mg}, 44.1 \mu \mathrm{mol})$. After stirring for $4 \mathrm{~h}$ at 50 ${ }^{\circ} \mathrm{C}$, water was added and the aqueous phase was extracted with EtOAc. The combined organic extracts were dried over anhydrous $\mathrm{Na}_{2} \mathrm{SO}_{4}$ and concentrated in vacuo. Flash chromatography (15:1 hexanes/EtOAc) afforded 30a $(14.7 \mathrm{mg}, 73 \%)$ as a colorless oil. ${ }^{1} \mathrm{H}-\mathrm{NMR}\left(270 \mathrm{MHz}, \mathrm{CDCl}_{3}\right) \delta 0.04(\mathrm{~s}, 3 \mathrm{H}), 0.05$ (s, 3H), $0.88(\mathrm{~s}, 9 \mathrm{H}), 1.29(\mathrm{~m}, 1 \mathrm{H})$, 1.46-1.71 (m, 6H), $2.09(\mathrm{~m}, 1 \mathrm{H}), 2.40(\mathrm{~m}, 2 \mathrm{H}), 3.20(\mathrm{t}, 1 \mathrm{H}, J=8.6 \mathrm{~Hz}), 3.40(\mathrm{dd}, 1 \mathrm{H}, J$ $=8.6,5.3 \mathrm{~Hz}), 3.71(\mathrm{~m}, 1 \mathrm{H}), 3.79(\mathrm{~s}, 3 \mathrm{H}), 4.39(\mathrm{~d}, 1 \mathrm{H}, J=11.9 \mathrm{~Hz}), 4.45(\mathrm{~d}, 1 \mathrm{H}, J=$ $11.9 \mathrm{~Hz}), 6.14(\mathrm{~m}, 1 \mathrm{H}), 6.55(\mathrm{dd}, 1 \mathrm{H}, J=14.8,10.2 \mathrm{~Hz}), 6.69(\mathrm{~d}, 1 \mathrm{H}, J=10.2 \mathrm{~Hz}), 6.86$ $(\mathrm{d}, 2 \mathrm{H}, J=8.2 \mathrm{~Hz}), 7.24(\mathrm{~d}, 2 \mathrm{H}, J=8.2 \mathrm{~Hz}), 7.78(\mathrm{~s}, 1 \mathrm{H}) ;{ }^{13} \mathrm{C}-\mathrm{NMR}\left(67.5 \mathrm{MHz}, \mathrm{CDCl}_{3}\right)$ $\delta$-4.6, -4.2, 18.0, 25.2, 25.9, 29.4, 30.4, 39.1, 41.2, 47.3, 55.2, 72.7, 74.6, 75.2, 113.7, 115.0, 129.2, 129.3, 130.8, 137.3, 140.0., 149.0, 159.0; HRMS (FAB, $m$-NBA+NaI) $[\mathrm{M}+\mathrm{Na}]^{+}$calcd for $\mathrm{C}_{27} \mathrm{H}_{42} \mathrm{O}_{4} \mathrm{NBrSiNa} 574.1964$, found 574.1968.

$(S, 2 Z, 4 E)-2-B r o m o-7-(t e r t-b u t y l d i m e t h y l s i l y l o x y)-7-\{(1 R, 2 R)-2-[(4-$ methoxybenzyloxy)methyl]cyclopentyl\}hepta-2,4-dienenitrile (31)

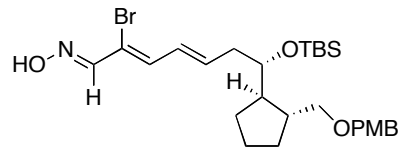

$30 \mathrm{a}$

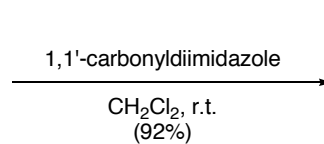

$(92 \%)$

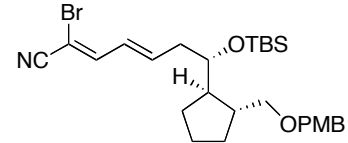

31

To a solution of $\mathbf{3 0 a}(14.7 \mathrm{mg}, 26.7 \mu \mathrm{mol})$ in $\mathrm{CH}_{2} \mathrm{Cl}_{2}(500 \mu \mathrm{l})$ was added 1,1'carbonyldiimidazole $(8.60 \mathrm{mg}, 53.4 \mu \mathrm{mol})$. After stirring for $12 \mathrm{~h}$ at room temperature, the mixture was concentrated in vacuo. Flash chromatography (30:1 hexanes/EtOAc) afforded $31(13.0 \mathrm{mg}, 92 \%)$ as a colorless oil. ${ }^{1} \mathrm{H}-\mathrm{NMR}\left(270 \mathrm{MHz}, \mathrm{CDCl}_{3}\right) \delta 0.03$ (s, $3 \mathrm{H}), 0.05(\mathrm{~s}, 3 \mathrm{H}), 0.88(\mathrm{~s}, 9 \mathrm{H}), 1.25-1.71(\mathrm{~m}, 7 \mathrm{H}), 2.07(\mathrm{~m}, 1 \mathrm{H}), 2.38(\mathrm{~m}, 2 \mathrm{H}), 3.21(\mathrm{t}$, $1 \mathrm{H}, J=8.6 \mathrm{~Hz}), 3.39(\mathrm{dd}, 1 \mathrm{H}, J=8.6,5.6 \mathrm{~Hz}), 3.72(\mathrm{~m}, 1 \mathrm{H}), 3.80(\mathrm{~s}, 3 \mathrm{H}), 4.39(\mathrm{~d}, 1 \mathrm{H}$, $J=11.9 \mathrm{~Hz}), 4.44(\mathrm{~d}, 1 \mathrm{H}, J=11.9 \mathrm{~Hz}), 6.33-6.43(\mathrm{~m}, 2 \mathrm{H}), 6.87(\mathrm{~d}, 2 \mathrm{H}, J=8.6 \mathrm{~Hz})$, $7.14(\mathrm{~m}, 1 \mathrm{H}), 7,24(\mathrm{~d}, 2 \mathrm{H}, J=8.6 \mathrm{~Hz}) ;{ }^{13} \mathrm{C}-\mathrm{NMR}\left(67.5 \mathrm{MHz}, \mathrm{CDCl}_{3}\right) \delta-4.6,-4.3,17.9$, 25.2, 25.8, 29.2, 30.3, 39.0, 41.3, 47.6, 55.2, 72.7, 74.5, 74.7, 85.7, 113.6, 116.8, 129.1, $127.9,130.6,145.7,145.8,159.0$; HRMS (FAB, $m$-NBA) $[\mathrm{M}]^{+}$calcd for $\mathrm{C}_{27} \mathrm{H}_{40} \mathrm{O}_{3} \mathrm{NBrSi}$ 533.1960, found 533.1931. 

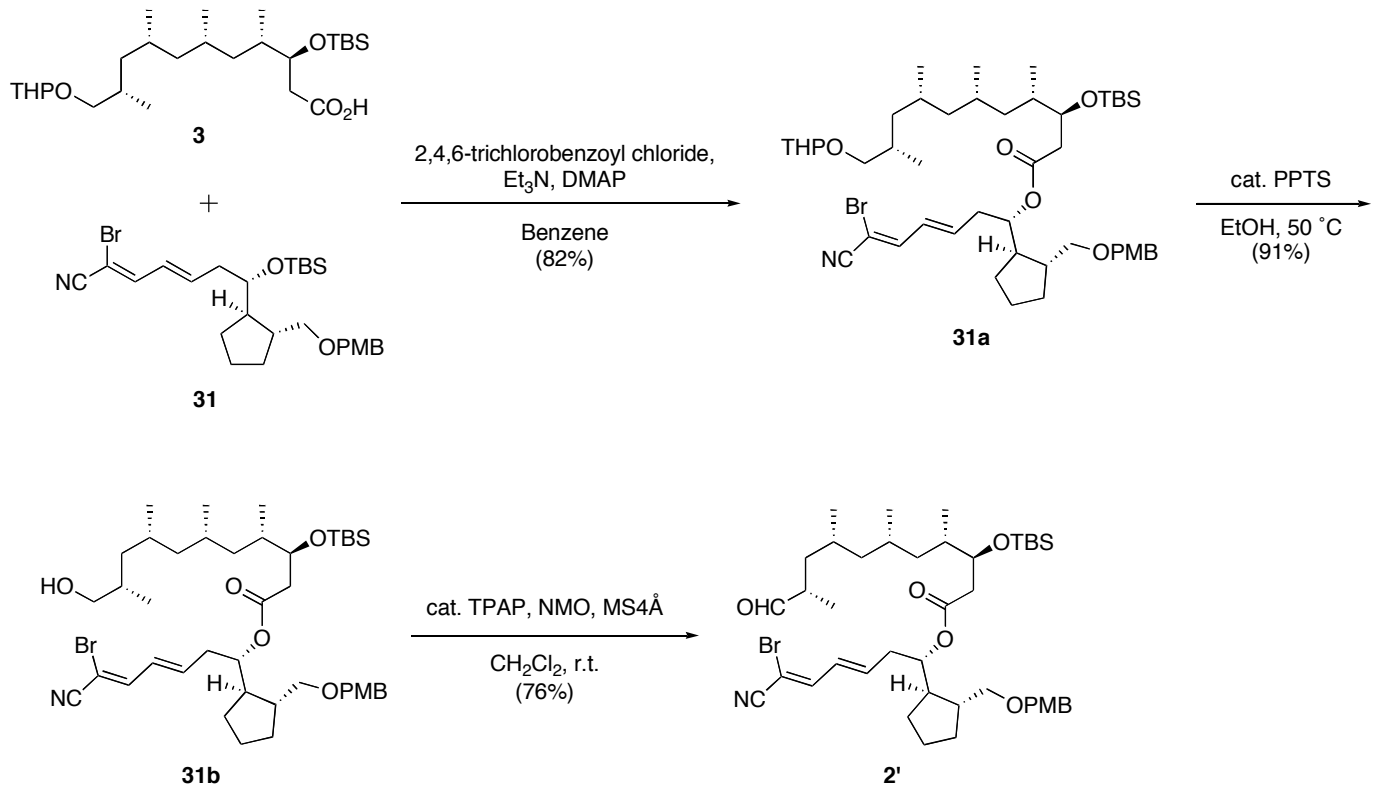

$(3 S, 4 S, 6 S, 8 R, 10 S)-((S, 3 E, 5 Z)-6-B r o m o-6-c y a n o-1-\{(1 R, 2 R)-2-[(4-$ methoxybenzyloxy)methyl]cyclopentyl $\}$ hexa-3,5-dienyl) 3-(tertbutyldimethylsilyloxy)-4,6,8,10-tetramethyl-11-(tetrahydro- $2 \mathrm{H}$-pyran-2yloxy)undecanoate (31a).
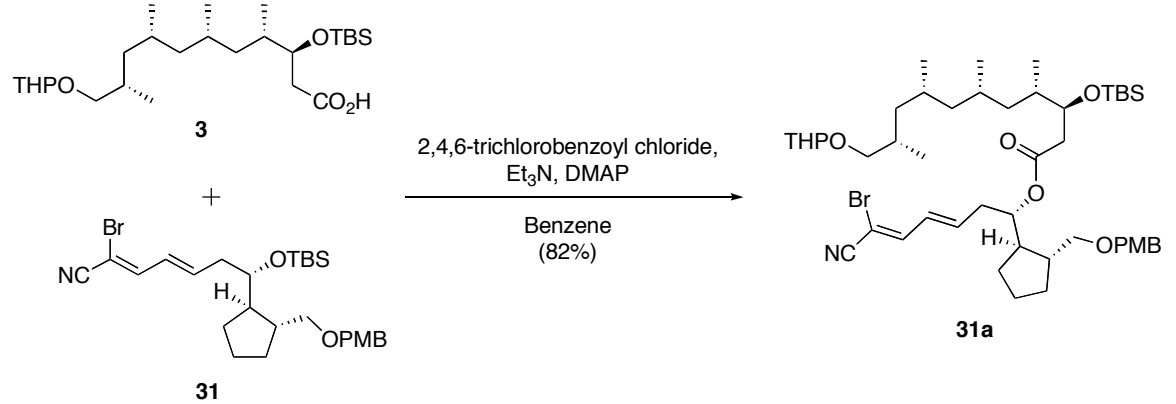

To a stirred solution of $\mathbf{3}(102 \mathrm{mg}, 0.216 \mathrm{mmol})$ in benzene $(3.80 \mathrm{ml})$ was added triethylamine $(60 \mu \mathrm{l}, 0.432 \mathrm{mmol})$ followed by $2,4,6$-trichlorobenzoyl chloride $(37 \mu \mathrm{l}$, $0.238 \mathrm{mmol})$ at room temperature. The resulting solution was stirred for $1 \mathrm{hr}$, then treated with a solution of $\mathbf{3 1}(113 \mathrm{mg}, 0.270 \mathrm{mmol})$ and DMAP $(34.0 \mathrm{mg}, 0.281 \mathrm{mmol})$ in benzene $(1.5 \mathrm{ml})$, and stirred for additional $30 \mathrm{~min}$. The reaction was quenched with saturated aqueous $\mathrm{NaHCO}_{3}$ solution and the aqueous phase was extracted with EtOAc. The combined organic extracts were dried over anhydrous $\mathrm{Na}_{2} \mathrm{SO}_{4}$ and concentrated in vacuo. Flash chromatography (25:1 hexanes/EtOAc) afforded 31a $(156 \mathrm{mg}, 82 \%)$ as a colorless oil. ${ }^{1} \mathrm{H}-\mathrm{NMR}\left(270 \mathrm{MHz}, \mathrm{CDCl}_{3}\right) \delta 0.03(\mathrm{~s}, 3 \mathrm{H}), 0.07$ (s, 3H), 0.80-0.92 (m, 23H), 0.99-1.42 (m, 5H), 1.46-1.94 (m, 16H), 2.07 (m, 1H), 2.31-2.60 (m, 4H), 3.10$3.39(\mathrm{~m}, 3 \mathrm{H}), 3.45-3.59(\mathrm{~m}, 2 \mathrm{H}), 3.80(\mathrm{~s}, 3 \mathrm{H}), 3.86(\mathrm{~m}, 1 \mathrm{H}), 4.06(\mathrm{~m}, 1 \mathrm{H}), 4.41(\mathrm{~d}, 1 \mathrm{H}$, $J=11.9 \mathrm{~Hz}), 4.44(\mathrm{~d}, 1 \mathrm{H}, J=11.9 \mathrm{~Hz}), 4.57(\mathrm{~m}, 1 \mathrm{H}), 4.96(\mathrm{~m}, 1 \mathrm{H}), 6.24(\mathrm{~m}, 1 \mathrm{H}), 6.38$ $(\mathrm{dd}, 1 \mathrm{H}, J=15.5,9.9 \mathrm{~Hz}), 6.87(\mathrm{~d}, 2 \mathrm{H}, J=8.6 \mathrm{~Hz}), 7.11(\mathrm{~d}, 1 \mathrm{H}, J=9.9 \mathrm{~Hz}), 7.24(\mathrm{~d}$, $2 \mathrm{H}, J=8.6 \mathrm{~Hz}) ;{ }^{13} \mathrm{C}-\mathrm{NMR}\left(67.5 \mathrm{MHz}, \mathrm{CDCl}_{3}\right) \delta-4.7,-4.4,14.5,16.4,16.5,18.0,19.4$, $19.5,20.4,20.6,25.0,25.5,25.8,26.9,27.0,27.2,29.6,30.1,30.6,30.8,30.9,35.4$, 36.6, 39.7, 39.9, 40.8, 40.9, 41.4, 45.0, 45.8, 55.1, 61.9, 62.1, 71.6, 72.7, 73.7, 73.9, 
74.0, 75.6, 86.6, 98.6, 98.9, 113.6, 116.5, 128.2, 128.9, 130.5, 143.6, 145.2, 159.0, 171.5; HRMS (FAB, $m$-NBA) $[\mathrm{M}+\mathrm{Na}]^{+}$calcd for $\mathrm{C}_{47} \mathrm{H}_{76} \mathrm{O}_{7} \mathrm{BrSiNa} 896.4472$, found 896.4467.

$(3 S, 4 S, 6 S, 8 R, 10 S)-((S, 3 E, 5 Z)-6-B r o m o-6-c y a n o-1-\{(1 R, 2 R)-2-[(4-$ methoxybenzyloxy)methyl] cyclopentyl\}hexa-3,5-dienyl) 3-(tertbutyldimethylsilyloxy)-11-hydroxy-4,6,8,10-tetramethylundecanoate (31b)
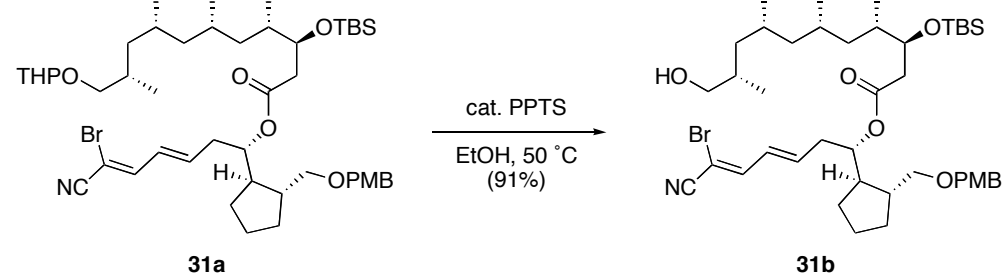

To a solution of $31 \mathrm{a}(156 \mathrm{mg}, 0.179 \mathrm{mmol})$ in EtOH $(5.0 \mathrm{ml})$ was added PPTS $(22.4$ $\mathrm{mg}, 89.4 \mu \mathrm{mol})$. The resulting solution was stirred at $50{ }^{\circ} \mathrm{C}$. After $12 \mathrm{hr}$, the reaction mixture was diluted with water and the aqueous phase was extracted with EtOAc. The combined organic extracts were dried over anhydrous $\mathrm{Na}_{2} \mathrm{SO}_{4}$ and concentrated in vacuo. Flash chromatography (7:1 hexanes/EtOAc) afforded 31b (129 mg, 91\%) as a colorless oil. ${ }^{1} \mathrm{H}-\mathrm{NMR}\left(270 \mathrm{MHz}, \mathrm{CDCl}_{3}\right) \delta 0.03(\mathrm{~s}, 3 \mathrm{H}), 0.07$ (s, 3H), 0.80-0.91 (m, 24H), 0.94-1.41 (m, 4H), 1.45-1.81 (m, 9H), $1.88(\mathrm{~m}, 1 \mathrm{H}), 2.06(\mathrm{~m}, 1 \mathrm{H}), 2.31-2.60(\mathrm{~m}$, 4H), 3.24-3.47 (m, 4H), $3.80(\mathrm{~s}, 3 \mathrm{H}), 4.06(\mathrm{~m}, 1 \mathrm{H}), 4.37$ (d, 1H, J = 11.9 Hz), 4.41 (d, $1 \mathrm{H}, J=11.9 \mathrm{~Hz}), 4.93(\mathrm{~m}, 1 \mathrm{H}), 6.21(\mathrm{~m}, 1 \mathrm{H}), 6.34(\mathrm{dd}, 1 \mathrm{H}, J=15.2,9.9 \mathrm{~Hz}), 6.83(\mathrm{~d}$, $2 \mathrm{H}, J=8.2 \mathrm{~Hz}), 7.08(\mathrm{~d}, 1 \mathrm{H}, J=9.9 \mathrm{~Hz}), 7.24(\mathrm{~d}, 2 \mathrm{H}, J=8.2 \mathrm{~Hz}) ;{ }^{13} \mathrm{C}-\mathrm{NMR}(67.5$ $\left.\mathrm{MHz}, \mathrm{CDCl}_{3}\right) \delta-4.7,-4.4,14.4,16.0,18.0,20.5,20.6,25.0,25.8,27.1,27.3,29.7$, 30.2 , 33.2, 35.5, 36.7, 39.5, 39.7, 41.0, 41.5, 45.0, 45.7, 55.1, 69.1, 71.7, 72.7, 74.0, 75.6, 86.6, 113.7, 116.6, 128.3, 129.0, 130.5, 143.6, 145.3, 159.1, 171.6.

$(3 S, 4 S, 6 S, 8 R, 10 S)-((S, 3 E, 5 Z)-6-B r o m o-6-c y a n o-1-\{(1 R, 2 R)-2-[(4-$ methoxybenzy loxy) methyl] cyclopentyl\}hexa-3,5-dienyl)

3-(tertbutyldimethylsilyloxy)-4,6,8,10-tetramethyl-11-oxoundecanoate (2')
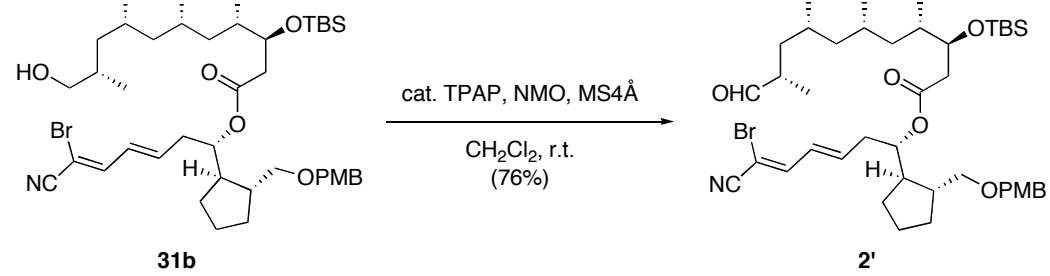

To a solution of $\mathbf{3 1 b}(42.1 \mathrm{mg}, 53.4 \mathrm{mmol})$ in $\mathrm{CH}_{2} \mathrm{Cl}_{2}(1.0 \mathrm{ml})$ were added dried MS4 $\AA$ (100 mg), TPAP (1.00 mg, $2.66 \mu \mathrm{mol})$ and NMO (12.5 mg, $0.106 \mathrm{mmol})$ at room temperature. The resulting solution was stirred for $30 \mathrm{~min}$ and filtered through a silica pad. After evaporation of the filtrate, the residue was purified by flash chromatography (15:1 hexanes/EtOAc) to afford 2' $(31.9 \mathrm{mg}, 76 \%)$ as a colorless oil. ${ }^{1} \mathrm{H}-\mathrm{NMR}(270$ $\left.\mathrm{MHz}_{1} \mathrm{CDCl}_{3}\right) \delta 0.03(\mathrm{~s}, 3 \mathrm{H}), 0.07(\mathrm{~s}, 3 \mathrm{H}), 0.80-0.91(\mathrm{~m}, 21 \mathrm{H}), 0.93-1.10(\mathrm{~m}, 2 \mathrm{H}), 1.07$ $(\mathrm{d}, 3 \mathrm{H}, J=6.9 \mathrm{~Hz}), 1.18-1.43(\mathrm{~m}, 2 \mathrm{H}), 1.47-1.76(\mathrm{~m}, 9 \mathrm{H}), 1.88(\mathrm{~m}, 1 \mathrm{H}), 2.06(\mathrm{~m}, 1 \mathrm{H})$, 2.30-2.59 (m, 4H), $3.31(\mathrm{~m}, 2 \mathrm{H}), 3.80(\mathrm{~s}, 3 \mathrm{H}), 4.06(\mathrm{~m}, 1 \mathrm{H}), 4.40(\mathrm{~d}, 1 \mathrm{H}, J=11.9 \mathrm{~Hz})$, $4.44(\mathrm{~d}, 1 \mathrm{H}, J=11.9 \mathrm{~Hz}), 4.96(\mathrm{~m}, 1 \mathrm{H}), 6.27(\mathrm{~m}, 1 \mathrm{H}), 6.38(\mathrm{dd}, 1 \mathrm{H}, J=15.6,9.9 \mathrm{~Hz})$, $6.87(\mathrm{~d}, 2 \mathrm{H}, J=8.6 \mathrm{~Hz}), 7.11(\mathrm{~d}, 1 \mathrm{H}, J=9.9 \mathrm{~Hz}), 7.24(\mathrm{~d}, 2 \mathrm{H}, J=8.6 \mathrm{~Hz}), 9.60(\mathrm{~s}, 1 \mathrm{H}$, 
$\mathrm{CHO}) ;{ }^{13} \mathrm{C}-\mathrm{NMR}\left(67.5 \mathrm{MHz}, \mathrm{CDCl}_{3}\right) \delta-4.7,-4.4,13.0,14.6,18.0,20.4,20.5,25.0$, $25.8,27.3,27.4,29.7,30.2,35.5,36.4,36.7,39.6,40.8,41.5,44.2,45.0,45.3,55.2$, 71.6, 72.7, 74.0, 75.6, 86.7, 113.7, 116.6, 128.3, 129.0, 130.5, 143.7, 145.3, 159.1, 171.6, 205.1. 